This item was submitted to Loughborough's Research Repository by the author.

Items in Figshare are protected by copyright, with all rights reserved, unless otherwise indicated.

\title{
Ito maps and analysis on path spaces
}

PLEASE CITE THE PUBLISHED VERSION

LICENCE

CC BY-NC-ND 4.0

\section{REPOSITORY RECORD}

Elworthy, K.D., and Xue-Mei Li. 2019. "Ito Maps and Analysis on Path Spaces". figshare. https://hdl.handle.net/2134/2440. 


\title{
Itô maps and analysis on path spaces
}

\author{
K. D. Elworthy \& Xue-Mei Li
}

\begin{abstract}
We consider versions of Malliavin calculus on path spaces of compact manifolds with diffusion measures, defining Gross-Sobolev spaces of differentiable functions and proving their intertwining with solution maps, $\mathcal{I}$, of certain stochastic differential equations. This is shown to shed light on fundamental uniqueness questions for this calculus including uniqueness of the closed derivative operator $d$ and Markov uniqueness of the associated Dirichlet form. A continuity result for the divergence operator by Kree and Kree is extended to this situation. The regularity of conditional expectations of smooth functionals of classical Wiener space, given $\mathcal{I}$, is considered and shown to have strong implications for these questions. A major role is played by the (possibly sub-Riemannian) connections induced by stochastic differential equations: Damped Markovian connections are used for the covariant derivatives.
\end{abstract}

\section{Introduction}

A natural approach to geometric analysis on path spaces, or loop spaces, of manifolds is to base it on continuous paths with Brownian motion (or other) diffusion) measure. It became clear in the 1970's from the early work of L. Gross on analysis on Banach spaces with Gaussian measures, that in such analysis the differentiation should be restricted to differentiation in directions given by a certain Hilbert space, the Cameron-Martin space. These $H$-derivatives formed the basis of the highly successful Malliavin Calculus, see e.g. Malliavin [36] [35]. Key tools in this were the Sobolev spaces they generated, see for example the books by Ikeda-Watanabe [30] and Nualart [38]. For paths on a Riemannian manifold, here based paths for simplicity, with Brownian motion measure, it was realized that the Cameron-Martin space should be replaced by Hilbert spaces of tangent vectors at almost all points of the path space: the so called Bismut tangent spaces. These are described in terms of parallel translation of the usual Cameron-Martin space of finite energy paths in the tangent space to $M$ at the base point, Jones-Léandre [31]. The parallel translation was that of the Levi-Civita connection. This was extended to more general connections by Driver in [9] whose work led to rapid progress in creating a Sobolev calculus (depending on the choice of connections) over the path spaces and loop spaces see e.g. Aida[1], Léandre [33]. When $M$ has curvature a major difficulty in this analysis comes from the nonholonomic nature of the Bismut tangent "bundle". There are no known "local charts" which adequately preserve the structure. The standard method, as in Driver [9] has

Key Words. Path space, Malliavin Calculus, Markov uniqueness, Sobolev spaces, weak derivatives, Markovian connection, Itô map, Banach manifold, divergence operator. 
been to use the stochastic development which gives a measure theoretic isomorphism of the Wiener space of based paths on Euclidean space with that on the manifold, and classically gives a diffeomorphism between the corresponding spaces of finite energy paths. However although this is smooth in the sense of Malliavin calculus, in general its derivative does not map the Cameron-Martin space to the Bismut Hilbert spaces. Moreover it seems clear from X-D Li [34] that the composition of it with a differentiable function, say in $\mathbb{D}^{p, 1}$, on the path space may not be differentiable on the flat space, e.g. not in $\mathbb{D}^{q, 1}$ for any $1 \leq q<\infty$ : a loss of differentiability occurs. There is an intertwining formula, Thm 2.6 in Cruzeiro-Malliavin [6], but it is for differentiation given by "tangent processes" not by tangent vectors.

There are also fundamental unresolved uniqueness problems in the calculus on these path spaces. The most basic is of the derivative operator itself: a standard approach is to take the closure in $L^{p}$ of the $H$-derivative defined on some initial domain of manifestly regular functions, e.g. smooth cylindrical functions or bounded Fréchet differentiable functions with bounded derivatives. In Wiener space the result does not depend on any, reasonable, choice of such initial domain, Sugita [45]. For paths on $M$ when there is non-zero curvature this is not known. Alhough there is a self-adjoint analogue $d^{*} d$ of the finite dimensional Laplace-Beltrami operator it is unknown whether it is essentially self-adjoint or whether it, or equivalently the associated Dirichlet form, has Markov uniqueness, taking the space of smooth cylindrical functions as initial domain. The latter concept relates to the uniqueness of a Markov process on the path space which would play the role of a Brownian motion (or Ornstein-Uhlenbeck process), see e.g. Eberle [12] and $\S 6$ below. Note that Aida has shown that such operators on certain finite co-dimensional submanifolds of Wiener space [3] are essentially self adjoint, and similarly for paths and loops on Lie groups [4]. An earlier work of Costa has shown the essential self-adjointness for a larger core on such path groups.

Here we continue the approach of Aida-Elworthy [2], Aida [1], Elworthy-LeJan-Li [17] and Elworthy-Li [19] using Itô maps, i.e. solution maps of stochastic differential equations, as substitutes for charts, and filtering techniques. We work with a fairly general class of, possibly degenerate, diffusion measures, with metric connections to define the Bismut tangent spaces. The stochastic differential equations are those whose solutions form the given diffusion process on $M$. We take $M$ compact and all coefficients smooth. The Itô maps are then infinitely differentiable in the sense of Malliavin Calculus, but as with the stochastic development their H-derivatives will not in general map into the Bismut tangent spaces, nor can we expect there to be a 'chain rule' to say that composition with them maps "differentiable" functions to 'differentiable' functions. However if we restrict to stochastic differential equations whose associated connection, in the sense of Elworthy-LeJan-Li [17] [15], agrees with the connection defining the Bismut tangent spaces, it turns out that such compositions are well behaved. The aim of this article is to describe this and its possible deficiences, and show what light it sheds on the fundamental uniqueness questions mentioned above. In particular we show that the latter are related to a question on Classical Wiener space concerning the regularity of differentiable functions after conditioning with respect to any of these Itô maps, see Remark 7.9 below.

Since we are working in greater generality than usual, the calculus on path space is developed from scratch, based on the integration by parts formulae of ElworthyLi [18] and Elworthy-LeJan-Li [15], [16]. This is done in section 4 for scalars and with a covariant calculus in section 8 more generally. In section 2 the basic setup of Bismut tangent bundles, damped parallel translations, stochastic differential equations and associated connections, are described. In section 3 there is the key result, Theorem 
3.4, that if the connection associated to the stochastic differential equation is the same as that used to define the Bismut tangent spaces then the Itô map can be used to pull back any measurable $\mathcal{H}$-1-form $\phi$ on the path space of $M$ to an $H$-1-form on flat Wiener space. We also give an explicit expression for the pull back $\mathcal{I}^{*} \phi$ as a stochastic integral. From this one obtains the pull back theorem, or chain rule, for functions in a Sobolev space $\mathbb{D}^{p, 1}\left(\mathcal{C}_{x_{0}} M ; \mathbf{R}\right)$, Theorem 4.2 and Corollary 4.3. In particular if $f \in \mathbb{D}^{p, 1}\left(\mathcal{C}_{x_{0}} M ; \mathbf{R}\right)$ then $f \circ \mathcal{I} \in \mathbb{D}^{p, 1}(\Omega ; \mathbf{R})$ and $d(f \circ \mathcal{I})=\mathcal{I}^{*}(d f)$ given some conditions on the connection.

In section 5 we consider the divergence operator acting on $\mathcal{H}$-vector fields and its intertwining by these Itô maps. We show that $V$ lies in its domain if a certain pull back of $V$ is in the domain of the divergence on flat space, Corollary 5.2. This enable us to extend the flat space result of Kree-Kree [32] and see that $\mathbb{D}^{p, 1} \mathcal{H}$-vector fields lie in the domain of the divergence, Theorem 5.8, a crucial result for our discussion of weak differentiability later.

In section 6 we introduce weak differentiability and the weak Sobolev spaces $W^{p, 1}$. Theorem 6.1 extends the chain rule to a precise intertwining:

$$
f \in W^{p, 1}\left(\mathcal{C}_{x_{0}} M ; \mathbf{R}\right) \text { iff } f \circ \mathcal{I} \in \mathbb{D}^{p, 1}\left(\mathcal{C}_{0} \mathbf{R}^{m} ; \mathbf{R}\right) .
$$

The question is posed as to whether $W^{p, 1}=\mathbb{D}^{p, 1}$, as in flat space. In Theorem 6.9, following Eberle [12], this is shown for the case of $p=2$ to be equivalent to Markov uniqueness, after demonstrating $W^{2,1}={ }^{0} W^{2,1}$, the latter being the weak Sobolev space used in [12]. A key step in the proof is to show that smooth cylindrical forms are dense in the space of $\mathbb{D}^{2,1} \mathcal{H}$-1-forms, Proposition 6.14.

Other uniqueness questions are considered in section 7.1. In particular it is shown that the closure of the differentiation operator is independent of the initial domain if that domain contains Cyl, the set of smooth cylindrical functions and consists of $B C^{2}$, twice Fréchet differentiable functions whose derivatives are bounded, Corollary 7.5. However we are not able to prove the uniqueness of $d$ when its initial domain is allowed to contain general $B C^{1}$ functions. Some of these results for the special case of Brownian motion measures and Levi-Civita connections are summarised in [21] correcting [25].

In the case when it is possible to find a stochastic differential equation whose Itô map has no redundant noise, all the main results in this article hold without the Condition $\left(M_{0}\right)$ which was often needed in the general situation. See section 9 . In particular we have $\mathcal{I}^{*} d=d \mathcal{I}^{*}$ on $L^{p}$ for $1<p<\infty$ and the Markov uniqueness. This applies to paths on Lie groups with left or right invariant connections and to paths on the orthonormal frame bundle of a Riemannian manifold with measure associated to the horizontal Laplacian. In this case our Itô map is essentially the stochastic development map and our results are an extension of some of the isomorphism results by Fang-Franchi [27] for path spaces on Lie groups.

The culmination of section 8 is Theorem 8.14 on the pull back by composition with $\mathcal{I}$ of higher order Sobolev spaces $\mathbb{D}^{q, k}$, and weak Sobolev spaces $W^{q, k}, k=$ $1,2 \ldots$ As for $k=1$, in the weak case there is a precise intertwining. To differentiate these Sobolev spaces requires a connection on the Bismut tangent 'bundle'. We use the 'damped Markovian' connection. This was introduced in Cruzeiro-Fang [5] for Brownian motion measures with Levi-Civita connections. One key point in this work is how well they fit into this situation, in some sense being induced by the derivative of $\mathcal{I}$, Proposition 8.2.

Although we work in considerably greater generality, the main results here, Theorem 3.4 and Theorem 8.14 on intertwining, Theorem 8.12 on the continuity of the 
divergence, Theorem 6.9 on Markov uniqueness, and Corollary 7.5 ( $B C^{2}$ functions are in $\mathbb{D}^{2,1}$ ), are essentially novel for the more standard case of Brownian motion measures and Levi-Civita connections (thought there is a version of Theorem 3.4 in Elworthy-Li [19]), as are the treatment of the covariant calculus in section 8 , and the importance shown for the rather general problem of the smoothness of conditional expectations in classical Wiener space, Remark 7.9.

\section{Contents}

1 Introduction

2 Basic Assumptions

3 Pull backs of $H$-forms by Itô maps.

4 Sobolev calculus on $\mathcal{C}_{x_{0}} M$ and its intertwining by Itô maps

5 The divergence operator and the spaces $\mathbb{D}^{p, 1} \mathcal{H}$

6 On the Markov Uniqueness of $d$

7 On uniqueness of $d$

8 Covariant Differentiation

9 Special case: no redundant noise

10 Appendix: The conditional expectations of exponential martingales

\section{Basic Assumptions}

Let $M$ be a $C^{\infty}$ connected manifold of dimension $n$. For simplicity assume it is compact. Otherwise some bounded geometry assumptions on the manifold and bounds on the coefficients of the stochastic differential equations we consider will need to be imposed. Let $\mathcal{A}$ be a smooth semi-elliptic second order operator with no zero order term. Assume its symbol $\sigma^{\mathcal{A}}: T^{*} M \rightarrow T M$ has constant rank $p$ so that its image is a sub-bundle $E$ of $T M$. It has a natural Riemannian metric induced by $\sigma^{\mathcal{A}}$. Let $\nabla$ be a metric connection on $E$. Then $\mathcal{A}$ can be written in the following form:

$$
\mathcal{A} f=\frac{1}{2} \operatorname{trace}_{E} \nabla_{-}\left(\left.d f\right|_{E}\right)+L_{A}(f)
$$

where $A$ is a smooth vector field. Denote by $\mu_{x_{0}}$ the law of the Markov process $\left(x_{t}: 0 \leq t \leq T\right)$ corresponding to $\mathcal{A}$ with initial value $x_{0}$ for some point $x_{0} \in M$ and fixed $T>0$.

Consider

$$
\mathcal{C}_{x_{0}} M=\left\{\sigma:[0, T] \rightarrow M \mid \sigma\left(x_{0}\right)=0, \sigma \text { is } C^{0}\right\},
$$

the space of continuous paths on $M$ starting from $x_{0}$ equipped with the probability measure $\mu_{x_{0}}$. 


\subsection{An SDE which induces the connection $\nabla$}

The underlying probability space $\Omega$ will be taken to be the canonical space $\mathcal{C}_{0} \mathbf{R}^{m}$, given by

$$
\Omega=\mathcal{C}_{0} \mathbf{R}^{m}=\left\{\omega:[0, T] \rightarrow \mathbf{R}^{m} \mid \omega(0)=0, \omega \text { is continuous }\right\},
$$

some natural number $m$. It is equipped with the Wiener measure $\mathbf{P}$ and its natural filtration $\left\{\mathcal{F}_{*}\right\}$. Let $\left\{B_{t}: 0 \leq t \leq T\right\}$ be the canonical Brownian motion on $\mathbf{R}^{m}$, that is $B_{t}(\omega)=\omega(t)$, the evaluation map.

Denote by $\mathbb{L}(E ; F)$ the space of bounded linear maps between linear spaces $E$ and $F$ and let $X: \mathbf{R}^{m} \times M \rightarrow T M$ be $C^{\infty}$ with $X(x) \in \mathbb{L}\left(\mathbf{R}^{m} ; T_{x} M\right)$ for each $x$. For each $x \in M$, Image $[X(x)]$ inherits an inner product. We shall choose $X$ so that Image $[X(x)]=E_{x}$ as a Hilbert space. Let $C^{r} \Gamma E$ be the space of $C^{r}$ sections of $E$. For $e$ in $\mathbf{R}^{m}$ let $X^{e}$ be the section of $E$ given by $X^{e}(x)=X(x)(e)$ and $Y: E \rightarrow \mathbf{R}^{m}$ the adjoint of $X$. Note that $X(x) Y(x)(v)=v$ for all $v \in T_{x} M$. Write $\operatorname{ker} X(x)$ and $[\operatorname{ker} X(x)]^{\perp}$ respectively for the kernel of the map $X(x)$ and its orthogonal complement. The result on which, Elworthy-LeJan-Li [15], and this article are based is the following, c.f. Quillen [40], Narasimhan-Ramanan [37]:

Proposition 2.1 (Elworthy-LeJan-Li [17], [15], c.f. Quillen[40]) For each such map $X: \mathbf{R}^{m} \times M \rightarrow T M$ there is a unique connection $\breve{\nabla}$ on $E$ such that

$$
\breve{\nabla}_{v} X^{e}=0, \quad \forall v \in T_{y} M, y \in M, e \in[\operatorname{ker} X(y)]^{\perp}
$$

This connection is metric. In fact

$$
\breve{\nabla}_{v} U=X(x) d(Y(U(\cdot)))(v), \quad v \in T_{x} M, U \in C^{1} \Gamma E .
$$

Furthermore all metric connections on $E$ can be obtained this way for some $X$ and some number $m$.

- Assumption $(X)$. By this proposition we can and will suppose from now on that map $X$ induces the Riemannian metric and the connection $\nabla$ on $E$. Hence (2.3) holds for $\nabla$.

For $A$ as in (2.1), consider the stochastic differential equation

$$
d x_{t}=X\left(x_{t}\right) \circ d B_{t}+A\left(x_{t}\right) d t, \quad 0 \leq t \leq T .
$$

It induces the diffusion measure $\mu_{x_{0}}$ on $M$.

\subsubsection{Examples [15]}

Example 1 (Gradient S.D.E.). Consider a Riemannian manifold $M$ isometrically immersed in $\mathbf{R}^{m}$ with immersion $j$. Set $X^{e} \equiv X(\cdot)(e)=\nabla\langle j(\cdot), e\rangle$. Then $X(x): \mathbf{R}^{m} \rightarrow$ $T M$ is the orthogonal projection of $\mathbf{R}^{m}$ to $T_{x} M$. The stochastic differential equation has solutions which are Brownian motions on $M$ and it induces the Levi-Civita connection on $M$ as its associated connection.

Example 2. (Left invariant S.D.E.). Let $M$ be a Lie group with left invariant metric, identity $e$ and Lie algebra $\mathfrak{g}:=T_{e} G$. Let $\left(B_{t}\right)$ be a Brownian motion on $\mathfrak{g}$. The connection associated to the left invariant stochastic differential equation

$$
d x_{t}=X\left(x_{t}\right) \circ d B_{t}
$$


is the flat left invariant connection. Here $X(e): \mathbf{R}^{n} \rightarrow \mathcal{G}$ is some isometry and $X(g) e=\left(T L_{g}\right) X(e)$. The solution of the equation is a process on the Lie group whose filtration is the same as that of the noise $\left(B_{t}\right)$. It is a Brownian motion if the metric is bi-invariant.

Example 3 (Symmetric space S.D.E.). Let $M=G$ be a Lie group with bi-invariant metric. It has a standard symmetric space structure:

$$
G=\frac{G \times G}{\{(g, g): g \in G\}}
$$

where $G \times G$ acts on $G$ as follows:

$$
\left(g_{1}, g_{2}\right) x=g_{1} x g_{2}^{-1} .
$$

Denote by $L_{x}$ and $R_{x}$ respectively the left and right group multiplications. Consider the stochastic differential equation on $G$

$$
d x_{t}=\frac{1}{\sqrt{2}} T L_{x_{t}} \circ d B_{t}-\frac{1}{\sqrt{2}} T R_{x_{t}} \circ d B_{t}^{\prime}
$$

where $\left(B_{t}\right)$ and $\left(B_{t}^{\prime}\right)$ are two independent Brownian motions on $\mathfrak{g}$. The corresponding connection is the Levi-Civita connection on $G$.

Example 4 (Canonical SDE on frame bundles). Let $N$ be an $m$ dimensional Riemannian manifold and let $M$ be its orthonormal frame bundle, $M=O N$, with $\pi$ : $O N \rightarrow N$ the projection. Using the Levi-Civita connection for $N$ consider the canonical stochastic differential equation on $O N$. Then $X(u) e=H_{u}(u(e))$ where $H_{u}$ : $T_{\pi(u)} N \rightarrow T_{u} O N$ denotes the horizontal lift map. Then $E$ is the horizontal tangent bundle of $O N$ and $p=m=\operatorname{dim} N$. The connection on $E$ is the flat connection induced by the trivialization $X$. The solutions to the S.D.E. on $O N$ project to Brownian motions on $N$, and are the horizontal lifts of those Brownian motions.

\subsection{The Covariant Differentiation operator $\frac{\mathbb{D}}{d t}$}

There is an adjoint semi-connection, $\nabla^{\prime}$, of $\nabla$. For each smooth vector field $V$ on $M$ this gives a derivative

$$
\nabla_{u}^{\prime} V \in T_{y} M
$$

for each $u \in E_{y}, y \in M$. It is defined by

$$
\nabla_{u}^{\prime} V=\nabla_{v} U+[U, V](y)
$$

for $v=V(y)$ and $U$ any smooth section of $E$ with $U(y)=u$.

Using $\nabla$ there are parallel translations along smooth paths $\sigma$ in $M$

$$
/ / t=/ / t(\sigma): E_{\sigma(0)} \rightarrow E_{\sigma(t)}
$$

and these preserve the inner products. Using $\nabla^{\prime}$ we obtain parallel translation

$$
\|_{t}^{\prime}=/_{t}^{\prime}(\sigma): T_{\sigma(0)} M \rightarrow T_{\sigma(t)} M
$$

along smooth paths which are 'horizontal'. (A path $\sigma$ is horizontal if $\dot{\sigma}(t)$ belongs to $E_{\sigma(t)}$ for each $t$.) There are also the operators $\frac{D}{d t}$ and $\frac{D^{\prime}}{d t}$

$$
\frac{D}{d t} U_{t}=/ / t \frac{d}{d t}\left(/ / t^{-1} U_{t}\right) \in E_{\sigma(t)}
$$




$$
\frac{D^{\prime}}{d t} V_{t}=/ /_{t}^{\prime} \frac{d}{d t}\left(/ /_{t}^{\prime-1} V_{t}\right) \in T_{\sigma(t)} M
$$

defined for vector fields along $\sigma$, for $U_{t} \in E_{\sigma(t)}$ each $t$, and for $\sigma$ horizontal in the case of $\frac{D^{\prime}}{d t}$.

We will need 'damped' versions of these operations. Let $Z$ be a vector field on $M$. When $Z(x) \in E_{x}$ for each $x$, the damped parallel translation $W_{t}^{Z} \equiv W_{t}^{Z}(\sigma)$ : $T_{\sigma(0)} M \rightarrow T_{\sigma(t)} M$ along a horizontal smooth path $\sigma$ is defined by

$$
\begin{cases}\frac{D^{\prime}}{d t}\left[W_{t}^{Z}\left(v_{0}\right)\right] & =-\frac{1}{2} \operatorname{Ric}^{\#}\left(W_{t}^{Z}\left(v_{0}\right)\right)+\nabla_{W_{t}^{Z}\left(v_{0}\right)} Z, \quad 0 \leq t \leq T \\ W_{0}^{Z}\left(v_{0}\right) & =v_{0} .\end{cases}
$$

Here Ric ${ }^{\#}: T M \rightarrow E$ is defined by the Ricci curvature Ric corresponding to the connection $\nabla:\left\langle\operatorname{Ric}^{\#}(u), v\right\rangle_{y}=\operatorname{Ric}_{y}(u, v)=\operatorname{trace}_{E}\langle R(u,-)-, v\rangle_{y}$. Under these conditions the corresponding operator $\frac{\mathbb{D}}{d t}$ on vector fields along $\sigma$ is given by

$$
\frac{\mathbb{D}}{d t} V_{t}=W_{t}^{Z} \frac{d}{d t}\left(\left(W_{t}^{Z}\right)^{-1} V_{t}\right)
$$

Thus

$$
\frac{\mathbb{D}}{d t}=\frac{D^{\prime}}{d t}+\frac{1}{2} \operatorname{Ric}^{\#}-\nabla Z
$$

In this case the damped (and undamped) parallel translation is defined almost surely along the sample paths of the solutions to our stochastic differential equation (2.5), with $\frac{D^{\prime}}{d t}$ and $\frac{\mathbb{D}}{d t}$ being defined correspondingly on suitable vector fields along the paths.

If $Z$ is not a section of $E$ then the solution paths are not 'horizontal' and it is convenient to introduce an auxiliary connection $\nabla^{1}$ on $T M$. To obtain this take a Riemannian metric on $T M$. Let $E^{\perp}$ be the orthogonal bundle to $E$ in $T M$ and take any metric connection $\nabla^{\perp}$ on $E^{\perp}$. Set

$$
\nabla^{1}=\nabla+\nabla^{\perp}
$$

and let $\nabla^{1^{\prime}}$ be its adjoint. Now extend the definition of $\frac{\mathbb{D}}{d t}$ to define

$$
\frac{\mathbb{D}}{d t} V_{t}:=\frac{D^{1^{\prime}}}{d t} V_{t}+\frac{1}{2} \operatorname{Ric}^{\#} V_{t}-\nabla_{V_{t}}^{1} Z
$$

for any suitably regular vector field $V$ along the paths of any continuous semi-martingale on $M$.

In particular $\frac{\mathbb{D}}{d t}$ is defined $\mu_{x_{0}}$-almost surely for suitably regular vector fields along the elements of $\mathcal{C}_{x_{0}} M$. It follows from Proposition 3.3.9 of Elworthy-LeJan-Li [15] and the Girsanov-Maruyama theorem that as such it depends only on $\nabla$ and $Z$ and not on the choice of $E^{\perp}$ or $\nabla^{\perp}$ provided that $Z-A \in \Gamma(E)$ where $A$ is the drift coefficient of the SDE. Consequently for such $Z$ the solution to

$$
\frac{\mathrm{D}}{d t} v_{t}=0
$$

given $v_{0} \in T_{x_{0}} M$ is defined along $\mu_{x_{0}}$-almost all paths and is independent of the choice of the auxiliary connection on $E^{\perp}$. When $Z=A$ it shall be denoted $W_{t}\left(v_{0}\right)$ to extend that defined by (2.7). With these extensions it remains true that

$$
\frac{\mathbb{D}}{d t} v_{t}=W_{t} \frac{d}{d t}\left(W_{t}^{-1}\left(v_{0}\right)\right)
$$




\subsubsection{Condition $\left(M_{0}\right)$}

Some additional conditions will sometimes be imposed on our connection $\nabla$ and on the stochastic differential equation. We are given a Riemannian metric only on $E$ and so will formulate the conditions in terms of that metric, avoiding using the metric we imposed on $T M$, even though for a compact manifold $M$ that is not really essential.

- Condition $\left(M_{0}\right)$ : The damped parallel translation $W_{t}$ satisfies:

(i) $\sup _{0 \leq t \leq T}\left|\left(\left.W_{t}^{-1}\right|_{E_{x_{t}}}\right)\right|_{\mathbb{L}\left(E_{x_{t}} ; T_{x_{0}} M\right)} \in L^{\infty} \quad$ and

(ii) $\sup _{0 \leq t \leq T}\left|\nabla_{W_{t}(-)} X\right|_{\mathbb{L}\left(T_{x_{0}} M ; \mathbb{L}\left(\mathbf{R}^{m} ; E_{x_{t}}\right)\right)} \in L^{\infty}$

- Condition $(M)$ : The adjoint connection $\nabla^{\prime}$ is metric for some Riemannian metric on $T M$, (which we will denote by $\langle\cdot, \cdot\rangle^{\prime}$ ).

Note that if $E=T M$ condition (M) holds with $\langle\cdot, \cdot\rangle^{\prime}=\langle\cdot, \cdot\rangle$ if and only if $\nabla$ is torsion skew symmetric as described by Driver in [9]. In particular Condition $(M)$ holds for the SDE's in Examples 1-3, section 2.1.1. For examples where it does not hold see Elworthy-LeJan-Li [15], in which there is also the following result (Proposition 3.3.11, p72):

Proposition 2.2 [15] For compact $M$,

$$
\sup _{0 \leq s \leq T}\left|W_{s}\right|_{\mathbb{L}\left(T_{x_{0}} M ; T_{x_{s}} M\right)} \text { and } \sup _{0 \leq s \leq T}\left|W_{s}^{-1}\right|_{\mathbb{L}\left(T_{x_{s}} M ; T_{x_{0}} M\right)}
$$

lie in $L^{p}$ for all $1 \leq p<\infty$. If also condition $(M)$ holds then both are in $L^{\infty}$. (Here we are using any Riemannian metric on $M$.)

From this we see immediately that condition $(M)$ implies condition $\left(M_{0}\right)$ in the compact case under consideration.

2.3 The $L^{2}$ tangent bundles $L^{2} \mathcal{E}, L^{2} T \mathcal{C}_{x_{0}} M$ and the Bismut tangent bundle $\mathcal{H}$

Recall that $\mathcal{C}_{x_{0}} M$ is a $C^{\infty}$ Banach manifold, Eells [13], see Eliasson [14], and its tangent space $T_{\sigma} \mathcal{C}_{x_{0}} M$ at a path $\sigma$ can be identified with the following space of vector fields along it:

$$
T_{\sigma} \mathcal{C}_{x_{0}} M=\left\{v:[0, T] \rightarrow T M \mid v_{t} \in T_{\sigma(t)} M, v_{0}=0\right\}
$$

By the $L^{2}$ tangent space $L^{2} \mathcal{E}_{\sigma}$ at $\sigma$ we mean the following set of measurable vector fields along $\sigma$ :

$$
L^{2} \mathcal{E}_{\sigma}=\left\{v:\left.[0, T] \rightarrow E\left|v_{t} \in E_{\sigma(t)},\right| v \cdot\right|_{L^{2} \mathcal{E}}<\infty\right\}
$$

where

$$
|v \cdot|_{L^{2} \mathcal{E}}:=\left(\int_{0}^{T}\left|v_{s}\right|^{2} d s\right)^{1 / 2} .
$$

These form the fibres of a smooth Hilbert bundle $L^{2} \mathcal{E}$ over $\mathcal{C}_{x_{0}} M$. It is associated to the principal bundle

$$
C_{x_{0}} O E \rightarrow \mathcal{C}_{x_{0}} M
$$


where $\pi: O E \rightarrow M$ is the orthonormal frame bundle of $E$ and

$$
C_{x_{0}} O E=\left\{u:[0, T] \rightarrow O E \mid u(0) \in \pi^{-1}\left(x_{0}\right)\right\} .
$$

Given the choice of a Riemannian metric on $T M$ extending that of $E$ we also have the Hilbert subbundle of $L^{2}$ tangent vectors $L^{2} T C_{x_{0}} M$, obtained as $L^{2} \mathcal{E}$ but using $T M$ rather than $E$. Then $L^{2} \mathcal{E}$ is a subbundle of $L^{2} T \mathcal{C}_{x_{0}} M$. Let

$$
\Pi: L^{2} T \mathcal{C}_{x_{0}} M \rightarrow L^{2} \mathcal{E}
$$

denote the orthogonal projection.

Using the metric connection $\nabla$ which we have imposed on $E$, we can define a family of subspaces $\mathcal{H}_{\sigma} \subset T_{\sigma} \mathcal{C}_{x_{0}} M$ :

$$
\mathcal{H}_{\sigma}:=\left\{\left.v \in T_{\sigma} \mathcal{C}_{x_{0}} M\left|\frac{\mathbb{D}}{d t} v_{t} \in E_{\sigma(t)}, \int_{0}^{T}\right| \frac{\mathbb{D} v_{t}}{d t}\right|_{\sigma(t)} ^{2} d t<\infty\right\}
$$

(with the usual convention of absolute continuity after translation back to $T_{x_{0}} M$ ). This is a Hilbert space under the obvious inner product

$$
\langle u, v\rangle_{\sigma}=\int_{0}^{T}\left\langle\frac{\mathbb{D} u_{t}}{d t}, \frac{\mathbb{D} v_{t}}{d t}\right\rangle_{\sigma(t)} d t .
$$

Note also that $\frac{\mathbb{D}}{d t}$ determines an isometry of $\mathcal{H}_{\sigma} \rightarrow L^{2} \mathcal{E}_{\sigma}$ for almost all $\sigma$, with inverse

$$
\text { W. : } L^{2} \mathcal{E}_{\sigma} \rightarrow \mathcal{H}_{\sigma}
$$

given by

$$
\mathbf{W}_{t}(v)=W_{t} \int_{0}^{t} W_{s}^{-1} v_{s} d s .
$$

Let $\mathcal{H}=\sqcup_{\sigma} \mathcal{H}_{\sigma}$. Then it inherits a vector bundle structure (over a subset of full measure in $\left.\mathcal{C}_{x_{0}} M\right)$ from $L^{2} \mathcal{E}$ via $\frac{\mathbf{D}}{d t}$ as does its dual $\mathcal{H}^{*}=\sqcup_{\sigma} \mathcal{H}_{\sigma}^{*}$.

In particular an $L^{p} H$-form (or written as $\mathcal{H}$-form) $\phi$ on $\mathcal{C}_{x_{0}} M$ is an $L^{p}$ section of $\mathcal{H}^{*}$, i.e. an assignment of $\phi_{\sigma}: \mathcal{H}_{\sigma} \rightarrow \mathbf{R}$, continuous linear, for almost all $\sigma$ in $\mathcal{C}_{x_{0}} M$, measurable in $\sigma$ in the sense that $\sigma \mapsto \phi_{\sigma}\left(\frac{\mathbb{D}}{d}-\right)$ is a measurable section of the dual of the vector bundle $L^{2} \mathcal{E}$ with

$$
\left.\left|\phi \|_{L^{p}}:=\int_{\mathcal{C}_{x_{0}} M}\right| \phi_{\sigma}\right|_{\mathcal{H}_{\sigma}^{*}} ^{p} d \mu_{x_{0}}(\sigma)<\infty .
$$

Let $L^{p} \Gamma \mathcal{H}^{*}$ be the space of equivalence classes of $L^{p} H$-forms.

Remark 2.3 Suppose $V \in L^{p} \Gamma \mathcal{H}$, the space of $L^{p} H$-vector fields on $\mathcal{C}_{x_{0}} M$. Then, for any inner product on $T_{x_{0}} M$ and almost all $\sigma \in \mathcal{C}_{x_{0}} M$

$$
\begin{aligned}
\sup _{t}\left|W_{t}^{-1} V_{t}(\sigma)\right| & =\sup _{t}\left|\int_{0}^{t} W_{s}^{-1} \frac{\mathbb{D}}{d s} V_{s}(\sigma) d s\right| \\
& \leq T^{\frac{1}{2}} \sup _{t}\left|W_{t}^{-1}\right|_{\mathbb{L}\left(E_{\sigma(t)} ; T_{x_{0}} M\right)}\|V\|_{\mathcal{H}_{\sigma}}
\end{aligned}
$$

and so $\sup _{t}\left|W_{t}^{-1} V_{t}(\sigma)\right|$ is in $L^{p}$ if condition $\left(M_{0}\right)$ holds. 


\section{Pull backs of $H$-forms by Itô maps.}

\subsection{The derivative $T \mathcal{I}$ of the Itô map and $\overline{T \mathcal{I}}$}

Let

$$
\begin{gathered}
\mathcal{I}: \mathcal{C}_{0} \mathbf{R}^{m} \rightarrow \mathcal{C}_{x_{0}} M \\
\mathcal{I}(\omega)_{t}=x_{t}(\omega)
\end{gathered}
$$

be the Itô map of (2.5) for $\left\{\xi_{t}: 0 \leq t \leq T\right\}$ the solution flow of (2.5) and $x_{t}(\omega)=$ $\xi_{t}\left(x_{0}, \omega\right)$. For each $\omega \in \mathcal{C}_{0} \mathbf{R}^{m}$, let

$$
T_{\omega} \mathcal{I}: H \equiv L_{0}^{2,1} \mathbf{R}^{m} \longrightarrow T_{x .(\omega)} \mathcal{C}_{x_{0}} M
$$

be its $H$-derivative in the sense of Malliavin calculus. Strictly speaking conventional Malliavin calculus just gives a derivative at each time $t$

$$
T_{\omega} \mathcal{I}_{t}: H \longrightarrow T_{x_{t}(\omega)} \mathcal{C}_{x_{0}} M
$$

However there is the formula, due to Bismut, for $v_{t}(\omega)=T_{\omega} \mathcal{I}_{t}(h), h \in H$ :

$$
v_{t}=T_{x_{0}} \xi_{t} \int_{0}^{t}\left(T_{x_{0}} \xi_{s}\right)^{-1} X\left(x_{s}\right) \dot{h}_{s} d s, \quad 0 \leq t \leq T
$$

where $T_{x_{0}} \xi_{t}: T_{x_{0}} M \rightarrow T_{x_{t}} M$ is the derivative at $x_{0}$ of $\xi_{t}$. This shows that we do have, for almost all $\omega \in \mathcal{C}_{0} \mathbf{R}^{m}$, a continuous linear version $T_{\omega} \mathcal{I}: H \rightarrow T_{x .(\omega)} \mathcal{C}_{x_{0}} M$. Moreover $\sup _{t}\left|T \mathcal{I}_{t}\right|_{\mathbb{L}\left(H ; T_{x_{t}} M\right)}$ lies in $L^{p}$ for all $1 \leq p<\infty$, (c.f. Proposition 2.2 below), for any Riemannian metric on our compact manifold $M$.

One of the key points in our discussion will be the decomposition of the 'noise' $\left\{B_{t}: 0 \leq t \leq T\right\}$ into 'redundant' and 'relevant' parts

$$
d B_{t}=\tilde{/}_{t} d \tilde{B}_{t}+\tilde{/}_{t} d \beta_{t}
$$

as described in Elworthy-Yor [23] for gradient systems and Elworthy-LeJan-Li [17], [15] more generally. Here

(i) $\tilde{/}_{t}(\omega): \mathbf{R}^{m} \rightarrow \mathbf{R}^{m}$ is an orthogonal transformation of $\mathbf{R}^{m}$, mapping $\operatorname{ker} X\left(x_{0}\right)$ to $\operatorname{ker} X\left(x_{t}(\omega)\right)$, given by parallel translation along $\left\{x_{t}: 0 \leq t \leq T\right\}$ using a connection on the trivial $\mathbf{R}^{m}$-bundle over $M$, canonically determined by $X$.

(ii) $\tilde{B}_{t}:=\int_{0}^{t} \tilde{/}_{s}^{-1} K^{\perp}\left(x_{s}\right) d B_{s}$, for $K^{\perp}(x)$ the orthogonal projection of $\mathbf{R}^{m}$ onto $[\operatorname{ker}(X(x))]^{\perp}$; so $\left\{\tilde{B}_{t}: 0 \leq t \leq T\right\}$ is a Brownian motion on $\left[\operatorname{ker} X\left(x_{0}\right)\right]^{\perp}$. It has the same filtration as that of $\left\{x_{t}: 0 \leq t \leq T\right\}$.

(iii) $\beta_{t}:=\int_{0}^{t} \tilde{/}_{s}^{-1} K\left(x_{s}\right) d B_{s}$ with $K(x)=\mathbf{1}-K^{\perp}(x)$; so $\left\{\beta_{t}: 0 \leq t \leq T\right\}$ is an $\mathcal{F}_{*}$-Brownian motion on $\operatorname{ker} X\left(x_{0}\right)$, independent of $\left\{x_{s}: 0 \leq s \leq T\right\}$. From the point of the view of the solution $\left\{x_{t}: 0 \leq t<\infty\right\}$ it is the 'redundant noise'.

From Elworthy-LeJan-Li [16] or equation (4.16) p79 of [15], we have the covariant Itô equation for $v_{t} \equiv T \mathcal{I}_{t}(h)$ any $h \in H$, using the connection $\nabla^{1^{\prime}}$ on $T M$, defined via (2.9),

$$
D^{1^{\prime}} v_{t}=\nabla_{v_{t}} X\left(\tilde{/} t t d \beta_{t}\right)-\frac{1}{2} \operatorname{Ric}^{\#}\left(v_{t}\right) d t+\nabla_{v_{t}}^{1} A d t+X\left(x_{t}\right) \dot{h}_{t} d t
$$


which may be written, using notation (2.10),

$$
\mathbb{D} v_{t}=\nabla_{v_{t}} X\left(\tilde{/}_{t} d \beta_{t}\right)+X\left(x_{t}\right) \dot{h}_{t} d t
$$

The equation (3.3) comes from (3.2) and the defining property, (2.3), of the connection.

For almost all $\sigma \in C_{x_{0}} M$ and $h \in H$ define

$$
\overline{T \mathcal{I}}_{\sigma}(h)=\mathbf{E}\left\{T_{\omega} \mathcal{I}(h) \mid x .(\omega)=\sigma\right\} .
$$

From (3.4), as in [15], we obtain a key property

Property 3.1 ( Elworthy-Li [24][19]) Suppose the connection defined by the SDE (2.5) is the same as that defining $\mathcal{H}$. Then the map $\overline{T \mathcal{I}}_{\sigma}$ gives a projection

$$
\overline{T \mathcal{I}}_{\sigma}: H \rightarrow \mathcal{H}_{\sigma}
$$

for almost all $\sigma \in C_{x_{0}} M$. It is given by

$$
\overline{T \mathcal{I}}_{\sigma}(h)_{t}=W_{t} \int_{0}^{t} W_{s}^{-1} X\left(\sigma_{s}\right) \dot{h}_{s} d s
$$

with isometric right inverse $v \mapsto \int_{0}^{\cdot} Y_{\sigma(s)}\left(\frac{\mathbb{D}}{d s} v_{s}\right) d s$.

\subsection{Some useful lemmas}

Lemma 3.1 Let $\left(M_{t}, 0 \leq t \leq T\right)$ be a continuous local martingale with respect to some filtration $\mathcal{G}_{*}$, with values in $\mathbb{L}\left(\mathbf{R}^{k} ; G\right)$ for some separable Hilbert space $G$. Suppose the tensor quadratic variation of $\left(M_{t}\right)$ has a continuous density with respect to $t$. Then the map

$$
f \mapsto \int_{0}^{T} d M_{s}\left(f_{s}\right) .
$$

is continuous in probability as a map

$$
L^{0}\left(\Omega, \mathcal{G}_{0}, P ; \mathbb{L}^{2}\left([0, T] ; \mathbf{R}^{k}\right)\right) \longrightarrow L^{0}\left(\Omega, \mathcal{G}_{T}, P ; G\right)
$$

Proof Suppose $\left\{f_{n}, n \geq 1\right\}$ is a sequence of $\mathcal{G}_{0}$ measurable functions converging in probability to $f$. For $m=1,2,3, \ldots$, set $\tau_{m}=\inf _{t>0}\left\{\sup _{n}\left\{\int_{0}^{t}\left|f_{n}(s)\right|^{2} d s\right\} \geq m\right\}$, giving it the value $T$ if the set is empty. Note that by going to a subsequence which converges almost surely we can assume that $\sup _{n}\left\{\int_{0}^{T}\left|f_{n}(s)\right|^{2} d s\right\}$ is almost surely finite and so these times increase to $T$ almost surely. They are also $\mathcal{G}_{0}$-measurable and so can be used as stopping times. The processes $\left\{\chi_{\left[0, \tau_{m}\right)}(\cdot) f_{n}\right\}_{n=1}^{\infty}$ are bounded and so converge in $L^{p}$ to $\chi_{\left[0, \tau_{m}\right)}(\cdot) f$ for each $m$ and $p<\infty$. Their stochastic integrals, after localisation, will then converge in $L^{p}$ and the result follows.

The next proposition extends the main technical tool used in Aida-Elworthy [2]. Compactness of $M$ is not used though non-explosion of the underlying diffusion needs to be assumed. First we record an easy consequence of the Burkholder-Davis-Gundy inequalities. 
Lemma 3.2 For $0<p<\infty$ let $c_{p}, C_{p}$ be the constants in the Burkholder-DavisGundy inequalities. If $\left\{Z_{t}: 0 \leq t \leq T\right\}$ is a real-valued continuous local martingale with respect to a filtration $\left\{\mathcal{G}_{t}: 0 \leq t \leq T\right\}$ with $Z_{0}=0$, then almost surely

$$
c_{p} \mathbf{E}\left\{\langle Z, Z\rangle_{T}^{\frac{p}{2}} \mid \mathcal{G}_{0}\right\} \leq \mathbf{E}\left\{\sup _{0 \leq t \leq T}\left|Z_{t}\right|^{p} \mid \mathcal{G}_{0}\right\} \leq C_{p} \mathbf{E}\left\{\langle Z, Z\rangle_{T}^{\frac{p}{2}} \mid \mathcal{G}_{0}\right\} .
$$

Proof Let $\lambda$ be non-negative, $\mathcal{G}_{0}$-measurable, and bounded. Then $\left\{\lambda^{\frac{1}{p}} Z_{t}: 0 \leq t \leq T\right\}$ is a $\mathcal{G}_{*}$-local martingale to which we can apply the Burkholder-Davis-Gundy inequalities to see

$$
c_{p} \mathbf{E} \lambda\langle Z, Z\rangle_{T}^{\frac{p}{2}} \leq \mathbf{E} \lambda \sup _{0 \leq t \leq T}\left|Z_{t}\right|^{p} \leq C_{p} \mathbf{E} \lambda\langle Z, Z\rangle_{T}^{\frac{p}{2}}
$$

giving the result.

$$
\text { Set } \mathcal{F}^{x_{0}}=\sigma\left\{x_{s}: 0 \leq s \leq T\right\} .
$$

Proposition 3.3 Assume condition $\left(M_{0}\right)$ holds. Then for all $1 \leq p<\infty$ there is a constant $\alpha_{p}$ with

$$
\mathbf{E}\left\{\sup _{0 \leq s \leq T}\left|W_{s}^{-1} T \mathcal{I}_{s}(h)\right|_{T_{x_{0}} M}^{p} \mid \mathcal{F}^{x_{0}}\right\} \leq \alpha_{p}\|h\|_{H}^{p}, \quad \text { all } h \in H \quad \text { a.s. }
$$

Proof Take $h \in H$. We only need to show the inequality for $p \geq 2$. From (3.4) we have the Itô equation for $u_{t}:=W_{t}^{-1}\left(T \mathcal{I}_{t}(h)\right)$ :

$$
d u_{t}=W_{t}^{-1} \nabla_{W_{t}\left(u_{t}\right)} X\left(\tilde{/} t d \beta_{t}\right)+W_{t}^{-1} X\left(x_{t}\right)\left(\dot{h}_{t}\right) d t
$$

giving

$$
\begin{aligned}
\sup _{0 \leq t \leq \tau}\left|u_{t}\right|^{p} & \leq 2^{p-1} \sup _{0 \leq t \leq \tau}\left|\int_{0}^{t} W_{s}^{-1} \nabla_{W_{s}\left(u_{s}\right)} X\left(\tilde{/}{ }_{s} d \beta_{s}\right)\right|^{p} \\
& +2^{p-1} \sup _{0 \leq t \leq \tau}\left|\int_{0}^{t} W_{s}^{-1} X\left(x_{s}\right)\left(\dot{h}_{s}\right) d s\right|^{p}
\end{aligned}
$$

for any $0 \leq \tau \leq T$. Set $\mathcal{G}_{t}=\mathcal{F}_{t} \vee \mathcal{F}^{x_{0}}$. Then $\left(\beta\right.$.) is a $\mathcal{G}_{*}$-Brownian motion and so we can apply Lemma 3.2 to give

$$
\begin{array}{r}
\mathbf{E}\left\{\sup _{0 \leq t \leq \tau}\left|\int_{0}^{t} W_{s}^{-1} \nabla_{W_{s}\left(u_{s}\right)} X\left(\tilde{I}_{s} d \beta_{s}\right)\right|^{p} \mid \mathcal{F}^{x_{0}}\right\} \\
\leq C_{p} \mathbf{E}\left\{\left(\int_{0}^{\tau} \sum_{j}\left|W_{s}^{-1} \nabla_{W_{s}\left(u_{s}\right)} X^{j}\right|^{2} d s\right)^{\frac{p}{2}} \mid \mathcal{F}^{x_{0}}\right\}
\end{array}
$$

for $X^{j}(x)=X(x)\left(e^{j}\right)$ where $e^{1}, \ldots, e^{m}$ is an orthonormal base for $\mathbf{R}^{m}$. Since $p \geq 2$, condition $\left(M_{0}\right)$ plus Jensen's inequality gives

$$
\mathbf{E}\left\{\left(\int_{0}^{\tau} \sum_{j}\left|W_{s}^{-1} \nabla_{W_{s}\left(u_{s}\right)} X^{j}\right|^{2} d s\right)^{\frac{p}{2}} \mid \mathcal{F}^{x_{0}}\right\}
$$




$$
\begin{aligned}
& \leq \text { const. }\left(\left.\left.\left|\sup _{0 \leq s \leq \tau}\right| W_{s}^{-1}\right|_{E_{x_{s}}}\right|_{\mathbb{L}\left(E_{x_{s}} ; T_{x_{0}} M\right)} \mid L^{\infty}\right)^{p} . \\
& \left(\left.\left.\left|\sup _{0 \leq s \leq \tau}\right| \nabla_{W_{s}(-)} X\right|_{\mathbb{L}\left(T_{x_{0}} M ; \mathbb{L}_{2}\left(R^{m} ; E_{x_{s}}\right)\right)}\right|_{L^{\infty}}\right)^{p}\left(\tau^{p / 2-1}\right) \mathbf{E}\left\{\int_{0}^{\tau}\left|u_{s}\right|^{p} d s \mid \mathcal{F}^{x_{0}}\right\} \\
& \leq \text { const. } \int_{0}^{\tau} \mathbf{E}\left\{\sup _{0 \leq r \leq s}\left|u_{r}\right|^{p} \mid \mathcal{F}^{x_{0}}\right\} d s .
\end{aligned}
$$

Applying condition $\left(M_{0}\right)$ to the second term on the right hand side of (3.7) we see that (3.7) leads to

$$
\mathbf{E}\left\{\sup _{0 \leq t \leq \tau}\left|u_{t}\right|^{p} \mid \mathcal{F}^{x_{0}}\right\} \leq \text { const. } \int_{0}^{\tau} \mathbf{E}\left\{\sup _{0 \leq r \leq s}\left|u_{r}\right|^{p} \mid \mathcal{F}^{x_{0}}\right\} d s+\text { const. }\left(\|h\|_{H}\right)^{p}
$$

and the result follows by Gronwall's lemma.

\subsection{The pull back map $\mathcal{I}^{*}$ and the push forward map $\overline{T \mathcal{I}(-)}$}

If $f: \mathcal{C}_{x_{0}} M \rightarrow \mathbf{R}$ is Fréchet $C^{1}$ with bounded derivative $d f: T \mathcal{C}_{x_{0}} M \rightarrow \mathbf{R}$ we see that $\mathcal{I}^{*}(d f)_{\omega}:=d f \circ T_{\omega} \mathcal{I}$ is almost surely defined as a continuous linear functional on $H$, (and by the usual approximation techniques this is $\left.\bar{d}(f \circ \mathcal{I})_{\omega}\right)$. Similarly we can pull back any geometric 1-form $\phi: T \mathcal{C}_{x_{0}} M \rightarrow \mathbf{R}$ to obtain an $H$-form $\mathcal{I}^{*}(\phi)=\phi(T \mathcal{I}-)$ on $\mathcal{C}_{0} \mathbf{R}^{m}$. If $f \in L^{2}\left(\mathcal{C}_{x_{0}} M ; \mathbf{R}\right)$ is an arbitrary element in $\operatorname{Dom}(\bar{d})$ we have now the $\mathcal{H}$-form $\bar{d} f_{\sigma}: \mathcal{H}_{\sigma} \rightarrow \mathbf{R}$ for almost all $\sigma \in \mathcal{C}_{x_{0}} M$. Set $v_{t}=T \mathcal{I}_{t}(h)$ for $h \in H$. From Bismut's formula (3.1) we cannot expect $v$. to be in $\mathcal{H}$ and thus the usual pull back map $\mathcal{I}^{*}: \Lambda^{1} \rightarrow L^{0}\left(\Omega ; H^{*}\right)$ defined by $\mathcal{I}^{*}(\phi)(h)=\phi(T \mathcal{I}(h))$, on geometric differential 1forms does not obviously extend to $\mathcal{H}$-forms. In particular it is not at all clear that we can define $\mathcal{I}^{*}(\bar{d} f)$. As shall be seen below we will need to interpret $\phi(v$. $)$ as a stochastic integral.

Theorem 3.4 Under the standing assumption Assumption $(X)$, the map $\phi \mapsto \mathcal{I}^{*} \phi:=$ $\phi \circ T \mathcal{I}$ defined on measurable geometric forms on $\mathcal{C}_{x_{0}} M$ extends to a continuous linear injective map

$$
\mathcal{I}^{*}: L^{0} \Gamma \mathcal{H}^{*} \quad \longrightarrow \quad L^{0}\left(\mathcal{C}_{0} \mathbf{R}^{m} ; H^{*}\right)
$$

from measurable $\mathcal{H}$-one forms on $\mathcal{C}_{x_{0}} M$ to measurable $H$-one-forms on $\mathcal{C}_{0} \mathbf{R}^{m}$, using the topology of convergence in probability. The map is given by the Ito stochastic integral

$$
\mathcal{I}^{*}(\phi)(h)=\int_{0}^{T}\left\langle\frac{\mathbb{D} \phi_{s}^{\#}}{d s}, \nabla_{T \mathcal{I}_{s}(h)} X\left(\tilde{/}_{s} d \beta_{s}\right)+X\left(x_{s}\right)\left(\dot{h}_{s}\right) d s\right\rangle_{x_{s}}, \quad h \in H
$$

using the filtration $\mathcal{G}_{t}:=\mathcal{F}_{t}^{\beta} \vee \mathcal{F}^{x_{0}}, 0 \leq t \leq T$. Moreover for $1 \leq p<\infty$,

(a) the map $\mathcal{I}^{*}$ restricts to a continuous linear map

$$
\mathcal{I}^{*}: L^{p+\varepsilon} \Gamma \mathcal{H}^{*} \longrightarrow L^{p}\left(\mathcal{C}_{0} \mathbf{R}^{m} ; H^{*}\right)
$$

for any $\varepsilon>0$. If condition $\left(M_{0}\right)$ holds, (3.9) holds for $\varepsilon=0$.

(b) if $\phi \in L^{0} \Gamma \mathcal{H}$

$$
\mathbf{E}\left\{\mathcal{I}^{*}(\phi(-)) \mid \mathcal{F}^{x_{0}}\right\}=\phi\left(\overline{T \mathcal{I}}_{x,}-\right)
$$

and

$$
\|\phi\|_{L^{p}} \leq\left\|\mathcal{I}^{*}(\phi)\right\|_{L^{p}}
$$


Consequently $\mathcal{I}^{*}\left[L^{0} \Gamma \mathcal{H}^{*}\right] \cap L^{p}\left(\mathcal{C}_{0} \mathbf{R}^{m} ; H\right)$ is closed in $L^{p}$ and is contained in $\mathcal{I}^{*}\left[L^{p} \Gamma \mathcal{H}^{*}\right]$ with equality if condition $\left(M_{0}\right)$ holds.

Proof (1) For $h \in H$ set $v_{t}=T \mathcal{I}_{t}(h)$. From (3.4),

$$
v_{t}=W_{t} \int_{0}^{t} W_{s}^{-1} \nabla_{v_{s}} X\left(\tilde{/}_{s} d \beta_{s}\right)+\mathbf{W}_{t}(X(x .)(\dot{h} .)) .
$$

Consider $\phi(v$.) for $\phi$ a geometric 1-form. We can treat $\phi$ as an $\mathcal{H}$-form by restriction. As such it has a dual $\mathcal{H}$-vector field $\phi^{\#}$, so if $u \in \mathcal{H}_{\sigma}$ then

$$
\phi(u)=\phi_{\sigma}(u)=\int_{0}^{T}\left\langle\frac{\mathbb{D}}{d t} u_{t}, \frac{\mathbb{D}}{d t} \phi_{t}^{\#}(\sigma)\right\rangle_{\sigma(t)} d t .
$$

Thus

$$
\phi(v .)=\phi\left(W_{t} \int_{0}^{t} W_{s}^{-1} \nabla_{v_{s}} X\left(\tilde{I}_{s} d \beta_{s}\right)\right)+\int_{0}^{T}\left\langle\frac{\mathbb{D}}{d t} \phi_{t}^{\#}, X\left(x_{t}\right) \dot{h}_{t}\right\rangle d t
$$

For the second term on the right hand side, we have

$$
\left|\int_{0}^{T}\left\langle\frac{\mathbb{D}}{d t} \phi_{t}^{\#}, X\left(x_{t}\right) \dot{h}_{t}\right\rangle d t\right| \leq\left\|\phi^{\#}\right\|_{\mathcal{H}_{x} .}\|h\|_{H}, \quad \text { a.s. }
$$

Consequently the map $h \mapsto \phi_{x}$. $\left(\mathbf{W} .(X(x).(\dot{h}\right.$. $))$ is in $H^{*}$ almost surely. For $p=0$ or $1 \leq p \leq \infty$ it gives an element of $L^{p}\left(\mathcal{C}_{0} \mathbf{R}^{m} ; H^{*}\right)$ depending continuously on the restriction of $\phi$ in $L^{p} \Gamma \mathcal{H}^{*}$.

(2) For the more interesting first term on the right hand side of (3.13),

$$
\Phi(h, \phi) \equiv \phi\left(W \cdot \int_{0}^{\cdot} W_{s}^{-1} \nabla_{v_{s}} X\left(/ / \tilde{l}_{s} d \beta_{s}\right)\right),
$$

we assume that $\phi$ is a geometric differential form on $\mathcal{C}_{x_{0}} M$ which extends to give linear functionals on the $L^{2}$ tangent spaces $L^{2} T_{\sigma} \mathcal{C}_{x_{0}} M$, for some choice of a Riemannian metric on $T M$ extending that of $E$. Then there is a section $\alpha$ of the vector bundle $L^{2} T \mathcal{C}_{x_{0}} M \rightarrow \mathcal{C}_{x_{0}} M$ such that if $u \in L^{2} T_{\sigma} \mathcal{C}_{x_{0}} M$ then

$$
\phi(u)=\int_{0}^{T}\left\langle\alpha(\sigma)_{t}, u_{t}\right\rangle_{\sigma(t)} d t .
$$

Assume $\alpha$ is in $L^{2}$, i.e. $\int_{\mathcal{C}_{x_{0}} M}\|\alpha(\sigma)\|_{L^{2}}^{2} d \mu_{x_{0}}(\sigma)<\infty$. We first show that for such $\phi$ the right hand side of (3.8) makes sense and agrees with the pull back $\phi(v$.). It is easy to verify that

$$
\phi_{t}^{\#}=\mathbf{W}_{t}\left(\Pi\left(W^{-1}\right)^{*} \int^{T} W_{s}^{*} \alpha_{s} d s\right),
$$

where $W_{r}^{*}: T_{x_{r}} M \rightarrow T_{x_{0}} M$ is the adjoint of $W_{r}$. Let $e^{1}, \ldots, e^{n-p}$ be an orthonormal base for $\operatorname{ker}\left[X\left(x_{0}\right)\right]$. Set $e_{s}^{j}=\tilde{/}_{s} e^{j}$ and $\beta_{s}^{j}=\left\langle\beta_{s}, e^{j}\right\rangle_{\mathbf{R}^{m}}$. Then

$$
\begin{aligned}
\Phi(h, \phi) & =\sum_{j} \phi\left(W \cdot \int_{0}^{T} \chi_{[0, \cdot]}(s) W_{s}^{-1} \nabla_{v_{s}} X\left(e_{s}^{j}\right) d \beta_{s}^{j}\right) \\
& =\sum_{j} \int_{0}^{T} \phi\left(\chi_{[0, \cdot]}(s) W^{s} \nabla_{v_{s}} X\left(e_{s}^{j}\right)\right) d \beta_{s}^{j}
\end{aligned}
$$


where $W_{t}^{s}=W_{t}^{-1} W_{s}$, since $\phi_{x}$. is $\mathcal{F}^{x_{0}}=\mathcal{G}_{0}$ measurable. Using (3.15) this gives

$$
\begin{aligned}
\Phi(h, \phi) & =\sum_{j} \int_{0}^{T}\left\{\int_{s}^{T}\left\langle\alpha_{t}, W_{t}^{s} \nabla_{v_{s}} X\left(e_{s}^{j}\right) d t\right\rangle\right\} d \beta_{s}^{j} \\
& =\int_{0}^{T}\left\langle\int_{s}^{T}\left(W_{t}^{s}\right)^{*} \alpha_{t} d t, \nabla_{v_{s}} X\left(\tilde{I}_{s} d \beta_{s}\right)\right\rangle_{x_{s}} \\
& =\int_{0}^{T}\left\langle\frac{\mathbb{D}}{d s} \phi_{s}^{\#}, \nabla_{v_{s}} X\left(\tilde{/}{ }_{s} d \beta_{s}\right)\right\rangle_{x_{s}} .
\end{aligned}
$$

This shows that $\phi(v$. $)=\mathcal{I}^{*}(\phi)$ agrees with the right hand side of (3.8), as expected.

(3) In general for $\psi$ a measurable $\mathcal{H}$-form define

$$
\tilde{\Phi}(\psi)_{t}:=\int_{0}^{t}\left\langle\frac{\mathbb{D}}{d s} \psi_{s}^{\#}, \nabla_{T \mathcal{I}_{s}(-)} X\left(\tilde{/}_{s} d \beta_{s}\right)\right\rangle_{x_{s}}, \quad 0 \leq t \leq T .
$$

Note that $\left(\tilde{\Phi}(\psi)_{t}, 0 \leq t \leq T\right)$ is a local $\mathcal{G}_{*}$-martingale with values in $H^{*}$ since

$$
\int_{0}^{T} \sup _{\|h\|_{H} \leq 1}\left|\left(\nabla_{T \mathcal{I}_{s}(h)} X\right)^{*}\left(\frac{\mathbb{D}}{d s} \psi_{s}^{\#}\right)\right|_{x_{s}}^{2} d s<\infty, \quad \text { a.s. }
$$

using the fact that

$$
\sup _{0 \leq s \leq T} \sup _{\|h\|_{H} \leq 1}\left|\left(\nabla_{T \mathcal{I}_{s}(h)} X\right)\right|_{\mathbb{L}\left(\mathbf{R}^{m} ; E_{x_{s}}\right)}^{2}<\infty \quad \text { a.s. . }
$$

The usual stopping time argument, see Lemma 3.1, shows that $\tilde{\Phi}(\psi)(-)$ is a continuous map from $L^{0} \Gamma \mathcal{H}^{*}$ to $L^{0}\left(\mathcal{C}_{0} \mathbf{R}^{m} ; H^{*}\right)$.

To see that the pull back map of a geometric differential 1-form $\phi$ evaluated at $h$ agrees with the right hand side of (3.8), we only need to show that $\tilde{\Phi}(\phi)(h)=\Phi(h, \phi)$. For this define a sequence of differential forms $\phi_{n}$, which extends over $L^{2} T C_{x_{0}} M$, by

$$
\phi_{n}(\sigma)(u)=\phi\left(/ / .(\sigma) \int_{0}^{T} \lambda_{n}(s-\cdot) / /_{s}^{-1}(\sigma) u_{s} d s\right)
$$

for suitable $\lambda_{n}: \mathbf{R}^{n} \rightarrow \mathbf{R}^{n}, n=1,2, \ldots$ so that $\phi_{n}$ converges to $\phi$ on $\left(T_{\sigma} C_{x_{0}} M\right)^{*}$ for almost all $\sigma$, and observe that $\tilde{\Phi}\left(\phi_{n}\right)(h) \rightarrow \tilde{\Phi}(\phi)(h)$ in particular from the convergence of $\phi_{n}$ in $\mathcal{H}^{*}$ and Lemma 3.1.

Furthermore by the Burkholder-Davis-Gundy inequalities, [8], for $0<p<\infty$

$$
\begin{aligned}
\mathbf{E}|\tilde{\Phi}(\psi)|^{p} & \leq\left.\left. C_{p} \mathbf{E}\left|\int_{0}^{T}\right|\left(\nabla_{T \mathcal{I}_{s}(-)} X\right)^{*}\left(\frac{\mathbb{D}}{d s} \psi_{s}^{\#}\right)\right|_{\mathbb{L}_{2}\left(H ; \mathbf{R}^{m}\right)} ^{2} d s\right|^{p / 2} \\
& \leq C_{p} \mathbf{E}\left\{\sup _{0 \leq s \leq T}\left|\left(\nabla_{T \mathcal{I}_{s}(-)} X\right)^{*}\right|_{\mathbb{L}_{2}\left(H ; \mathbb{L}_{\left.\left(E_{x_{s}} ; \mathbf{R}^{m}\right)\right)}^{p}\|\psi\|_{\mathcal{H}^{*}}^{p}\right\} .}\right.
\end{aligned}
$$

For $1 \leq p<\infty$ this gives by Hölder's inequality, for $\epsilon>0$,

$$
|\tilde{\Phi}(\psi)|_{L^{p}} \leq \text { const. }\left.\left.\|\psi\|_{L^{p+\epsilon}} \cdot\left|\sup _{0 \leq s \leq T}\right| T \mathcal{I}_{s}(-)\right|_{\mathbb{L}\left(H ; T_{x_{s}} M\right)}\right|_{L} \frac{p(p+\epsilon)}{\epsilon} .
$$


Since $\sup _{0 \leq s \leq T}\left|T \mathcal{I}_{s}(-)\right|$ lies in $L^{q}$ for all $1 \leq q<\infty$ we see that $\tilde{\Phi}$ gives a continuous linear map

$$
\tilde{\phi}: L^{p+\epsilon} \Gamma \mathcal{H}^{*} \longrightarrow L^{p}\left(\mathcal{C}_{0} \mathbf{R}^{m} ; H^{*}\right)
$$

for all $1 \leq p<\infty$ and $\epsilon>0$.

Combining the two terms in (3.13) we obtain a continuous linear map

$$
\psi \mapsto \int_{0}^{T}\left\langle\frac{\mathbb{D}}{d t} \psi_{t}^{\#}, \nabla_{T \mathcal{I}_{t}(-)} X\left(\tilde{/} / t d \beta_{t}\right)+X\left(x_{t}\right)\left(\frac{d}{d t}-\right)\right\rangle_{x_{t}} d t
$$

from $L^{p+\epsilon} \Gamma \mathcal{H}^{*}$ to $L^{p}\left(\mathcal{C}_{0} \mathbf{R}^{m} ; H^{*}\right)$ in the relevant range of $1 \leq p<\infty, \epsilon>0$, which agrees with

$$
\psi \mapsto \mathcal{I}^{*}(\psi)
$$

when $\psi$ is an $L^{\infty}$ section of $\left(L^{2} T \mathcal{C}_{x_{0}} M\right)^{*}$.

Supposing furthermore that condition $\left(M_{0}\right)$ holds, observe that (3.17) gives

$$
\|\tilde{\Phi}(\psi)\|_{L^{p}} \leq\left(C_{p}\right)^{1 / p}\|\psi\|_{L^{p}} \cdot \mid \mathbf{E}\left\{\sup _{0 \leq s \leq T}\left|\nabla_{T \mathcal{I}_{s}(-)} X\right|^{p} \mid \mathcal{F}^{x_{0}}\right\} \|_{L^{\infty}}
$$

with

$$
\begin{aligned}
& \mathbf{E}\left\{\sup _{0 \leq s \leq T}\left|\nabla_{T \mathcal{I}_{s}(-)} X\right|^{p} \mid \mathcal{F}^{x_{0}}\right\} \\
& \leq \sup _{0 \leq s \leq T}\left|\nabla_{W_{s}(-)} X\right|_{\mathbb{L}\left(T_{x_{0} M}^{p} ; \mathbb{L}\left(\mathbf{R}^{m} ; E_{x_{s}}\right)\right)}^{p} \mathbf{E}\left\{\sup _{0 \leq s \leq T}\left|W_{s}^{-1} T \mathcal{I}_{s}(-)\right|_{\mathbb{L}_{2}\left(H ; T_{\left.x_{0} M\right)}\right)}^{p} \mid \mathcal{F}^{x_{0}}\right\}
\end{aligned}
$$

which is essentially bounded by condition $\left(M_{0}\right)$ and Proposition 3.3. Thus in this case we can take $\epsilon=0$ and (a) is proved.

To see that $\mathcal{I}^{*}: L^{0} \rightarrow L^{0}$ is injective note that by (3.8) and the independence of $\beta$ and $\mathcal{F}^{x_{0}}$,

$$
\mathbf{E}\left\{\mathcal{I}^{*}(\phi(-)) \mid \mathcal{F}^{x_{0}}\right\}=\int_{0}^{T}\left\langle\frac{\mathbb{D}}{d s} \phi_{s}^{\#}, X\left(x_{s}\right)\left(\frac{d}{d s}-\right)\right\rangle_{x_{s}} d s=\phi\left(\overline{T \mathcal{I}}_{x .}-\right)
$$

(with a suitable interpretation of the conditional expectation if $\mathcal{I}^{*}(\phi)$ is not in $L^{1}$, e.g. see Elworthy-LeJan-Li [15] p66). Using the inverse of $\overline{T \mathcal{I}}$, see Property 3.1 we obtain

$$
\phi_{x .}(-)=\mathbf{E}\left\{\mathcal{I}^{*}(\phi)\left(\int_{0} Y_{x_{s}}\left(\frac{\mathbb{D}}{d s}-\right) d s\right) \mid \mathcal{F}^{x_{0}}\right\} .
$$

This proves injectivity, giving a left inverse for $\mathcal{I}^{*}$.

Moreover since $v \mapsto \int_{0}^{\cdot} Y_{x_{s}}\left(\frac{\mathbb{D}}{d s} v_{s}\right) d s$ is an isometry of $\mathcal{H}_{x}$. $\rightarrow H$ almost surely we see

$$
\begin{aligned}
\|\phi\|_{L^{p}} & =\left\|\mathbf{E}\left\{\mathcal{I}^{*}(\phi) \mid \mathcal{F}^{x_{0}}\right\}\right\|_{L^{p}} \\
& \leq\left\|\mathcal{I}^{*}(\phi)\right\|_{L^{p}}
\end{aligned}
$$

completing the proof.

Remark 3.5 Although the term $\frac{\mathbb{D}}{d s} \phi_{s}^{\#}$ appearing in (3.8) may depend on the whole path $\left\{x_{s}: 0 \leq s \leq T\right\}$, the stochastic integral there can and was considered as an Itô integral by regarding $\beta$. as a martingale with respect to $\mathcal{F}^{x_{0}} \cup \mathcal{F}_{t}$. We can also treat it 
as a Skorohod integral, c.f. X-D Li [34] for pull backs by the stochastic development map. In fact if $\phi \in L^{p+\epsilon} \Gamma \mathcal{H}$ the stochastic integral can be written as

$$
\int_{0}^{T}\left\langle\nabla_{T \mathcal{I}_{s}(-)} Y\left(\frac{\mathbb{D}}{d s} \phi_{s}^{\#} \circ \mathcal{I}\right), d B_{s}\right\rangle
$$

and interpreted as a Skorohod integral on $\mathcal{C}_{0} \mathbf{R}^{m}$, i.e. as

$$
\left(d^{q}\right)^{*}\left(\alpha \mapsto \int_{0}^{T} \chi_{[0, \cdot]}(s)\left\langle\nabla_{T \mathcal{I}_{s}(-)} Y\left(\frac{\mathbb{D}}{d s} \phi_{s}^{\#} \circ \mathcal{I}\right), \frac{d}{d s} \alpha_{s}\right\rangle_{\mathbf{R}^{m}} d s\right)
$$

for $\frac{1}{p}+\frac{1}{q}=1$ when $1<p<\infty$. Here

$$
d^{q} \equiv d_{H^{*}}^{q}: \operatorname{Dom}\left(d^{q}\right) \subset L^{q}\left(\mathcal{C}_{0} \mathbf{R}^{m} ; H^{*}\right) \longrightarrow L^{q}\left(\mathcal{C}_{0} \mathbf{R}^{m} ; \mathbb{L}_{2}\left(H ; H^{*}\right)\right)
$$

Proof Set $G=\mathbb{L}_{2}\left(H ; L_{0}^{2,1}\left([0, T] ; T_{x_{0}} M\right)\right)$. Define $\theta: \Omega \rightarrow \mathbb{L}_{2}(H ; G)$ by

$$
\theta(\alpha)(h)_{\tau}=\int_{0}^{T} \chi_{[0, \tau]}(s) W_{s}^{-1} \nabla_{T_{\mathcal{I}}(h)} X\left(\dot{\alpha}_{s}\right) d s, \quad \alpha, h \in H, 0 \leq \tau \leq T .
$$

Then

$$
\left(d_{G}^{q}\right)^{*}(\theta)(-)_{\tau}=\int_{0}^{T} \chi_{[0, \tau]}(s) W_{s}^{-1} \nabla_{T \mathcal{I}_{s}(-)} X\left(d B_{s}\right) \in \mathbb{L}\left(H ; T_{x_{0}} M\right) .
$$

Suppose first that $\phi$ is smooth and cylindrical. Then

$$
\Phi(-, \phi)=\phi_{x}\left(W . \int_{0}^{T} \chi_{[0, \cdot]}(s) W_{s}^{-1} \nabla_{T \mathcal{I}_{s}(-)} X\left(d B_{s}\right)\right)=\phi_{x}\left(W .\left(d_{G}^{q}\right)^{*}(\theta)(-) .\right) .
$$

Now, if $\left\{E^{k}\right\}_{k=1}^{\infty}$ is an orthonormal base for $H, g: \mathcal{C}_{0} \mathbf{R}^{m} \rightarrow \mathbf{R}$ is $C^{\infty}$ smooth cylindrical, and $h \in H$,

$$
\begin{aligned}
& \mathbf{E}\left[g \phi_{x .}\left(W .\left(d_{G}^{q}\right)^{*} \theta(-) .\right)(h)\right]=\mathbf{E}\left[g \phi_{x .}\left(W .\left(d_{L_{0}^{2,1}\left([0, T] ; T_{x_{0}} M\right)}^{q}\right)^{*}(\theta(-)(h))\right)\right] \\
& =\mathbf{E}\left[\sum_{j} d^{q}\left(g \phi_{x .}(W .-)\right)\left(E^{j}\right)\left(\theta\left(E^{j}\right)(h) .\right)\right] \\
& =\mathbf{E}\left[\sum_{j} d^{q} g\left(E^{j}\right) \phi_{x .}\left(W . \theta\left(E^{j}\right)(h) .\right)\right]+\mathbf{E}\left[\sum_{j} g d^{q}\left(\phi_{x .}(W .-)\right)\left(E^{j}\right)\left(\theta\left(E^{j}\right)(h) .\right)\right] \\
& =\mathbf{E}\left\langle d^{q} g, \phi_{x .}(W . \theta(-)(h) .\rangle_{H^{*}}+\mathbf{E}\left[\sum_{j} g d^{q}\left(\phi_{x .}(W .-)\right)\left(E^{j}\right)\left(\theta\left(E^{j}\right)(h) .\right)\right] .\right.
\end{aligned}
$$

Furthermore

$$
\begin{aligned}
& \sum_{j} d\left(\phi_{x .}(W .-)\right)\left(E^{j}\right)\left(\theta\left(E^{j}\right)(h) .\right) \\
& =\sum_{j} d\left(\phi_{x .}(W .-)\right)\left(\int_{0}^{\cdot} K^{\perp}\left(x_{s}\right) \dot{E}_{s}^{j} d s\right)\left(\theta\left(\int_{0}^{.} K\left(x_{s}\right) E_{s}^{j} d s\right)(h)\right),
\end{aligned}
$$


since $T \mathcal{I}\left(E^{j}\right)=T \mathcal{I}\left(\int_{0}^{T} K^{\perp}\left(x_{s}\right) \dot{E}_{s}^{j} d s\right)$ and $\nabla_{T \mathcal{I}_{s}(\cdot)} X\left(\dot{E}_{s}^{j}\right)=\nabla_{T \mathcal{I}_{s}(\cdot)} X\left(K\left(x_{s}\right)\left(\dot{E}_{s}^{j}\right)\right)$ by the defining property of $\nabla$, Proposition 2.1. Note that the expression is independent of the choice of basis and so vanishes giving

$$
\Phi(h, \phi)=\phi_{x}\left(W .\left(d_{G}^{q}\right)^{*}(\theta(-) .(h))\right)=\left(d_{H^{*}}^{q}\right)^{*}\left(\phi_{x .}(W . \theta(-)(h) .)\right) .
$$

Note that for $h \in H$,

$$
\phi_{x .}(W . \theta(-)(h))_{t}^{\#}=\int_{0}^{t} \nabla_{T \mathcal{I}_{s}(h)} Y\left(\frac{\mathbb{D}}{d s} \phi_{s}^{\#}\right) d s \in H,
$$

to obtain the desired result.

For general $\phi \in L^{p+\epsilon} \Gamma \mathcal{H}$ we can take $C^{\infty}$ cylindrical one-forms $\phi^{j}, j=1$ to $\infty$, converging to $\phi$ in $L^{p+\epsilon} \Gamma \mathcal{H}$. By the Theorem, $\mathcal{I}^{*}\left(\phi^{j}\right) \rightarrow \mathcal{I}^{*}(\phi)$ in $L^{p}$ and we see therefore that $\Phi\left(-, \phi^{j}\right)$ is convergent in $L^{p}$. Thus $\nabla_{T \mathcal{I}_{s}(-)} Y_{-}\left(\frac{\mathbb{D}}{d s} \phi_{s}^{\#}\right)$ is in the domain of $\left(d^{q}\right)^{*}$ and the result holds.

Remark 3.6 From (3.8) we see that $\mathcal{I}^{*}(\lambda \phi)=\lambda \mathcal{I}^{*}(\phi)$ for all $\phi \in L^{0} \Gamma \mathcal{H}^{*}$, if $\lambda \in$ $L^{0}\left(\mathcal{C}_{x_{0}} M ; \mathbf{R}\right)$. This is because $\lambda \circ \mathcal{I} \in \mathcal{G}_{0}$ for all such $\lambda$. From the Skorohod integral representation,

$$
\mathcal{I}^{*}(\phi)=\int_{0}^{T}\left\langle\nabla_{T \mathcal{I}_{s}(-)} Y\left(\frac{\mathbb{D}}{d s} \phi_{s}^{\#} \circ \mathcal{I}\right), d B_{s}\right\rangle+\int_{0}^{T}\left\langle\frac{\mathbb{D}}{d s} \phi_{s}^{\#}, X\left(x_{s}\right) \dot{h}_{s}\right\rangle d s,
$$

this is less obvious. However for sufficiently regular $\lambda$,

$$
\begin{aligned}
& \int_{0}^{T}\left\langle\nabla_{T \mathcal{I}_{s}(-)} Y\left(\frac{\mathbb{D}}{d s}\left(\lambda \phi_{s}^{\#}\right) \circ \mathcal{I}\right), d B_{s}\right\rangle \\
= & \lambda \int_{0}^{T}\left\langle\nabla_{T \mathcal{I}_{s}(-)} Y\left(\frac{\mathbb{D}}{d s} \phi_{s}^{\#} \circ \mathcal{I}\right), d B_{s}\right\rangle \\
& -\int_{0}^{T}\left\langle\nabla_{T \mathcal{I}_{s}(-)} Y\left(\frac{\mathbb{D}}{d s} \phi_{s}^{\#} \circ \mathcal{I}\right), \frac{d}{d s} \nabla(\lambda \circ \mathcal{I})\right\rangle_{x_{s}},
\end{aligned}
$$

and as for the proof of Remark 3.5 the second term vanishes.

For suitable $h: \mathcal{C}_{0} \mathbf{R}^{m} \rightarrow H$ define a measurable vector field $\overline{T \mathcal{I}(h)}$ on $\mathcal{C}_{x_{0}} M$ by

$$
\overline{T \mathcal{I}(h)}(\sigma)=\mathbf{E}\{T \mathcal{I}(h(\cdot)) \mid \mathcal{I}(\cdot)=\sigma\}
$$

for $\mu_{x_{0}}$-almost all $\sigma$ in $\mathcal{C}_{x_{0}} M$. Note that if $h$ is $\mathcal{F}^{x_{0}}$ measurable with $h=\bar{h} \circ \mathcal{I}$ then $\overline{T \mathcal{I}(h)}(\sigma)=\overline{T \mathcal{I}}_{\sigma}(\bar{h}(\sigma))$ for $\overline{T \mathcal{I}}$ as in Property 3.1. For completeness we give the following extension of Theorem 2.2 of Elworthy-Li [19]:

Corollary 3.7 For $\delta>0$ and $1<q<\infty$ the map $h \mapsto \overline{T \mathcal{I}(h)}$ gives a continuous linear map

$$
\overline{T \mathcal{I}(-)}: L^{q}\left(\mathcal{C}_{0} \mathbf{R}^{m} ; H\right) \rightarrow L^{q-\delta} \Gamma \mathcal{H} .
$$

(This map is the co-joint of $\mathcal{I}^{*}$ in the sense that

$$
\int_{\mathcal{C}_{0} \mathbf{R}^{m}} \mathcal{I}^{*}(\phi)(h) d P=\int_{\mathcal{C}_{0} \mathbf{R}^{m}} \phi(\overline{T \mathcal{I}(h)}) d P
$$

for $\phi \in L^{p+\epsilon} \Gamma \mathcal{H}^{*}$ and $h \in L^{q}\left(\mathcal{C}_{0} \mathbf{R}^{m} ; H\right)$, taking $\epsilon>0$ and $\frac{1}{p}+\frac{1}{q}=1$.)

If Condition $\left(M_{0}\right)$ holds we can allow $\delta=0$ and also $q=\infty$, and $\overline{T \mathcal{I}(-)}$ : $L^{q}\left(\mathcal{C}_{0} \mathbf{R}^{m} ; H\right) \rightarrow L^{q} \Gamma \mathcal{H}$ is surjective, $1<q \leq \infty$. 
Proof First note that when $\phi$ is a true form

$$
\int_{\mathcal{C}_{0} \mathbf{R}^{m}} \mathcal{I}^{*}(\phi)(h) d P=\mathbf{E}\left\{\phi\left(\mathbf{E}\left\{T \mathcal{I}(h) \mid \mathcal{F}^{x_{0}}\right\}\right)\right\}=\mathbf{E} \phi(\overline{T \mathcal{I}(h)})
$$

so (3.20) holds and it holds for $\phi \in L^{p+\epsilon} \Gamma \mathcal{H}^{*}, h \in L^{q}\left(\mathcal{C}_{0} \mathbf{R}^{m} ; H\right)$ for $\frac{1}{p}+\frac{1}{q}=1$ by continuity, from Theorem 3.4. Consequently for such $\phi$ and $h$,

$$
\left|\int_{\mathcal{C}_{0} \mathbf{R}^{m}} \phi(\overline{T \mathcal{I}(h)}) d P\right| \leq\left\|\left.\mathcal{I}^{*}(\phi)\right|_{L^{p}} \cdot\right\| h \|_{L^{q}} \leq \text { const. } \cdot \mid \phi\left\|_{L^{p+\epsilon}}\right\| h \|_{L^{q}}
$$

Since this holds for all $\phi$ in $L^{p+\epsilon}$ we see that $\overline{T \mathcal{I}(h)} \in L^{\frac{p+\epsilon}{p+\epsilon-1}}$ and is continuous linear in $h$ into $L^{\frac{p+\epsilon}{p+\epsilon-1}}$. Thus if $h \in L^{q}$ then $\overline{T \mathcal{I}(h)} \in L^{\frac{p+\epsilon}{p+\epsilon-1}}$ for all $\epsilon>0$ so that $\overline{T \mathcal{I}(-)}$ is continuous linear from $L^{q}$ to $L^{q-\delta}$ for any $\delta>0$, with $\delta=0$ allowed if condition $\left(M_{0}\right)$ holds.

Surjectivity comes from the fact that $\overline{T \mathcal{I}(-)}$ has a right inverse

$$
v \mapsto \int_{0}^{\cdot} Y_{x_{s}}\left(\frac{\mathbb{D}}{d s} v_{s} \circ \mathcal{I}\right) d s
$$

mapping $L^{q} \Gamma \mathcal{H}$ to $L^{q}\left(\mathcal{C}_{0} \mathbf{R}^{m} ; H\right)$ as in Property 3.1.

Analogously to (3.16) we have also:

Remark 3.8 Let $N:[0, T] \times \mathcal{C}_{x_{0}} M \rightarrow \mathbf{R}^{p}$ be a continuous semi-martingale on the filtered probability space $\left\{\mathcal{C}_{x_{0}} M, \mu_{x_{0}}, \mathcal{F}_{*}^{x_{0}}\right\}$ where $\mathcal{F}_{t}^{x_{0}}=\sigma\left\{x_{s}: 0 \leq s \leq t\right\}$. Suppose $\alpha$. is a locally bounded section of $\mathbb{L}\left(\mathbf{R}^{p} ; L^{2} T \mathcal{C}_{x_{0}} M\right)$ which is $\mathcal{F}_{*}^{x_{0}}$ adapted. Then the mapping

$$
\begin{aligned}
L^{2} \mathcal{H} & \rightarrow L^{0}\left(\mathcal{C}_{x_{0}} M ; \mathbf{R}\right) \\
U & \mapsto \int_{0}^{T}\left\langle U(\sigma), \alpha(\sigma)_{s} d N_{s}(\sigma)\right\rangle_{\sigma(s)}
\end{aligned}
$$

can be considered as the map $U \mapsto \varphi(U(\cdot))$ for $\varphi$ the $H$-one-form determined by the $\mathcal{H}$-vector field $\varphi^{\#}$ with

$$
\varphi_{t}^{\#}=\mathbf{W}_{t}\left(\Pi\left(W^{-1}\right)^{*} \int_{.}^{T} W_{s}^{*} \alpha_{s} d N_{s}\right), \quad 0 \leq t \leq T .
$$

If the martingale part of $N$. is zero then no adapteness is required.

Remark 3.9 The proof of Theorem 2.2 in Elworthy-Li [19] as it stands has a trivial mistake in the last line and only gives $\overline{T \mathcal{I}(h)} \in L^{1}$ when $h \in L^{2}$. However it is easily modified by not taking expectations in the proof of Lemma 3.3 of [19]: this shows that $\overline{T \mathcal{I}(h)} \in L^{\infty}$ if $h \in L^{2}\left(\mathcal{C}_{0} \mathbf{R}^{m}, \sigma\left\{\beta_{s}: 0 \leq s \leq T\right\} ; H\right)$, not just $L^{2}$, using the observation that $\sup _{0<r<T} \mathbf{E}\left\{\left|T \mathcal{I}_{r}\right|^{2} \mid \mathcal{F}^{x_{0}}\right\}<\infty$ from Aida-Elworthy [2], i.e. a special case of Proposition 3.3 above. 


\section{Sobolev calculus on $\mathcal{C}_{x_{0}} M$ and its intertwining by Itô maps}

If $M$ is given a Riemannian metric then $\mathcal{C}_{x_{0}} M$ gets a Finsler structure defined by

$$
\|v\|_{\sigma}:=\sup _{0 \leq t \leq T}\left|v_{t}\right|_{\sigma(t)}, \quad v \in T_{\sigma} \mathcal{C}_{x_{0}} M
$$

By the compactness of $M$ different metrics produce uniformly equivalent Finsler norms. From Elworthy-LeJan-Li [15] and Elworthy-Ma [26] and the compactness of $M$, for almost all $\sigma$ the inclusion $\mathcal{H}_{\sigma} \hookrightarrow T_{\sigma} \mathcal{C}_{x_{0}} M$ is continuous and its norm is in $L^{p}$ as a function of $\sigma$ for all $p \in[1, \infty)$ and is essentially bounded for any of these Finsler norms if condition (M) holds.

A function $f: \mathcal{C}_{x_{0}} M \rightarrow \mathbf{R}$ will be said to be $B C^{1}$ if it is Fréchet differentiable and is bounded together with its differential $d f$ considered as a section of $T^{*} \mathcal{C}_{x_{0}} M$, again using any of these Finsler structures. Note that then $d f$ restricts to $\mathcal{H}$ to give an element of $L^{p} \Gamma \mathcal{H}^{*}$ for all $1<p<\infty$ and lies in $L^{\infty} \Gamma \mathcal{H}^{*}$ if condition $M$ holds, by the definition of $\mathcal{H}$ and Proposition 2.2.

Denote by $\mathrm{Cyl}$ the space of smooth cylindrical functions on $\mathcal{C}_{x_{0}} M$. Let $\operatorname{Dom}\left(d_{\mathcal{H}}\right)$ be a linear subspace of $L^{\infty}\left(\mathcal{C}_{x_{0}} M ; \mathbf{R}\right)$ with

$$
\operatorname{Cyl} \subset \operatorname{Dom}\left(d_{\mathcal{H}}\right) \subset B C^{1} .
$$

Define

$$
d=d_{\mathcal{H}}: \operatorname{Dom}\left(d_{\mathcal{H}}\right) \rightarrow \cap_{1 \leq p<\infty} L^{p} \Gamma \mathcal{H}^{*}
$$

by restriction:

$$
\left(d_{\mathcal{H}} f\right)_{\sigma}=\left.d f_{\sigma}\right|_{\mathcal{H}_{\sigma}} .
$$

Let $\operatorname{Dom}\left(d_{\mathcal{H}}\right)$ be the space of equivalence classes of $\operatorname{Dom}\left(d_{\mathcal{H}}\right)$ under equality up to sets of $\mu_{x_{0}}$-measure zero. However after this section we will not distinguish between $\operatorname{Dom}(d)$ and $\mathcal{D} \operatorname{om}(d)$. By a standard result the set of smooth cylindrical functions is dense in $L^{p}\left(\mathcal{C}_{x_{0}} M ; \mathbf{R}\right), 1 \leq p<\infty$, and therefore so is $\mathcal{D o m}\left(d_{\mathcal{H}}\right)$.

We next give the proof of closability of $d_{\mathcal{H}}$ restricted to $\mathcal{D}$ om $\left(d_{\mathcal{H}}\right)$ in our context. For classical Wiener space this is one of the basic results of Malliavin calculus and gives for each $1 \leq p<\infty$ a closed linear operator

$$
d \equiv d^{p}: \mathcal{D} \operatorname{om}\left(d^{p}\right) \subset L^{p}\left(\mathcal{C}_{0} \mathbf{R}^{m} ; \mathbf{R}\right) \rightarrow L^{p}\left(\mathcal{C}_{0} \mathbf{R}^{m} ; H^{*}\right)
$$

This is proved by the standard method of integration by parts in Nualart [38]. (Strictly speaking his basic domain does not consist of $B C^{1}$ functions but it is easy to see, and well known, that this gives the same closures $d^{p}$.) On the path space $d_{\mathcal{H}}$ is closable, from Elworthy-LeJan-Li [15], in $L^{2}\left(\mathcal{C}_{x_{0}} M ; \mathbf{R}\right)$. For $p>1$ the following theorem follows in the same way from the integration by parts results in Elworthy-LeJan-Li [16], [15]. Related results in this context and a detailed discussion of the Dirichlet forms which arise can be found in Elworthy-Ma [26] and [22]. See also Driver [9] and Hsu [29].

Theorem 4.1 For $1 \leq p<\infty$ the operator $d_{\mathcal{H}}$ can be considered as a linear operator

$$
d_{\mathcal{H}}: \operatorname{Dom}\left(d_{\mathcal{H}}\right) \subset L^{p}\left(\mathcal{C}_{x_{0}} M ; \mathbf{R}\right) \rightarrow L^{p} \Gamma \mathcal{H}^{*}
$$

It is closable for each $1<p<\infty$ and for $p=1$ if condition $\left(M_{0}\right)$ holds. 
Proof It is not immediately obvious that $d_{\mathcal{H}}$ is defined on $\mathcal{D} \operatorname{om}\left(d_{\mathcal{H}}\right)$ : we need to know that if $f \in \operatorname{Dom}\left(d_{\mathcal{H}}\right)$ is $\mu_{x_{0}}$-almost surely 0 then $d_{\mathcal{H}} f=0$ almost surely (c.f. Proposition 3.5 in Elworthy-Ma [26]). This comes together with closability from the following proof that if $f_{j} \in \mathcal{D o m}\left(d_{\mathcal{H}}\right)$ has $f_{j} \rightarrow 0$ in $L^{p}$ and $d_{\mathcal{H}} f_{j} \rightarrow \theta$ in $L^{p} \Gamma \mathcal{H}^{*}$ then $\theta=0$. For this note that $f_{j} \circ \mathcal{I} \rightarrow 0$ in $L^{p}\left(\mathcal{C}_{0} \mathbf{R}^{m} ; \mathbf{R}\right)$ and $f_{j} \circ \mathcal{I}$ belongs to the domain of $d$, as is well known, for example by Wong-Zakai approximations. Moreover

$$
d^{p}\left(f_{j} \circ \mathcal{I}\right)=d_{\mathcal{H}} f_{j} \circ T \mathcal{I}=\mathcal{I}^{*}\left(d_{\mathcal{H}} f_{j}\right)
$$

By Theorem $3.4, \mathcal{I}^{*}\left(d_{\mathcal{H}} f_{j}\right)$ converges to $\mathcal{I}^{*}(\theta)$ in $L^{p^{\prime}}$ for any $p^{\prime} \in[1, p)$ and for $p^{\prime}=p=1$ if condition $\left(M_{0}\right)$ holds. Since $d^{p^{\prime}}$ for $\mathcal{C}_{0} \mathbf{R}^{m}$ is closed this shows that $\mathcal{I}^{*}(\theta)=0$. But Theorem 3.4 shows $\mathcal{I}^{*}$ is injective and so $\theta=0$ as required.

Let $d^{p}: \operatorname{Dom}\left(d^{p}\right) \subset L^{p}\left(\mathcal{C}_{x_{0}} M ; \mathbf{R}\right) \rightarrow L^{p} \Gamma \mathcal{H}^{*}, 1 \leq p<\infty$, be the closures of $d_{\mathcal{H}}$ given by Theorem 4.1 .

We now comes to our main result on the chain rule, or intertwining. For $1 \leq p^{\prime} \leq$ $p<\infty$ let

$$
\mathcal{I}^{*}: L^{p}\left(\mathcal{C}_{x_{0}} M ; \mathbf{R}\right) \rightarrow L^{p}\left(\mathcal{C}_{0} \mathbf{R}^{m} ; \mathbf{R}\right) \hookrightarrow L^{p^{\prime}}\left(\mathcal{C}_{0} \mathbf{R}^{m} ; \mathbf{R}\right)
$$

denote the map $\mathcal{I}^{*} f=f \circ \mathcal{I}$ as well as the map from $L^{p} \Gamma \mathcal{H}^{*}$ to $L^{p^{\prime}}\left(\mathcal{C}_{0} \mathbf{R}^{m} ; H^{*}\right)$, as defined by Theorem 3.4. We will see below that the Itô map for our s.d.e. can be used in some sense as a substitute for a chart for the 'differential structure' given by the calculus. See also Aida-Elworthy [2], Aida [1], Elworthy-Li- [19] for the gradient case, Elworthy-LeJan-Li [15], Elworthy-Li [20] more generally, and for related work see Fang-Franchi [27], [34] and Cruzeiro-Malliavin [6].

Theorem 4.2 Suppose $1 \leq p^{\prime} \leq p<\infty$, with $1<p^{\prime}<p$ unless condition $\left(M_{0}\right)$ holds. The operators

$$
\mathcal{I}^{*} d^{p}: \mathcal{D} \operatorname{om}\left(d^{p}\right) \subset L^{p}\left(\mathcal{C}_{x_{0}} M ; \mathbf{R}\right) \longrightarrow L^{p^{\prime}}\left(\mathcal{C}_{0} \mathbf{R}^{m} ; H\right)
$$

and

$$
d^{p^{\prime}} \mathcal{I}^{*}:\left\{f \in L^{p} \mid \mathcal{I}^{*} f \in \mathcal{D} \operatorname{om}\left(d^{p^{\prime}}\right)\right\} \subset L^{p}\left(\mathcal{C}_{x_{0}} M ; \mathbf{R}\right) \longrightarrow L^{p^{\prime}}\left(\mathcal{C}_{0} \mathbf{R}^{m} ; H\right)
$$

are densely defined. Moreover

(i) $\mathcal{I}^{*} d^{p}$ is closable in general and closed if condition $\left(M_{0}\right)$ holds and $p=p^{\prime}$;

(ii) $d^{p^{\prime}} \mathcal{I}^{*}$ is closed.

(iii)

$$
\mathcal{I}^{*} d^{p} \subset d^{p^{\prime}} \mathcal{I}^{*}
$$

Proof The denseness of the domain of $d^{p^{\prime}} \mathcal{I}^{*}$ and the fact that it is closed are automatic by continuity of $\mathcal{I}^{*}$, giving (ii). It is clear that $\mathcal{I}^{*} d^{p}$ is densely defined.

For (i) first suppose that condition $\left(M_{0}\right)$ holds and $p=p^{\prime}$. Then $\mathcal{I}^{*}$ on $L^{p} \mathcal{H}$-forms is continuous and has closed range, by Theorem 3.4. It follows that its composition $\mathcal{I}^{*} d^{p}$ with the closed operator $d^{p}$ is closed. In the general case suppose $1 \leq p^{\prime}<p$ and $\left\{f_{j}\right\}_{j=1}^{\infty}$ is a sequence in $\mathcal{D o m}\left(d^{p}\right)$ with $f_{j} \rightarrow 0$ in $L^{p}\left(\mathcal{C}_{x_{0}} M ; \mathbf{R}\right)$ and $\mathcal{I}^{*}\left(d^{p} f_{j}\right) \rightarrow \theta$ in $L^{p^{\prime}}\left(\mathcal{C}_{0} \mathbf{R}^{m} ; H^{*}\right)$, some $\theta$. By Theorem 3.4(b) we know $\theta=\mathcal{I}^{*}(\alpha)$ for some $\alpha \in L^{p^{\prime}} \Gamma \mathcal{H}$ 
with $d^{p} f_{j} \rightarrow \alpha$ in $L^{p^{\prime}}$. Since $f_{j} \rightarrow 0$ in $L^{p^{\prime}}, d^{p^{\prime}}$ is closed and $\mathcal{D o m}\left(d^{p}\right) \subset \mathcal{D o m}\left(d^{p^{\prime}}\right)$, we have

$$
\alpha=\lim _{j \rightarrow \infty} d^{p} f_{j}=\lim _{j} d^{p^{\prime}} f_{j}=0 .
$$

Thus $\theta=0$

As observed in the proof of closability, if $f \in \mathcal{D} \operatorname{om}\left(d_{\mathcal{H}}\right)$ then $\mathcal{I}^{*}(f) \in \mathcal{D o m}\left(d^{q}\right)$ all $1 \leq q<\infty$ and

$$
\mathcal{I}^{*}\left(d_{\mathcal{H}} f\right)=d^{q}\left(\mathcal{I}^{*} f\right), \quad f \in \mathcal{D} \operatorname{om}\left(d_{\mathcal{H}}\right)
$$

From this and using (i), (ii)

$$
\left(\mathcal{I}^{*} d_{\mathcal{H}}\right)^{p, p^{\prime}}=\left(\left.d^{p^{\prime}} \mathcal{I}^{*}\right|_{\mathcal{D o m}\left(d_{\mathcal{H}}\right)}\right)^{p, p^{\prime}} \subset d^{p^{\prime}} \mathcal{I}^{*}
$$

where ()$^{p, p^{\prime}}$ indicates the closure as an operator from $L^{p}\left(\mathcal{C}_{x_{0}} M ; \mathbf{R}\right)$ to $L^{p^{\prime}}\left(\mathcal{C}_{0} \mathbf{R}^{m} ; \mathbf{R}\right)$.

The inclusion (4.2) will follow if we show

$$
\left(\mathcal{I}^{*} d_{\mathcal{H}}\right)^{p, p^{\prime}}=\left(\mathcal{I}^{*} d^{p}\right)^{p, p^{\prime}}
$$

This is clear since if $f \in \mathcal{D} \operatorname{om}\left(\mathcal{I}^{*} d^{p}\right) \subset \mathcal{D} \operatorname{om}\left(d^{p}\right)$ there exist $f_{j} \in \mathcal{D} \operatorname{om}\left(d_{\mathcal{H}}\right)$ with $f_{j} \rightarrow f$ in $L^{p}$ and $d_{\mathcal{H}} f_{j} \rightarrow d^{p} f$ in $L^{p}$, but by continuity of $\mathcal{I}^{*}$ this implies that $f \in \mathcal{D} \operatorname{om}\left(\left(\mathcal{I}^{*} d_{\mathcal{H}}\right)^{p, p^{\prime}}\right)$.

Let $\mathbb{D}^{p, 1}\left(\mathcal{C}_{x_{0}} M ; \mathbf{R}\right), \mathbb{D}^{p, 1}\left(\mathcal{C}_{x_{0}} M\right)$ or $\mathbb{D}^{p, 1}$ denote the domain of $d^{p}$ with its graph norm

$$
\|f\|_{\mathbb{D}^{p, 1}}=\left(\left\|d^{p} f\right\|_{L^{p}}^{p}+|f|_{L^{p}}^{p}\right)^{\frac{1}{p}} .
$$

Note that these spaces depend on the choice of $\operatorname{Cyl} \subset \mathcal{D}$ om $\left(d_{\mathcal{H}}\right) \subset B C^{2}$. But see $\S 7.1$.

The boundedness of $\mathcal{I}^{*}$ on $\mathbb{D}^{2,1}$ in the next corollary was known from AidaElworthy [2] for gradient Brownian stochastic differential equations.

Corollary 4.3 The pull back $\mathcal{I}^{*}$ determines a continuous linear map

$$
\mathcal{I}^{*}: \mathbb{D}^{p, 1}\left(\mathcal{C}_{x_{0}} M ; \mathbf{R}\right) \longrightarrow \mathbb{D}^{p^{\prime}, 1}\left(\mathcal{C}_{0} \mathbf{R}^{m} ; \mathbf{R}\right),
$$

for $1<p^{\prime}<p<\infty$, with the property that

$$
\|f\|_{\mathbb{D}^{p, 1}} \leq\left\|\mathcal{I}^{*}(f)\right\|_{\mathbb{D}^{p, 1}}, \quad \text { for } f \in \cup_{q>1} \mathbb{D}^{q, 1} .
$$

If condition $\left(M_{0}\right)$ holds we can take $1 \leq p^{\prime}=p<\infty$ and then the map $\mathcal{I}^{*}$ has closed range in the case of $p=p^{\prime}$, and (4.3) holds for $f \in \mathbb{D}^{1,1}$.

Proof From the theorem $\mathcal{D} \operatorname{om}\left(d^{p}\right) \subset \mathcal{D} \operatorname{om}\left(d^{p^{\prime}} \mathcal{I}^{*}\right)$. A comparison result of Hörmander, (see Yosida [47], Theorem 2, $\S 6$ of chapter II, p79) therefore implies that there is a constant $C_{p, p^{\prime}}$ with

$$
\mid d^{p^{\prime}} \mathcal{I}^{*} f\left\|_{L^{p^{\prime}}} \leq C_{p, p^{\prime}}\right\| f \|_{p, 1}
$$

(Alternatively use Theorem 3.4.) This, plus the continuity of $\mathcal{I}^{*}$ on $L^{p}$, gives the required continuity of $\mathcal{I}^{*}$ on $\mathbb{D}^{p, 1}\left(\mathcal{C}_{x_{0}} M ; \mathbf{R}\right)$ into $\mathbb{D}^{p^{\prime}, 1}\left(\mathcal{C}_{0} \mathbf{R}^{m} ; \mathbf{R}\right)$.

Inequality (4.3) holds by (3.11) and the intertwining (4.2), and implies that $\mathcal{I}^{*}$ has closed range when $p=p^{\prime}$ and Condition $\left(M_{0}\right)$ holds. 
Remark 4.4 (1) As we see in Theorem 6.9 below, an outstanding question is whether $\mathcal{I}^{*}\left[\mathbb{D}^{p, 1}\left(\mathcal{C}_{x_{0}} M ; \mathbf{R}\right)\right]=\mathbb{D}_{\mathcal{F}^{x_{0}}}^{p, 1}\left(\mathcal{C}_{0} \mathbf{R}^{m} ; \mathbf{R}\right)$, the space of $\mathcal{F}^{x_{0}}$ measurable elements of $\mathbb{D}^{p, 1}\left(\mathcal{C}_{0} \mathbf{R}^{m} ; \mathbf{R}\right)$. This appear to be unknown even for gradient Brownian motion systems, with Levi-Civita connection, on any space with curvature (e.g. $S^{n}$ any $n$ ).

(2) Even if Condition $\left(M_{0}\right)$ does not hold we see that for $1<q \leq p<\infty$,

$$
\mathcal{I}^{*}\left[\mathbb{D}^{q, 1}\left(\mathcal{C}_{x_{0}} M ; \mathbf{R}\right)\right] \cap \mathbb{D}^{p, 1}\left(\mathcal{C}_{0} \mathbf{R}^{m} ; \mathbf{R}\right)
$$

is closed in $\mathbb{D}^{p, 1}\left(\mathcal{C}_{0} \mathbf{R}^{m} ; \mathbf{R}\right)$. However, even given Condition $\left(M_{0}\right)$, we do not know whether $\mathcal{I}^{*}(f) \in \mathbb{D}^{p, 1}\left(\mathcal{C}_{0} \mathbf{R}^{m} ; \mathbf{R}\right)$ and $f \in \mathbb{D}^{q, 1}\left(\mathcal{C}_{x_{0}} M ; \mathbf{R}\right)$, some $1<q<$ $p<\infty$, imply that $f \in \mathbb{D}^{p, 1}\left(\mathcal{C}_{x_{0}} M ; \mathbf{R}\right)$. See section 6.1 below.

\section{The divergence operator and the spaces $\mathbb{D}^{p, 1} \mathcal{H}$}

\subsection{The divergence operator div}

From now on we shall take $\operatorname{Dom}\left(d_{\mathcal{H}}\right)$ to be closed under multiplication by elements of $\mathrm{Cyl}$, the set of smooth cylindrical functions on the path space. Assume $1<p<\infty$ and $\frac{1}{p}+\frac{1}{q}=1$. Define

$$
\operatorname{div} \equiv \operatorname{div}^{p}: \operatorname{Dom}\left(\operatorname{div}^{p}\right) \subset L^{p} \Gamma \mathcal{H} \longrightarrow L^{p}\left(\mathcal{C}_{x_{0}} M ; \mathbf{R}\right)
$$

to be the cojoint of $-d^{q}$. That is $V \in \operatorname{Dom}\left(\operatorname{div}^{p}\right)$ if and only $V^{\#}$ is in $\operatorname{Dom}\left(d^{*}\right)$ and $\operatorname{div}^{p} V:=-d^{*}\left(V^{\#}\right)$. So $V \in \operatorname{Dom}\left(\operatorname{div}^{p}\right)$ if and only if $V$ is in $L^{p}$ and there is a constant $C(V)$ such that,

$$
\left|\int d f(V) d \mu_{x_{0}}\right|_{L^{p}} \leq C(V) \cdot|f|_{L^{p}}, \quad \forall f \in \mathbb{D}^{q, 1}\left(\mathcal{C}_{x_{0}} M ; \mathbf{R}\right) .
$$

If Condition $\left(M_{0}\right)$ holds we can take $p=1$ and $q=\infty$. If it is necessary to distinguish the underlying path spaces for the divergence operator, we shall use $\operatorname{Dom}_{\mathcal{C}_{x_{0}} M}\left(\operatorname{div}^{p}\right)$ or $\operatorname{Dom}_{\Omega}\left(\operatorname{div}^{p}\right)$.

Define $\mathbb{Y}: \mathcal{H} \rightarrow H$ whose restriction to $\mathcal{H}_{\sigma}, \sigma \in \mathcal{C}_{x_{0}} M$, is given by

$$
\mathbb{Y}_{\sigma}(h)(\cdot)=\int_{0}^{\cdot} Y_{\sigma_{s}}\left(\frac{\mathbb{D}}{d s} h_{s}\right) d s, \quad h \in \mathcal{H}_{\sigma}^{1} .
$$

Then $\overline{T \mathcal{I}}_{\sigma}\left(\mathbb{Y}_{\sigma}\right): H \rightarrow H$ is the identity map.

For any $h: \mathcal{C}_{x_{0}} M \rightarrow H$ define $\mathcal{K}^{\perp} h: \mathcal{C}_{x_{0}} M \rightarrow H$ by

$$
\left(\mathcal{K}^{\perp} h\right)(\sigma)_{t}=\int_{0}^{t} K^{\perp}\left(\sigma_{s}\right) \dot{h}(\sigma)_{s} d s, \quad 0 \leq t \leq T
$$

and for $h: \mathcal{C}_{0} \mathbf{R}^{m} \rightarrow H$ write

$$
\left(\mathcal{K}^{\perp} h\right)(\omega)_{t}=\int_{0}^{t} \mathcal{K}^{\perp}\left(x_{s}(\omega)\right) \dot{h}(\omega)_{s} d s, \quad 0 \leq t \leq T .
$$

Note that for all $h: \mathcal{C}_{0} \mathbf{R}^{m} \rightarrow H$,

$$
\overline{T \mathcal{I}}\left(\mathcal{K}^{\perp} h\right)=\overline{T \mathcal{I}}(h), \quad \overline{T \mathcal{I}\left(\mathcal{K}^{\perp} h\right)}=\overline{T \mathcal{I}(h)} .
$$


Proposition 5.1 Let $1<p<\infty$. For any $h \in \operatorname{Dom}\left(\operatorname{div}^{p}\right)$ on the Wiener space, and any $0<\delta<p-1, \overline{T \mathcal{I}(h)} \in \operatorname{Dom}\left(\operatorname{div}^{p-\delta}\right)$ on the path manifold. Furthermore

$$
\operatorname{div}(\overline{T \mathcal{I}(h)})=\overline{\operatorname{div} h}=\overline{-\int_{0}^{T}\left\langle\dot{h}_{s}, d B_{s}\right\rangle} .
$$

If condition $\left(M_{0}\right)$ holds we take $\delta=0$ and also allow $p=\infty$.

Proof Take $h \in \operatorname{Dom}\left(\operatorname{div}^{p}\right) \in L^{p}\left(\mathcal{C}_{0} \mathbf{R}^{m} ; H\right)$ then by Corollary 4.3, for $\epsilon>0$,

$$
\int d\left(\mathcal{I}^{*} f\right)(h) d P=-\int \mathcal{I}^{*} f(\operatorname{div} h) d P, \quad \forall f \in \mathbb{D}^{q+\epsilon, 1}\left(\mathcal{C}_{x_{0}} M\right) .
$$

This implies that for all $f \in \mathbb{D}^{q+\epsilon, 1}\left(\mathcal{C}_{x_{0}} M\right)$,

$$
\int d f(\overline{T \mathcal{I}(h)}) d \mu_{x_{0}}=\int \mathcal{I}^{*}(d f)(h) d P=-\int \mathcal{I}^{*} f(\operatorname{div} h) d P=-\int f(\overline{\operatorname{div} h}) d \mu_{x_{0}}
$$

and so for all $h \in \operatorname{Dom}\left(\operatorname{div}^{p}\right)$,

$$
\operatorname{div}(\overline{T \mathcal{I}(h)})(\sigma)=\overline{\operatorname{div} h}(\sigma)
$$

and (5.4) holds since $\operatorname{div} h=-\int_{0}^{T}\left\langle\dot{h}_{s}, d B_{s}\right\rangle$, the Skorohod integral.

Corollary 5.2 Let $1<p^{\prime}<p<\infty$. An $\mathcal{H}$-vector field $V$ is in the domain of $\operatorname{div}^{p^{\prime}}$ if it is in $L^{p^{\prime}}$ and the vector field $h$ on $\mathcal{C}_{0} \mathbf{R}^{m}$ given by

$$
h:=(\mathbb{Y} V) \circ \mathcal{I}: \mathcal{C}_{0} \mathbf{R}^{m} \rightarrow H
$$

is such that $\dot{h}$ is Skorohod integrable, i.e. $h \in \operatorname{Dom}\left(\operatorname{div}^{p}\right)$ for $\mathcal{C}_{0} \mathbf{R}^{m}$. If so

$$
(\operatorname{div} V)(x .)=-\mathbf{E}\left\{\int_{0}^{T}\left\langle\frac{\mathbb{D}}{d s} V(x .), X\left(x_{s}\right) d B_{s}\right\rangle_{x_{s}} \mid \mathcal{F}^{x_{0}}\right\}
$$

where the right hand side is interpreted as the Skorohod integral. In this case

$$
\operatorname{div}(V)=\overline{\operatorname{div}\left(\mathcal{I}^{*}(\mathbb{Y}(V))\right)} .
$$

If condition $\left(M_{0}\right)$ holds we can take $1<p=p^{\prime} \leq \infty$.

Let $\mathbb{D}_{\mathcal{F}^{x_{0}}}^{p, 1}$ be the closed subspace of $\mathbb{D}^{p, 1}(\Omega ; \mathbf{R})$ consisting of $\mathcal{F}^{x_{0}}$ measurable functions.

Corollary 5.3 If $g \in \mathbb{D}_{\mathcal{F}^{x_{0}}}^{p, 1}$ then $d g(-)=d g\left(\mathcal{K}^{\perp}-\right)$ almost surely.

Proof Take $h \in H$. Then $\mathcal{K}^{\perp} h \in \mathbb{D}^{q, 1}$, a subset of Dom(div) by Kree-Kree [32]. Since by Proposition $5.1 \overline{\operatorname{div} h}=\operatorname{div}(\overline{T \mathcal{I}(h)})=\operatorname{div}\left(\overline{T \mathcal{I}\left(\mathcal{K}^{\perp} h\right)}\right)$,

$$
\begin{aligned}
\mathbf{E} d g(h) & =-\mathbf{E} g \operatorname{div} h=-\mathbf{E}\left(g \mathbf{E}\left\{\operatorname{div} h \mid \mathcal{F}^{x_{0}}\right\}\right) \\
& =-\mathbf{E}(g \operatorname{div}(\overline{T \mathcal{I}(h)}) \circ \mathcal{I})=-\mathbf{E}\left(g \operatorname{div}\left(\overline{T \mathcal{I}\left(\mathcal{K}^{\perp} h\right)}\right) \circ \mathcal{I}\right) \\
& =-\mathbf{E}\left(g \operatorname{div}\left(\mathcal{K}^{\perp} h\right)\right)=\mathbf{E} d g\left(\mathcal{K}^{\perp} h\right) .
\end{aligned}
$$

Replace $h$ by $\lambda h$ where $\lambda \in$ Cyl to conclude that almost surely $d g(h)=d g\left(\mathcal{K}^{\perp} h\right)$.

Finally we observe the following version of Corollary 5.2: 
Proposition 5.4 For an $L^{q} \mathcal{H}$-1-form $\phi$, if $\mathbf{E}\left\{\mathcal{I}^{*} \phi \mid \mathcal{F}^{x_{0}}\right\} \in \operatorname{Dom}_{\Omega}\left(d^{*}\right)$ then $\phi$ belongs to $\operatorname{Dom}_{\mathcal{C}_{x_{0}} M}\left(d^{*}\right)$ and

$$
\left(d^{*} \phi\right)_{\sigma}=\mathbf{E}\left\{d^{*} \mathbf{E}\left\{\mathcal{I}^{*} \phi \mid \mathcal{F}^{x_{0}}\right\} \mid \mathcal{I}=\sigma\right\}=\overline{d^{*}\left[\phi_{\mathcal{I}}\left(\overline{T \mathcal{I}}_{\mathcal{I}}(-)\right)\right]}(\sigma) .
$$

Proof Just note that for $f \in \mathrm{Cyl}$,

$$
\begin{aligned}
\int\left\langle d\left(\mathcal{I}^{*} f\right), \mathbf{E}\left\{\mathcal{I}^{*} \phi \mid \mathcal{F}^{x_{0}}\right\}\right\rangle d P & =\int\left\langle\mathcal{I}^{*} f, \mathbf{E}\left\{d^{*} \mathbf{E}\left\{\mathcal{I}^{*} \phi \mid \mathcal{F}^{x_{0}}\right\} \mid \mathcal{F}^{x_{0}}\right\}\right\rangle d P \\
& =\int f(\sigma) \mathbf{E}\left\{d^{*} \mathbf{E}\left\{\mathcal{I}^{*} \phi \mid \mathcal{F}^{x_{0}}\right\} \mid \mathcal{I}=\sigma\right\} d \mu_{x_{0}}(\sigma)
\end{aligned}
$$

on one hand and, since $\mathbf{E}\left\{\mathcal{I}^{*}(-) \mid \mathcal{F}^{x_{0}}\right\}$ is an isometry on one forms,

$$
\begin{aligned}
\int\left\langle d\left(\mathcal{I}^{*} f\right), \mathbf{E}\left\{\mathcal{I}^{*} \phi \mid \mathcal{F}^{x_{0}}\right\}\right\rangle d \mu & \left.=\int\left\langle\mathbf{E}\left\{\mathcal{I}^{*}(d f) \mid \mathcal{F}^{x_{0}}\right\}, \mathbf{E}\left\{\mathcal{I}^{*} \phi \mid \mathcal{F}^{x_{0}}\right\}\right)\right\rangle d P \\
& =\int\langle d f, \phi\rangle d \mu_{x_{0}}
\end{aligned}
$$

on the other hand.

\subsection{Hilbert space valued $L^{p}$ functions}

Let $G$ be a separable Hilbert space and $B=\mathcal{H}$ or $\mathcal{H}^{*}$, or a similar 'tensor bundle'. Denote by $L^{p} \Gamma B$ and $L^{p} \Gamma(G \otimes B)$ respectively the $L^{p}$ sections of $B$ and those of the tensor product of the 'bundle' $B$ with the trivial $G$ bundle over $\mathcal{C}_{x_{0}} M$. We always use $\otimes$ to refer Hilbert space completions and $\otimes_{0}$ the incomplete algebraic tensor products. For each densely defined linear map $T$ from $L^{p}\left(\mathcal{C}_{x_{0}} M ; \mathbf{R}\right)$ to $L^{p} \Gamma B$ there is a naturally defined linear operator $T^{G} \equiv \operatorname{Id} \otimes T$ from $L^{p}\left(\mathcal{C}_{x_{0}} M ; G\right)$ to $L^{p} \Gamma(G \otimes B)$ with domain $\operatorname{Dom}\left(T^{G}\right) \equiv \operatorname{Dom}(T) \otimes_{0} G$, namely

$\operatorname{Dom}\left(T^{G}\right)=\left\{F: \mathcal{C}_{x_{0}} M \rightarrow G \mid F(\sigma)=\sum_{j=1}^{n} f_{j}(\sigma) g_{j}, g_{j} \in G, f_{j} \in \operatorname{Dom}(T), n \in \mathbb{N}\right\}$

and such that $T^{G}(f \otimes g)=T(f) \otimes g$ for $f \in \operatorname{Dom}(T)$ and $g \in G$.

Proposition 5.5 If $T$ with $\operatorname{Dom}(T)$ is a closable operator then so is $T^{G} \equiv \operatorname{Id} \otimes T$ with $\operatorname{Dom}\left(T^{G}\right)=G \otimes_{0} \operatorname{Dom}(T)$.

Proof Take $F_{n} \in \operatorname{Dom}\left(T^{G}\right)$ converging to 0 in $L^{p}\left(\mathcal{C}_{x_{0}} M ; G\right)$ with $T F_{n} \rightarrow \alpha$. For an orthonomal basis $\left\{e_{j}\right\}$ for $G$ write $F_{n}(\sigma)=\sum_{j=1}^{\infty} f_{n}^{j}(\sigma) e_{j}$ and $\alpha=\sum_{j} e_{j} \otimes \alpha_{j}$. Then $T f_{n}^{j} \rightarrow \alpha_{j}$ and $f_{n}^{j} \rightarrow 0$ in $L^{p}$ as $n \rightarrow \infty$. Consequently $\alpha_{j}=0$.

Now take $T$ to be $d_{\mathcal{H}}$ with $\operatorname{Dom}\left(d_{\mathcal{H}}\right)$ the set of smooth cylindrical functions. Define

$$
d \equiv d^{p} \equiv d^{p, G}: \operatorname{Dom}\left(d^{p, G}\right) \subset L^{p}\left(\mathcal{C}_{x_{0}} M ; G\right) \rightarrow L^{p} \Gamma\left(G \otimes \mathcal{H}^{*}\right) \sim L^{p} \Gamma\left(\mathbb{L}_{2}(\mathcal{H} ; G)\right)
$$

to be the closure of $\left(d_{\mathcal{H}}\right)^{G}$ which exists by Proposition 5.5. Its domain shall be denoted by $\mathbb{D}^{p, 1}\left(\mathcal{C}_{x_{0}} M ; G\right)$ and is the closure of $\operatorname{Dom}(d) \equiv G \otimes_{0} \operatorname{Dom}\left(d_{\mathcal{H}}\right)$ under the graph norm.

The following elementary lemma is useful in section 5.4. 
Lemma 5.6 Suppose $1<p<\infty$, allowing $p=1$ if Condition $\left(M_{0}\right)$ holds. Let $G_{1}$ and $G_{2}$ be two Hilbert spaces and $\theta: M \rightarrow \mathbb{L}\left(G_{1} ; G_{2}\right)$ a $C^{1}$ map. Suppose $f: C_{x_{0}} M \rightarrow L^{2}\left([0, T] ; G_{1}\right)$ is in $\mathbb{D}^{p, 1}\left(\mathcal{C}_{x_{0}} M ; L^{2}\left([0, T] ; G_{1}\right)\right)$. Then

$$
\Theta(f): C_{x_{0}} M \rightarrow L^{2}\left([0, T] ; G_{2}\right)
$$

given by $\sigma \mapsto\left[t \mapsto \theta\left(\sigma_{t}\right)\left(f(\sigma)_{t}\right)\right.$ is in $\mathbb{D}^{p, 1}\left(\mathcal{C}_{x_{0}} M ; L^{2}\left([0, T] ; G_{2}\right)\right)$ with

$$
\left[d^{p}(\Theta(f))_{\sigma}(v)\right]_{t}=(d \theta)_{\sigma_{t}}\left(v_{t}\right)\left(f(\sigma)_{t}\right)+\theta\left(\sigma_{t}\right)\left(d^{p} f_{\sigma}(v)\right)_{t}
$$

Proof Take $f_{n} \rightarrow f$ in $\mathbb{D}^{p, 1}\left(\mathcal{C}_{x_{0}} M ; L^{2}\left([0, T] ; G_{1}\right)\right)$ where

$$
f_{n}(\sigma)=\sum_{j=1}^{k_{n}} \lambda_{j}^{n}(\sigma) h^{j}
$$

with $\lambda_{j}^{n}$ real valued smooth cylindrical functions and $h^{j} \in L^{2}\left([0, T] ; G_{1}\right)$. Then

$$
\Theta\left(f_{n}\right)(\sigma)(t)=\sum_{j=1}^{k_{n}} \lambda_{j}^{n}(\sigma) \theta\left(\sigma_{t}\right) h_{t}^{j}
$$

Clearly $\Theta\left(f_{n}\right) \rightarrow \Theta(f)$ in $L^{p}\left(\mathcal{C}_{x_{0}} M ; L^{2}\left([0, T] ; G_{2}\right)\right)$ and $\Theta\left(f_{n}\right)$ is Fréchet differentiable as a map into $L^{2}\left([0, T] ; G_{2}\right)$. For $v \in \mathcal{H}_{\sigma}$,

$$
\begin{aligned}
{\left[d_{\mathcal{H}}\left(\Theta\left(f_{n}\right)\right)_{\sigma}(v)\right]_{t} } & =\sum_{j=1}^{k_{n}}\left(d_{\mathcal{H}} \lambda_{j}^{n}\right)_{\sigma}(v) \theta\left(\sigma_{t}\right) h_{t}^{j}+\sum_{j=1}^{k_{n}} \lambda_{j}^{n}(\sigma)(d \theta)_{\sigma_{t}}\left(v_{t}\right) h_{t}^{j} \\
& \left.=(d \theta)_{\sigma_{t}}\left(v_{t}\right)\left(f_{n}(\sigma)_{t}\right)+\theta\left(\sigma_{t}\right)\left(d f_{n}\right)_{\sigma}(v)_{t}\right)
\end{aligned}
$$

and we see that $d_{\mathcal{H}}\left(\Theta\left(f_{n}\right)\right)$ converges in $L^{p}\left(\mathcal{C}_{x_{0}} M ; L^{2}\left([0, T] ; G_{2}\right)\right)$ with limit given by the right hand side of (5.6). Finally just approximate $\theta\left(\sigma_{t}\right)$ by a sequence of functions $\theta_{n} \in \mathbb{D}^{p, 1}\left(L^{2}\left([0, T] ; G_{2}\right)\right)$, for example set

$$
\theta_{n}(\sigma)_{t}=\frac{t-t_{j}^{n}}{t_{j+1}^{n}-t_{j}^{n}} \theta\left(\sigma\left(t_{j+1}^{n}\right)\right)+\theta\left(\sigma\left(t_{j}^{n}\right)\right), t_{j}^{n} \leq t<t_{j+1}^{n}
$$

for suitable partitions $0 \leq t_{1}^{n} \leq \ldots \leq t_{j}^{n} \leq \ldots \leq T$.

\subsection{Pull back of Hilbert space valued functions and $H$-forms}

We follow the notation of section 5.2. For $p=2$ there is the canonical isometry of $L^{2}\left(\mathcal{C}_{x_{0}} M ; \mathbf{R}\right) \otimes G$ with $L^{2}\left(\mathcal{C}_{x_{0}} M ; G\right)$ mapping $\operatorname{Dom}(d) \otimes G$ onto $\operatorname{Dom}\left(d^{G}\right)$.

By a $G$-valued $\mathcal{H}$-1-form $\phi$ on $\mathcal{C}_{x_{0}} M$ we mean a measurable section of the bundle $\mathbb{L}_{2}(\mathcal{H} ; G)$, (or equivalence class of such sections under almost sure equality). There is the standard identification of $\mathbb{L}_{2}\left(\mathcal{H}_{\sigma} ; G\right)$ with $G \otimes \mathcal{H}_{\sigma}$. It is given by $(g \otimes h)(v)=$ $\langle h, v\rangle_{\mathcal{H}_{\sigma}} g$. We shall use $\phi^{\#}$ to denote the section of $G \otimes \mathcal{H}$ corresponding to a $G$ valued $\mathcal{H}$-1-form $\phi$. Note that we can differentiate such $\phi^{\#}$ to obtain $\left(1_{G} \otimes \frac{\mathbb{D}}{d s}\right) \phi_{\sigma, s}^{\#} \in$ $G \otimes T_{\sigma(s)} M, 0 \leq s \leq T, \sigma \in \mathcal{C}_{x_{0}} M$.

If $f: \mathcal{C}_{x_{0}} M \rightarrow G$ is in $\mathbb{D}^{p, 1}$, its differential $(d f)_{\sigma} \in \mathbb{L}_{2}(\mathcal{H} ; G)$ then determines the gradient $\nabla f \in G \otimes \mathcal{H}$ by $\nabla f(\sigma)=(d f)_{\sigma}^{\#}$. In the Nualart-Pardoux notation we obtain $\left(s \mapsto D_{s} f\right) \in \Gamma\left(G \otimes L^{2} \mathcal{E}\right)$ by the isometries

$$
\mathbb{L}_{2}(\mathcal{H} ; G) \sim G \otimes \mathcal{H}^{1 \otimes \frac{\mathrm{D}}{d s}} G \otimes L^{2} \mathcal{E} .
$$


Theorem 5.7 Theorem 3.4 and Theorem 4.2 and Corollary 4.3 hold for $G$-valued functions. For a measurable $G$-valued $\mathcal{H}$-1-form $\phi$ in $L^{p}$ the pull back $\mathcal{I}^{*}(\phi): \mathcal{C}_{0} \mathbf{R}^{m} \rightarrow$ $\mathbb{L}_{2}(H ; G)$ is given by, for $h \in H$,

$$
\mathcal{I}^{*}(\phi)(h)=\int_{0}^{T}\left[\left(1_{G} \otimes \frac{\mathbb{D}}{d s}\right) \phi_{s}^{\#}\right]^{\#}\left(\nabla_{T \mathcal{I}_{s}(h)} X\left(\tilde{/}_{s} d \beta_{s}\right)+X\left(x_{s}\right)\left(\dot{h}_{s}\right) d s\right) .
$$

Proof The proofs are essentially the same as those for the real valued case. Here we assume that $p=2$ to demonstrate the proof. If the $G$-valued $\phi$ has $\phi^{\#}=\sum_{j=1}^{k} g_{j} \otimes \psi_{j}^{\#}$ where $\psi_{j}^{\#} \in \Gamma \mathcal{H}$ and $g_{j} \in G$ for $j=1$ to $k$, we immediately obtain (5.7) from (3.8). The general case and the rest of Theorem 3.4 follow by taking limits, in particular by observing that (3.18) remains true in the $G$-valued case. The proof of Theorem 4.2 is based on (3.18) and so is easily seen to extend to the $G$-valued case. The crucial remark is that the stochastic integral in (5.7) can be considered as an $\mathbb{L}_{2}(H ; G)$-valued integral applied to $h$. To see this and perform the necessary estimates we need to show

$$
\int_{0}^{T}\left\|\left[\left(1_{G} \otimes \frac{\mathbb{D}}{d s}\right) \phi_{s}^{\#}\right]^{\#} \nabla_{T \mathcal{I}_{s}(-)} X^{j}\right\|_{\mathbb{L}_{2}(H, G)}^{2} d s
$$

is finite almost surely for $j=1, \ldots, m$. First noted that the assumption that $\phi_{\sigma}$ : $\mathcal{H} \rightarrow G$ is in $L_{2}$ implies that so is $V \mapsto \phi_{\sigma}(\mathbf{W} . V)$ from $L^{2} \mathcal{E}$ to $G$, where $L^{2} \mathcal{E}$ is the Hilbert bundle of $E$ valued $L^{2}$ tangent vector fields on $\mathcal{C}_{x_{0}} M$. Moreover

$$
\phi_{\sigma}(\mathbf{W} . V)=\int_{0}^{T}\left\langle\left(1_{G} \otimes \frac{\mathbb{D}}{d s}\right) \phi_{s}^{\#}, V_{s}\right\rangle_{\sigma(s)} d s .
$$

The norm of $\phi_{\sigma}(\mathbf{W} .-)$ equals $\left\|\phi_{\sigma}\right\|_{\mathbb{L}_{2}\left(\mathcal{H}_{\sigma} ; G\right)}^{2}$ and is given by

$$
\left\|\phi_{\sigma} \circ \mathbf{W}\right\|_{\mathbb{L}_{2}\left(\mathbb{L}^{2} \mathcal{E} ; G\right)}^{2}=\int_{0}^{T}\left\|\left(1_{G} \otimes \frac{\mathbb{D}}{d s}\right) \phi_{s}^{\#}\right\|_{\mathbb{L}_{2}\left(E_{\sigma(s)} ; G\right)}^{2} d s,
$$

which is finite. Now for $\sigma=\mathcal{I}(\omega)$,

$$
\begin{aligned}
& \int_{0}^{T}\left\|\left[\left(1_{G} \otimes \frac{\mathbb{D}}{d s}\right) \phi_{s}^{\#}\right]^{\#} \nabla_{T_{\omega} \mathcal{I}_{s}(-)} X^{j}\right\|_{\mathbb{L}_{2}(H ; G)}^{2} d s \\
\leq & \int_{0}^{T}\left\|\left[\left(1_{G} \otimes \frac{\mathbb{D}}{d s}\right) \phi_{s}^{\#}\right]^{\#}\right\|_{\mathbb{L}_{2}\left(E_{\sigma(s)} ; G\right)}^{2}\left\|\nabla_{T_{\omega} \mathcal{I}_{s}(-)} X^{j}\right\|_{\mathbb{L}\left(H ; E_{\sigma(s)}\right)}^{2} d s \\
\leq & \left\|\phi_{\sigma} \circ \mathbf{W}\right\|_{\mathbb{L}_{2}\left(L^{2} \mathcal{E} ; G\right)}^{2} \cdot \sup _{s}\left\|\nabla_{T_{\omega} \mathcal{I}_{s}(-)} X^{j}\right\|_{\mathbb{L}\left(H ; E_{\sigma(s)}\right)}^{2} \\
\leq & \text { constant } \cdot\left\|\phi_{\sigma} \circ \mathbf{W}\right\|_{\mathbb{L}_{2}\left(L^{2} \mathcal{E} ; G\right)}^{2} \sup _{s}\left\|T_{\omega} \mathcal{I}\right\|_{\mathbb{L}\left(H ; T_{\sigma} \mathcal{C}_{x_{0}} M\right)}^{2}<\infty .
\end{aligned}
$$

There are no difficulties with Corollary 4.3. 


\subsection{The space $\mathbb{D}^{p, 1} \mathcal{H}$}

For $1<p<\infty$, and allowing $p=1$ if Condition $\left(M_{0}\right)$ holds, define

$$
\mathbb{D}^{p, 1} \mathcal{H}=\left\{V \in L^{p} \Gamma \mathcal{H} \quad \mid \quad \mathbb{Y} .(V(\cdot)) \in \mathbb{D}^{p, 1}\left(\mathcal{C}_{x_{0}} M ; H\right)\right\}
$$

Equip this space with the obvious norm:

$$
\|V\|_{\mathbb{D}^{p, 1} \mathcal{H}}=\|\mathbb{Y}(V(\cdot))\|_{\mathbb{D}^{p, 1}\left(\mathcal{C}_{x_{0}} M ; H\right)}=\left(\mathbf{E}|\mathbb{Y}(V)|^{p}+\mathbf{E}|d(\mathbb{Y}(V))|^{p}\right)^{\frac{1}{p}} .
$$

This depends only on the connection on $E$ not on the specific stochastic differential equation (2.5), or equivalently not on the particular choice of $X$ as can be easily seen by Lemma 5.6. In fact $\mathbb{D}^{p, 1} \mathcal{H}$ can also be described by covariant differentiation, see $\S 8.3$ below. Similarly we can define $\mathbb{D}^{p, 1} \mathcal{H}^{*}$ :

$$
\mathbb{D}^{p, 1} \mathcal{H}^{*}=\left\{\phi \in L^{p} \Gamma \mathcal{H}^{*} \quad \mid \quad \phi(\boldsymbol{X}(-)) \in \mathbb{D}^{p, 1}\left(\mathcal{C}_{x_{0}} M ; H^{*}\right)\right\} .
$$
$\mathcal{C}_{0} \mathbf{R}^{m}$

We have the following analogue of a fundamental result of Kree-Kree [32] for

Theorem 5.8 For $1<p^{\prime}<p<\infty$, the set $\mathbb{D}^{p, 1} \mathcal{H}$ is contained in Dom(div $\left.{ }^{p^{\prime}}\right)$ and $\operatorname{div}^{p^{\prime}}: \mathbb{D}^{p, 1} \mathcal{H} \rightarrow L^{p^{\prime}}\left(\mathcal{C}_{x_{0}} M ; \mathbf{R}\right)$ is continuous. If Condition $\left(M_{0}\right)$ holds we may take $p=p^{\prime}$

Proof Take $r$ with $p^{\prime}<r<p$. If $V \in \mathbb{D}^{p, 1} \mathcal{H}$ then $\mathbb{Y}(V) \circ \mathcal{I} \in \mathbb{D}^{r, 1}(\Omega ; H) \subset$ $\operatorname{Dom}_{\Omega}\left(\operatorname{div}^{r}\right)$, by Theorem 5.7 and the corresponding result for Wiener space of KreeKree [32]. Then $V=\overline{T \mathcal{I}(\mathbb{Y}(V))} \in \operatorname{Dom}_{\mathcal{C}_{x_{0}} M}\left(\operatorname{div}^{p^{\prime}}\right)$ by Proposition 5.1. Finally note that

$$
\mathbb{D}^{p, 1} \mathcal{H}^{\mathcal{I}^{*}(\boldsymbol{Y}-)} \longrightarrow \mathbb{D}^{r, 1}(\Omega ; H) \stackrel{\text { div }-}{\longrightarrow} L^{p^{\prime}}(\Omega ; \mathbf{R}) \stackrel{\text { conditional expectation }}{\longrightarrow} L^{p^{\prime}}\left(\mathcal{C}_{x_{0}} M ; \mathbf{R}\right)
$$

is a continuous map and by Corollary 5.2, agrees with the divergence operator restricted to $\mathbb{D}^{p, 1} \mathcal{H}$. Note that the continuity also follows from the closed graph theorem.

The following is a compliment of Proposition 5.1:

Proposition 5.9 For $1<p<\infty$, set

$$
U=\left\{h \in \mathbb{D}^{p, 1}(\Omega, H) \quad \mid \quad \overline{T \mathcal{I}(h)} \in \mathbb{D}^{p, 1} \mathcal{H}\right\} .
$$

Then $U$ is total in $\mathbb{D}^{p, 1}(\Omega ; H)$ and thus total in the domain, Dom(div $\left.{ }^{p}\right)$, of the divergence on Wiener space.

Proof Consider the family of functions

$$
U_{1} \equiv\left\{k^{\prime} \exp \left(\int_{0}^{T}\left\langle\dot{k}_{s}, d B_{s}\right\rangle-\frac{1}{2} \int_{0}^{T}\left|\dot{k}_{s}\right|^{2} d s\right) \mid k^{\prime}, k \in H\right\} .
$$

Since the exponential martingales are total in $\mathbb{D}^{p, 1}(\Omega ; \mathbf{R})$ it is clear from the definition that $U_{1}$ is total in $\mathbb{D}^{p, 1}(\Omega ; H)$ and so it is sufficient to show that

$$
\text { Image }\left[\overline{T \mathcal{I}(h)}: h \in U_{1}\right] \subset \mathbb{D}^{p, 1}(\mathcal{H}),
$$


or equivalently that $\mathbb{Y}(\overline{T \mathcal{I}(h)})$ belongs to $\mathbb{D}^{p, 1}\left(\mathcal{C}_{x_{0}} M ; H\right)$. In fact for

$$
h .=k^{\prime} \exp \left(\int_{0}^{T}\left\langle\dot{k}_{s}, d B_{s}\right\rangle-\frac{1}{2} \int_{0}^{T}\left|\dot{k}_{s}\right|^{2} d s\right)
$$

we can write

$$
\begin{aligned}
h .= & k_{\cdot}^{\prime} \exp \left(\int_{0}^{T}\left\langle\dot{k}_{s}, / / s d \breve{B}_{s}\right\rangle-\frac{1}{2} \int_{0}^{T}\left|K^{\perp}\left(x_{s}\right) \dot{k}_{s}\right|^{2} d s\right) \\
& \cdot \exp \left(\int_{0}^{T} \int_{0}^{T}\left\langle\dot{k}_{s}, K\left(x_{s}\right) d B_{s}\right\rangle-\frac{1}{2} \int_{0}^{T}\left|K\left(x_{s}\right) \dot{k}_{s}\right|^{2} d s\right) . \\
\overline{T \mathcal{I}_{t}(h .)}= & \exp \left(\int_{0}^{T}\left\langle\dot{k}_{s}, / /{ }_{s} d \breve{B}_{s}\right\rangle-\frac{1}{2} \int_{0}^{T}\left|K^{\perp}\left(x_{s}\right) \dot{k}_{s}\right|^{2} d s\right) \\
& \times \mathbf{E}\left\{T \mathcal{I}_{t}\left(k^{\prime} \exp \int_{0}^{T}\left\langle\dot{k}_{s}, K\left(x_{s}\right) d B_{s}\right\rangle-\frac{1}{2} \int_{0}^{T}\left|K\left(x_{s}\right) \dot{k}_{s}\right|^{2} d s\right) \mid \mathcal{F}^{x_{0}}\right\} .
\end{aligned}
$$

Set

$$
f_{t}=\mathbf{E}\left\{\exp \left(\int_{0}^{T}\left\langle\dot{k}_{s}, K\left(x_{s}\right) d B_{s}\right\rangle-\frac{1}{2} \int_{0}^{T}\left|K\left(x_{s}\right) \dot{k}_{s}\right|^{2} d s\right) \mid \mathcal{F}^{x_{0}} \vee \mathcal{F}_{t}\right\}
$$

Then $\left(f_{t}, 0 \leq t \leq T\right)$ is a martingale with respect to $\left\{\mathcal{F}^{x_{0}} \vee \mathcal{F}_{t}\right\}$ and so

$$
f_{t}=\exp \left(\int_{0}^{t}\left\langle\dot{k}_{s}, K\left(x_{s}\right) d B_{s}\right\rangle-\frac{1}{2} \int_{0}^{t}\left|K\left(x_{s}\right) \dot{k}_{s}\right|^{2} d s\right),
$$

giving

$$
\begin{aligned}
& \mathbf{E}\left\{T \mathcal{I}_{t}\left(k^{\prime}\right) \exp \left(\int_{0}^{T}\left\langle\dot{k}_{s}, K\left(x_{s}\right) d B_{s}\right\rangle-\frac{1}{2} \int_{0}^{T}\left|K\left(x_{s}\right) \dot{k}_{s}\right|^{2} d s\right) \mid \mathcal{F}^{x_{0}}\right\} \\
= & \mathbf{E}\left\{T \mathcal{I}_{t}\left(k^{\prime}\right) \exp \left(\int_{0}^{t}\left\langle\dot{k}_{s}, K\left(x_{s}\right) d B_{s}\right\rangle-\frac{1}{2} \int_{0}^{t}\left|K\left(x_{s}\right) \dot{k}_{s}\right|^{2} d s\right) \mid \mathcal{F}^{x_{0}}\right\} .
\end{aligned}
$$

On the other hand, if we set

$$
V_{t}=T \mathcal{I}_{t}\left(k^{\prime}\right) \exp \left(\int_{0}^{t}\left\langle\dot{k}_{s}, K\left(x_{s}\right) d B_{s}\right\rangle-\frac{1}{2} \int_{0}^{t}\left|K\left(x_{s}\right) \dot{k}_{s}\right|^{2} d s\right),
$$

then as in Elworthy-LeJan-Li [15], Elworthy-Li [19]

$$
\begin{aligned}
\mathbb{D} V_{t}= & \nabla X\left(V_{t}\right) d B_{t}+\frac{1}{2} \nabla X\left(V_{t}\right)\left(K\left(x_{t}\right) \dot{k}_{t}\right) d t \\
& +T \mathcal{I}_{t}\left(k^{\prime}\right) \exp \left(\int_{0}^{t}\left\langle\dot{k}_{s}, K\left(x_{s}\right) d B_{s}\right\rangle-\frac{1}{2} \int_{0}^{t}\left|K\left(x_{s}\right) \dot{k}_{s}\right|^{2} d s\right)\left\langle\dot{k}_{t}, K\left(x_{t}\right) d B_{t}\right\rangle \\
= & \nabla X\left(V_{t}\right) d B_{t}+\frac{1}{2} \nabla X\left(V_{t}\right)\left(K\left(x_{t}\right) \dot{k}_{t}\right) d t+\left\langle\dot{k}_{t}, K\left(x_{t}\right) d B_{t}\right\rangle V_{t} .
\end{aligned}
$$


Consequently, for $\bar{V}_{t}(\sigma)=\mathbf{E}\left\{V_{t} \mid x .=\sigma\right\}$,

$$
\frac{\mathbb{D} \overline{V_{t}}}{d t} \circ \mathcal{I}=\frac{1}{2} \nabla_{\overline{V_{t}}(x .)} X\left(K\left(x_{t}\right) \dot{k}_{t}\right) d t .
$$

Thus

$$
\frac{d}{d t} \mathbb{Y}\left(\overline{V_{t}}(x .)\right)=Y\left(\frac{\mathbb{D}}{d t} \bar{V}_{t}(x .)\right)=\frac{1}{2} Y\left(\nabla X \left(\overline{T \mathcal{I}_{t}}\left(\mathbb{Y}(\overline{V .(x .)})\left(K\left(x_{t}\right) \dot{k}_{t}\right)\right) .\right.\right.
$$

Since solutions of such a stochastic differential equation are in $\mathbb{D}^{p, 1}$ for all $1<p<\infty$ by standard results, $\mathbb{Y}(\bar{V}.) \in \mathbb{D}^{p, 1}\left(\mathcal{C}_{x_{0}} M ; H\right)$. See Lemma 6.12 below. Finally note that

$$
{\overline{T \mathcal{I}_{t}(h .)}}_{\sigma}=\exp \left(\int_{0}^{T}\left\langle\dot{k}_{s}, / / s d \breve{B}_{s}\right\rangle-\frac{1}{2} \int_{0}^{T}\left|K^{\perp}\left(\sigma_{s}\right) \dot{k}_{s}\right|^{2} d s\right) \overline{V_{t}}(\sigma) .
$$

Since such exponential martingales belong to $\mathbb{D}^{q, 1}$ for all finite $q$ we see that

$$
\sigma \mapsto \mathbb{Y}\left({\overline{T \mathcal{I}_{t}(h .)}}_{\sigma}\right)=\mathbb{Y}\left(\bar{V}_{t}(\sigma)\right) \exp \left(\int_{0}^{T}\left\langle\dot{k}_{s}, / /{ }_{s} d \breve{B}_{s}\right\rangle-\frac{1}{2} \int_{0}^{T}\left|K^{\perp}\left(\sigma_{s}\right) \dot{k}_{s}\right|^{2} d s\right)
$$

belongs to $\mathbb{D}^{p, 1}\left(\mathcal{C}_{x_{0}} M ; H\right)$ for all $1 \leq p<\infty$.

\section{On the Markov Uniqueness of $d$}

Throughout section 6 we take $\operatorname{Dom}\left(d_{\mathcal{H}}\right)=$ Cyl. To define the weak derivatives we shall need to assume $\left.\mathbb{D}^{q, 1} \mathcal{H}^{*} \subset \operatorname{Dom}\left(d^{p}\right)^{*}\right)$, which is guaranteed by Theorem 5.8 if Condition $\left(M_{0}\right)$ holds or if $X$ is injective.

\subsection{Weak Differentiability}

For $\frac{1}{p}+\frac{1}{q}=1$ and $1<p<\infty$, the weak Sobolev space $W^{p, 1}\left(\mathcal{C}_{x_{0}} M\right)$, abbreviated as $W^{p, 1}$, is the domain of the adjoint of the restriction of $\left(d^{p}\right)^{*}$ to $\mathbb{D}^{q, 1} \mathcal{H}^{*}$ :

$$
W^{p, 1}=\operatorname{Dom}\left(\left(\left.\left(d^{p}\right)^{*}\right|_{\mathbb{D}^{q, 1} \mathcal{H}^{*}}\right)^{*}\right)
$$

furnished with its graph norm. More precisely a function $f$ belongs to $W^{p, 1}$ if and only if it is in $L^{p}$ and there is a constant $C(f)$ such that

$$
\left|\int_{\mathcal{C}_{x_{0}} M} f d^{*} \phi d \mu_{x_{0}}\right| \leq C(f)|\phi|_{L^{q}}, \forall \phi \in \mathbb{D}^{q, 1} \mathcal{H}^{*}
$$

Equivalently,

$$
W^{p, 1}=\left\{f \in L^{p}:\left|\int \operatorname{div}(V) f d \mu_{x_{0}}\right| \leq C|V|_{L^{q}}, \text { for all } V \in \mathbb{D}^{q, 1} \mathcal{H}, \text { some } C\right\} .
$$

If $f \in W^{p, 1}$ it has a "weak derivative" $\tilde{d} f \in L^{p} \Gamma \mathcal{H}^{*}$ defined by

$$
\int \tilde{d} f(V(\sigma)) d \mu_{x_{0}}(\sigma)=-\int f(\sigma) \operatorname{div} V(\sigma) d \mu_{x_{0}}(\sigma), \forall V \in \mathbb{D}^{q, 1} \mathcal{H},
$$


and

$$
\tilde{d}=\left(\left.\left(d^{p}\right)^{*}\right|_{\mathbb{D}^{q, 1} \mathcal{H}^{*}}\right)^{*}
$$

as a closed densely defined operator on $L^{p}$. Denote by $\tilde{\nabla}$ the corresponding weak gradient with values in $L^{p} \Gamma \mathcal{H}$. Note that $\tilde{d}$ is an extension of $\left(d, \mathbb{D}^{p, 1}\right)$.

Recall that Cyl denotes the space of smooth cylindrical functions on $\mathcal{C}_{x_{0}} M$. Set

$$
\mathrm{Cyl}^{0} \mathcal{H}^{*}=\text { linear span }\{g d k \mid g, k \in \mathrm{Cyl}\}
$$

and define

$$
{ }^{0} W^{p, 1}=\operatorname{Dom}\left(d^{*} \mid \mathrm{Cyl}^{0} \mathcal{H}^{*}\right)^{*} .
$$

From Proposition 6.14 below, $\mathrm{Cyl}{ }^{0} \mathcal{H}^{*} \subset \mathbb{D}^{q, 1} \mathcal{H}^{*} \subset \operatorname{Dom}\left(d^{*}\right)$ and so

$$
\mathbb{D}^{p, 1} \subseteq W^{p, 1} \subseteq{ }^{0} W^{p, 1} .
$$

In Theorem 6.7 below we show that $W^{p, 1}={ }^{0} W^{p, 1}$.

Theorem 6.1 Suppose Condition $\left(M_{0}\right)$ holds. For $1<p<\infty$, the following are equivalent:

(i) $f \in W^{p, 1}\left(\mathcal{C}_{x_{0}} M ; \mathbf{R}\right)$

(ii) $\mathcal{I}^{*}(f) \in \mathbb{D}^{p, 1}\left(\mathcal{C}_{0} \mathbf{R}^{m} ; \mathbf{R}\right)$

(iii) $f \in W^{r, 1}\left(\mathcal{C}_{x_{0}} M ; \mathbf{R}\right) \cap L^{p}\left(\mathcal{C}_{x_{0}} M ; \mathbf{R}\right)$ some $r \in(1, p)$ and the weak derivative $\tilde{d} f$ is in $L^{p}$.

\section{Moreover}

(iv) there is the following intertwining of $\tilde{d}$ and $\mathcal{I}^{*}$ :

$$
d\left(\mathcal{I}^{*} f\right)=\mathcal{I}^{*}(\tilde{d} f), \text { for all } f \in W^{p, 1}\left(\mathcal{C}_{x_{0}} M ; \mathbf{R}\right) .
$$

(v) if $f \in W^{p, 1}\left(\mathcal{C}_{x_{0}} M ; \mathbf{R}\right)$,

$$
(\tilde{d} f)_{\sigma}=\mathbb{E}\left\{d\left(\mathcal{I}^{*} f\right)_{\omega} \mid x .(\omega)=\sigma\right\} \mathbb{Y}_{\sigma} .
$$

Proof Let $V \in \mathbb{D}^{q, 1} \mathcal{H}$. Suppose $\mathcal{I}^{*}(f) \in \mathbb{D}^{p, 1}$ then by Corollary 5.2 and Kree-Kree [32],

$$
\begin{aligned}
\int_{\mathcal{C}_{x_{0}} M} f \operatorname{div}(V) d \mu_{x_{0}} & =\int_{\mathcal{C}_{0} \mathbf{R}^{m}} \mathcal{I}^{*}(f) \operatorname{div}(V) \circ \mathcal{I} d \mathbb{P} \\
& =\int_{\mathcal{C}_{0} \mathbf{R}^{m}} \mathcal{I}^{*}(f) \operatorname{div} \mathcal{I}^{*}\left(\mathbb{Y}_{-}(V(-))\right) d \mathbb{P} \\
& =-\int_{\mathcal{C}_{0} \mathbf{R}^{m}} d\left(\mathcal{I}^{*}(f)\right)_{\omega}\left(\mathbb{Y}_{x .(\omega)}(V(x .(\omega))) d \mathbb{P}(\omega)\right. \\
& =-\int_{\mathcal{C}_{x_{0}} M} \mathbb{E}\left\{d\left(\mathcal{I}^{*}(f)\right)_{\omega} \mid x .(\omega)=\sigma\right\} \mathbb{Y}_{\sigma}(V(\sigma)) d \mu_{x_{0}}(\sigma) .
\end{aligned}
$$

Thus (ii) implies (i) and (6.5) holds. 
To show (i) implies (ii), suppose $f \in W^{p, 1}\left(\mathcal{C}_{0} \mathbf{R}^{m}\right)$ and take $V=\overline{T \mathcal{I}(h)}$ where $h \in U$, as defined in Proposition 5.9. By definition $V \in \mathbb{D}^{q, 1} \mathcal{H}$ and by Proposition 5.1 $\operatorname{div}(V)=\overline{\operatorname{div}(h)}$. So

$$
\begin{aligned}
\int_{\Omega} \operatorname{div}(h) \mathcal{I}^{*}(f) d P & =\int_{\mathcal{C}_{x_{0}} M} \operatorname{div}(V) f d \mu_{x_{0}} \\
& \leq C(f)\|\overline{T \mathcal{I}(-)}\||h|_{L^{q}}
\end{aligned}
$$

by Corollary 3.7. Since $U$ is total in $\mathbb{D}^{q, 1}(\Omega, H)$ the inequality holds for all $h \in$ $\mathbb{D}^{q, 1}(\Omega ; H)$. Consequently $\mathcal{I}^{*}(f) \in W^{p, 1}(\Omega)=\mathbb{D}^{p, 1}(\Omega ; \mathbf{R})$, using Sugita [45].

Next observe for $f \in W^{p, 1}\left(\mathcal{C}_{x_{0}} M\right), h \in \mathbb{D}^{q, 1}$,

$$
\begin{aligned}
& \int_{\Omega} d\left(\mathcal{I}^{*} f\right)(h) d P=-\int_{\Omega} \operatorname{div}(h) \mathcal{I}^{*}(f) d P=-\int_{\mathcal{C}_{x_{0}} M} \operatorname{div}(V) f d \mu_{x_{0}} \\
= & \int_{\mathcal{C}_{x_{0}} M}(\tilde{d} f)(V) d \mu_{x_{0}}=\int_{\Omega}\left(\mathcal{I}^{*} \tilde{d} f\right)(h) d P
\end{aligned}
$$

which gives $d\left(\mathcal{I}^{*} f\right)=\mathcal{I}^{*}(\tilde{d} f)$.

To see the equivalence of (i) and (iii), take $\frac{1}{r}+\frac{1}{s}=1$. That (i) implies (iii) is trivial. To obtain (i) from (iii), take $f \in W^{r, 1}\left(\mathcal{C}_{x_{0}} M ; \mathbf{R}\right)$, with $\tilde{d} f \in L^{p}$. For any $U \in \mathbb{D}^{s, 1} \mathcal{H}$,

$$
\left|\int \operatorname{div}(U)(\sigma) f(\sigma) \mu(d \sigma)\right|=\left|\int \tilde{d} f(U) \mu(d \sigma)\right| \leq\|\tilde{d} f\|_{L^{p}}|U|_{L^{q}}
$$

which, by continuity using Theorem 5.8 , holds for all $U \in \mathbb{D}^{q, 1} \mathcal{H}$ if $f \in L^{p}$, giving (i).

Corollary 6.2 Suppose Condition $\left(M_{0}\right)$ holds. The symmetric form

$$
\varepsilon(f, g)=\int_{\mathcal{C}_{x_{0}} M}\langle\tilde{d} f, \tilde{d} g\rangle d \mu_{x_{0}}
$$

with domain $W^{2,1}\left(\mathcal{C}_{x_{0}} M ; \mathbf{R}\right)$ is a Dirichlet form.

Proof Just observe that, by Theorem 6.1, the usual chain rule holds for composition on the left by $B C^{1}$ functions on $\mathbf{R}$.

Note that if $\mathcal{I}^{*}\left[\mathbb{D}^{2,1}\left(\mathcal{C}_{x_{0}} M ; \mathbf{R}\right)\right]=\mathbb{D}_{\mathcal{F} x_{0}}^{2,1}(\Omega ; \mathbf{R})$ then, by Theorem $6.1, \mathbb{D}^{2,1}\left(\mathcal{C}_{x_{0}} M ; \mathbf{R}\right)=$ $W^{2,1}\left(\mathcal{C}_{x_{0}} M ; \mathbf{R}\right)$ and $\mathcal{I}^{*} d=d \mathcal{I}^{*}$. In particular we have the Markov uniqueness. Furthermore there is equality of the following two Dirichlet forms:

$$
\int_{\mathcal{C}_{x_{0}} M}|\tilde{d} f|^{2} d \mu_{x_{0}}=\int_{\Omega}\left|\mathbf{E}\left\{d \mathcal{I}^{*}(f) \mid \mathcal{F}^{x_{0}}\right\}\right|^{2} d P
$$

and there is a constant $c$ with

$$
\int_{\mathcal{C}_{x_{0}} M}|\tilde{d} f|^{2} d \mu_{x_{0}} \leq \int_{\Omega}\left|d \mathcal{I}^{*} f\right|^{2} d P \leq c \int_{\mathcal{C}_{x_{0}} M}|\tilde{d} f|^{2} d \mu_{x_{0}}, \quad f \in W^{2,1}\left(\mathcal{C}_{x_{0}} M ; \mathbf{R}\right),
$$

c.f. Driver [11], Shigekawa [41]. 
Corollary 6.3 Suppose Condition $\left(M_{0}\right)$ holds. If $\mathcal{I}^{*}\left[\mathbb{D}^{p, 1}\right]=\mathbb{D}_{\mathcal{F}^{x_{0}}}^{p, 1}$ for the Itô map $\mathcal{I}$ of one stochastic differential equation which induces $\left(\mu_{x_{0}}, \nabla\right)$, then it holds for all such Itô maps.

Proof This is immediate from Theorem 6.1 since $W^{p, 1}$ depend only on $\mu_{x_{0}}$ and $\nabla$.

Proposition 6.4 For $1<p<\infty$,

(1) $\mathbb{D}^{p, 1}\left(\mathcal{C}_{x_{0}} M ; \mathbf{R}\right)$ is a closed subspace of $W^{p, 1}\left(\mathcal{C}_{x_{0}} M ; \mathbf{R}\right)$.

(2) Set $W_{0}^{p, 1}=\left\{f \in W^{p, 1} \mid \int f d \mu_{x_{0}}=0\right\}$. Suppose Condition $\left(M_{0}\right)$ holds. Then $\tilde{d}: W_{0}^{p, 1}\left(\mathcal{C}_{x_{0}} M ; \mathbf{R}\right) \rightarrow L^{p} \Gamma \mathcal{H}^{*}$ has closed range and is a linear isomorphism onto its image.

Proof Part (i) is automatic since $d \subset \tilde{d}$. For (2) the continuity holds by definition of the graph norm while injectivity comes from the result for $\mathcal{C}_{0} \mathbf{R}^{m}$ using Theorem 6.1.

To show $\tilde{d}$ has closed range, take $\phi \in L^{p} \Gamma \mathcal{H}^{*}$ with $\phi=\lim _{j} \tilde{d} f_{j}$ in $L^{p}$, for $f_{j} \in W_{0}^{p, 1}\left(\mathcal{C}_{x_{0}} M ; \mathbf{R}\right)$. Then $\mathcal{I}^{*}\left(\tilde{d} f_{j}\right)=d\left(\mathcal{I}^{*} f_{j}\right)$ by Theorem 6.1 and

$$
\mathcal{I}^{*} \phi=\lim _{j \rightarrow \infty} \mathcal{I}^{*}\left(\tilde{d} f_{j}\right)=\lim _{j \rightarrow \infty} d\left(\mathcal{I}^{*} f_{j}\right)
$$

in $L^{p}$ by Theorem 3.4. Since $d$ on the Wiener space has closed range, e.g. from Shigekawa [42], we see $\mathcal{I}^{*} f_{j} \rightarrow g$ in $\mathbb{D}^{p, 1}(\Omega ; \mathbf{R})$ for some $g \in \mathbb{D}_{\mathcal{F}^{x_{0}}}^{p, \text {. By Theorem }}$ 6.1, $g=\mathcal{I}^{*}(f)$ for some $f \in W_{0}^{p, 1}$ and $f=\lim f_{j}$ in $L^{p}$. Since $\tilde{d}$ is closed, $f \in W_{0}^{p, 1}$ with $\tilde{d} f=\phi$. Thus $\tilde{d}$ has closed range.

\subsection{Markov uniqueness}

Let $\underline{t}=\left\{t_{1}, \ldots, t_{k}\right\}$ with $0 \leq t_{1}<\ldots<t_{k} \leq T$, and write $M \underline{t} \cong M \times \ldots \times M$. A cylindrical $q$-form on $\mathcal{C}_{x_{0}} M$ is of the form $\left(\mathrm{ev}_{\underline{t}}\right)^{*} \varphi$ where $\varphi$ is a smooth $q$-form on $M^{\underline{t}}$ and

$$
\mathrm{ev}_{\underline{\underline{t}}}: \mathcal{C}_{x_{0}} M \rightarrow M^{\underline{t}}
$$

is the evaluation given by

$$
\mathrm{ev}_{\underline{t}}(\sigma)=\left(\sigma\left(t_{1}\right), \ldots, \sigma\left(t_{k}\right)\right) \text {. }
$$

Theorem 6.5 The space $\mathrm{Cyl}^{0} \mathcal{H}^{*}$, defined by (6.3), is total in the space $\mathbb{D}^{q, 1} \mathcal{H}^{*}$ for $1 \leq q<\infty$.

Proof Let $\left(\mathrm{ev}_{\underline{\underline{t}}}\right)^{*} \varphi$ be a typical cylindrical one form on $\mathcal{C}_{x_{0}} M$. In local co-ordinates $\varphi$ can be represented by an expression such as $\sum_{j=1}^{N} \varphi^{j} d x^{j}$, some $N$. It follows using a partition of unity that $\varphi=\Sigma_{j=1}^{N} g^{j} d f^{j}$, for a finite set of smooth cylindrical functions $g^{j}, f^{j}$ on $M^{t}$. Thus $\mathrm{Cyl}^{0} \mathcal{H}^{*}$ spans the space of all smooth cylindrical 1 -forms. The conclusion can be drawn from Proposition 6.14 in $\S 6.3$ below, which states there exists a set of smooth cylindrical 1-forms which is dense in $\mathbb{D}^{q, 1} \mathcal{H}^{*}$. 
Remark 6.6 If our Itô map is induced by a gradient stochastic differential equation then the last part of the proof above is unnecessary since the forms $\varphi^{\tau, e}$ defined in (6.8) would be in $\mathrm{Cyl}^{0} \mathcal{H}^{*}$ as $Y=d j$ for $j$ the immersion of $M$ in $\mathbf{R}^{m}$, and so Proposition 6.14 shows directly that the set

$$
\left\{f \varphi^{\tau, e}: f \text { is } C^{\infty} \text { and cylindrical }, e \in \mathbf{R}^{m}, 0 \leqslant \tau \leq T\right\}
$$

of elements of $\mathrm{Cyl}^{0} \mathcal{H}^{*}$ is total in $\mathbb{D}^{q, 1} \mathcal{H}^{*}$.

Combining Theorem 6.5 with Theorem 5.8 on the continuity of $\operatorname{div}^{q}$ on $\mathbb{D}^{q, 1} \mathcal{H}^{*}$, we see if Condition $\left(M_{0}\right)$ holds,

$$
\overline{\left.\left(d^{p}\right)^{*}\right|_{\mathrm{Cyl}^{0} \mathcal{H}^{*}}}=\overline{\left.\left(d^{p}\right)^{*}\right|_{\mathbb{D}^{q, 1} \mathcal{H}^{*}}}
$$

as operators on $L^{q}$ for $1 \leq q<\infty$. From this,

Theorem 6.7 Suppose Condition $\left(M_{0}\right)$ holds. Let $1<p<\infty$, then

$$
W^{p, 1}={ }^{0} W^{p, 1}
$$

Following Eberle [12], consider the space of bounded functions in ${ }^{0} W^{2,1}$ closed under the weak Sobolev norm, which we shall denote by ${ }^{0} W_{\infty}^{2,1}$.

Proposition 6.8 Suppose Condition $\left(M_{0}\right)$ holds. Then ${ }^{0} W_{\infty}^{2,1}=W^{2,1}$.

Proof Take $f \in{ }^{0} W^{2,1}$ so $f \in W^{2,1}$ by Theorem 6.7. Then $g \equiv f \circ \mathcal{I} \in \mathbb{D}^{2,1}(\Omega)$ by Theorem 6.1. Set $f_{n}=\frac{f}{1+\frac{1}{n} f^{2}}$ and $g_{n}=\mathcal{I}^{*}\left(f_{n}\right)$. Then $\left|f_{n}\right| \leq \frac{1}{2}$ is bounded. Now

$$
d g_{n}(\omega)(h)=\frac{d g(\omega) h}{1+\frac{1}{n} g^{2}} \cdot \frac{n-g^{2}}{n+g^{2}}
$$

is bounded and $g_{n} \in \mathbb{D}^{2,1}(\Omega)$ converges to $g$ in $\mathbb{D}^{2,1}$. Consequently $f_{n} \in{ }^{0} W_{\infty}^{2,1}$ and converges to $f$ in $W^{2,1}$ and so in ${ }^{0} W^{2,1}$. Thus ${ }^{0} W^{2,1}={ }^{0} W_{\infty}^{2,1}$.

Consider the symmetric diffusion operator $L=-d^{*} d$ on $L^{2}\left(\mathcal{C}_{x_{0}} M ; \mathbf{R}\right)$ with domain Cyl, the set of smooth cylindrical functions. It is called Markov unique if and only if there is only one symmetric sub-Markovian $C^{0}$ contraction semi-group $\left(P_{t}\right)$ on $L^{2}\left(\mathcal{C}_{x_{0}} M ; \mathbf{R}\right)$ whose generator extends $L$. Equivalently there is a unique extension of the corresponding Dirichlet form among the family of quasi-regular semi-Dirichlet forms. Markov uniqueness implies that there is at most one reversible diffusion solving the corresponding martingale problem, c.f. Eberle [12], though such results go back to Takeda [46]. We can apply a result of Eberle to our situation to obtain:

Theorem 6.9 Suppose Condition $\left(M_{0}\right)$ holds. The following are equivalent:

1. Markov uniqueness for $-d^{*} d$;

2. $W^{2,1}=\mathbb{D}^{2,1}$;

3. $\mathcal{I}^{*}\left[\mathbb{D}^{2,1}\right]=\mathbb{D}_{\mathcal{F}^{x_{0}}}^{2,1}$.

Proof It follows from Corollary 6.2 and a result of Eberle (page 115, [12]) that Markov uniqueness is equivalent to $\mathbb{D}^{2,1}={ }^{0} W_{\infty}^{2,1}$. Proposition 6.8 then shows that (1) and (2) are equivalent. The equivalence of (2) and (3) is immediate from Theorem 6.1.

For discussion on when $\mathcal{I}^{*}\left[\mathbb{D}^{2,1}\right]=\mathbb{D}_{\mathcal{F}^{x_{0}}}^{2,1}$ holds see Remark 7.4. 


\subsection{Cylindrical 1-forms are dense in $\mathbb{D}^{q, 1} \mathcal{H}^{*}$}

The following lemma will be useful technically, leading to the proof of Theorem 6.5 , in view of the fact that we do not know if $\mathbf{W}^{2,1}=\mathbb{D}^{2,1}$ on our path spaces.

Lemma 6.10 Let $G$ be a separable Hilbert space and $L$ a dense family of linear functionals on $G$. Suppose $f: \mathcal{C}_{x_{0}} M \rightarrow G$ satisfies

(i) $\mathcal{I}^{*}(f) \in \mathbb{D}^{p, 1}\left(\mathcal{C}_{0} \mathbf{R}^{m} ; G\right)$

(ii) $l \circ f \in \mathbb{D}^{p, 1}\left(\mathcal{C}_{x_{0}} M ; \mathbf{R}\right)$ for all $l \in L$.

Then $f \in \mathbb{D}^{p, 1}\left(\mathcal{C}_{x_{0}} M ; G\right)$.

Proof For $f$ satisfying (i) and (ii) and $l \in G^{*}$ take $l_{n} \in L \rightarrow l$. Then

$$
\mathcal{I}^{*}\left(l_{n} f\right)=l_{n}\left(\mathcal{I}^{*} f\right) \stackrel{\mathbb{D}^{p, 1}}{\longrightarrow} l\left(\mathcal{I}^{*} f\right)=\mathcal{I}^{*}(l f) .
$$

By (4.3) $l_{n} f \stackrel{\mathbb{D}^{p, 1}}{\longrightarrow} l f$ and so we have

$$
\text { (iii) } \quad l \circ f \in \mathbb{D}^{p, 1}\left(\mathcal{C}_{x_{0}} M ; \mathbf{R}\right) \text { for all } l \in G^{*} .
$$

Take an orthonormal base $\left\{g_{n}\right\}_{n}$ for $G$ and let $\Pi_{n}$ be the orthogonal projection onto the subspace spanned by its first $n$ elements, $n=1,2, \ldots$ By (iii), $\Pi_{n} \circ f \in$ $\mathbb{D}^{p, 1}\left(\mathcal{C}_{x_{0}} M ; \mathbf{R}\right)$.

Now as $n \rightarrow \infty$ we see $\Pi_{n} \circ f \rightarrow f$ almost surely and also, if $\tilde{d} f$ is as in (6.5), for almost all $\sigma$,

$$
\left\|d\left(\Pi_{n} \circ f\right)_{\sigma}-\tilde{d} f_{\sigma}\right\|_{L_{2}}^{2}=\left\|(\tilde{d} f)_{\sigma}^{*} \Pi_{n}-(\tilde{d} f)_{\sigma}^{*}\right\|_{L_{2}\left(G ; \mathcal{H}_{\sigma}\right)}^{2}=\sum_{p>n}\left\|\tilde{d} f_{\sigma} \circ\left(\tilde{d} f_{\sigma}\right)^{*}\left(g_{p}\right)\right\|^{2}
$$

which converges to zero since $\tilde{d} f_{\sigma} \in G \otimes \mathcal{H}$. From this we can apply the monotone convergence theorem to see that $\Pi_{n} \circ f \rightarrow f$ in the $L^{p}$ graph norm and the result follows.

The next two lemmas are essentially 'well known':

Lemma 6.11 Let $\tilde{\nabla}$ be a connection on the trivial $\mathbf{R}^{k}$-bundle over $M$. Suppose $J$ : $M \rightarrow \mathbb{L}\left(\mathbf{R}^{k} ; \mathbf{R}^{k}\right)$ is $C^{\infty}$ and define the ' $J$-damped' parallel translation $Z_{t}(\sigma):\left\{x_{0}\right\} \times$ $\mathbf{R}^{k} \rightarrow\left\{\sigma_{t}\right\} \times \mathbf{R}^{k}$ along $\sigma$ in $\mathcal{C}_{x_{0}} M$ by

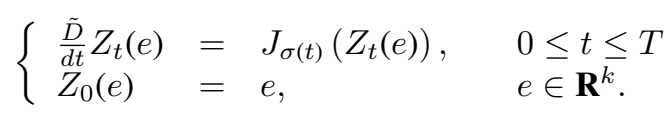

Then the principal part $Z_{t}^{P}$ of $Z_{t}$ as a map from $\mathcal{C}_{x_{0}} M \rightarrow \mathbb{L}\left(\mathbf{R}^{k} ; \mathbf{R}^{k}\right)$ is in $\mathbb{D}^{p, 1}, 1 \leq$ $p<\infty$, for each t. Its $H$-derivative is an $\mathbb{L}\left(\mathbf{R}^{k} ; \mathbf{R}^{k}\right)$-valued $H$-1-form: $h \mapsto d Z_{t}^{P}(\bar{h})$ with

$$
d Z_{t}^{P}(h)=A_{t}\left(W_{t}^{-1} h_{t}\right)+\int_{0}^{t} B_{s}\left(W_{s}^{-1} h_{s}\right) d s+\int_{0}^{t} C_{s}\left(W_{s}^{-1} \frac{\mathbb{D}}{d s} h\right) d s, \quad h \in \mathcal{H}
$$

where

$$
A_{t}, B_{t}, C_{t} \in L^{p}\left(\mathcal{C}_{x_{0}} M ; \mathbb{L}\left(T_{x_{0}} M ; \mathbb{L}\left(\mathbf{R}^{k} ; \mathbf{R}^{k}\right)\right)\right), 1 \leq p<\infty, 0 \leq t \leq T .
$$


Furthermore $A_{t}(\sigma), B_{t}(\sigma)$, and $C_{t}(\sigma): T_{\sigma(t)} M \rightarrow \mathbb{L}\left(\mathbf{R}^{k} ; \mathbf{R}^{k}\right)$ are almost surely continuous in $t$ with

$$
\begin{aligned}
& \mathbf{E}\left(\sup _{0 \leq t \leq T}\left|A_{t}\right|_{\mathbb{L}\left(T_{x_{0}} M ; \mathbb{L}\left(\mathbf{R}^{k} ; \mathbf{R}^{k}\right)\right)}\right)^{p}<\infty, \\
& \mathbf{E}\left(\sup _{0 \leq t \leq T}\left|B_{t}\right|_{\mathbb{L}\left(T_{x_{0}} M ; \mathbb{L}\left(\mathbf{R}^{k} ; \mathbf{R}^{k}\right)\right)}\right)^{p}<\infty, \\
& \mathbf{E}\left(\sup _{0 \leq t \leq T}\left|C_{t}\right|_{\mathbb{L}\left(T_{x_{0}} M ; \mathbb{L}\left(\mathbf{R}^{k} ; \mathbf{R}^{k}\right)\right)}\right)^{p}<\infty .
\end{aligned}
$$

Proof That $Z_{t} \in \mathbb{D}^{p, 1}$ for all $p$ and each $t$ is standard when we are using Brownian motion measure and the Levi-Civita connection, e.g. see the Appendix in Aida [1], or Léandre [33] and the proofs go over to our situation. The computation leading to (6.7) is also standard, going back, at least, to Bismut. See also Driver [9], CruzeiroMalliavin [6]. In particular if $h$ is an adapted $L^{q}-H$-vector field on $\mathcal{C}_{x_{0}} M$, from (6.6), the covariant derivative $\tilde{\nabla}_{h} Z_{t}$ satisfies the covariant Stratonovich equation

$$
\tilde{D} \tilde{\nabla}_{h} Z_{t}(e)=\left[\left(\tilde{\nabla}_{h_{t}} J\right)\left(Z_{t}(e)\right)+J_{\sigma(t)}\left(\tilde{\nabla}_{h} Z_{t}(e)\right)\right] d t-\tilde{R}\left(h_{t}, \circ d \sigma_{t}\right) Z_{t}(e) .
$$

Thus

$$
\tilde{\nabla}_{h} Z_{t}(e)=Z_{t} \int_{0}^{t} Z_{s}^{-1}\left\{\left(\tilde{\nabla}_{h_{s}} J\right)\left(Z_{s}(e)\right) d s-\tilde{R}\left(h_{s}, \circ d \sigma_{s}\right) Z_{s}(e)\right\}
$$

In fact by integration by parts, treating $\int_{0}^{t} Z_{s}^{-1} \tilde{R}\left(W_{s}-, \circ d \sigma_{s}\right) Z_{s}(e)$ as an $L\left(T_{x_{0}} M ; \mathbf{R}^{k}\right)$ valued integral we see

$$
\begin{aligned}
\int_{0}^{t} Z_{s}^{-1} \tilde{R}\left(h_{s}, \circ d \sigma_{s}\right) Z_{s}(e) & =\left(\int_{0}^{t} Z_{s}^{-1} \tilde{R}\left(W_{s}-, \circ d \sigma_{s}\right) Z_{s}(e)\right) W_{t}^{-1} h_{t} \\
- & \int_{0}^{t}\left(\int_{0}^{\tau} Z_{s}^{-1} \tilde{R}\left(W_{s}-, \circ d \sigma_{s}\right) Z_{s}(e)\right)\left(W_{\tau}^{-1} \frac{\mathbb{D}}{d \tau} h_{\tau}\right) d \tau .
\end{aligned}
$$

So our expression for $\tilde{\nabla}_{h} Z_{t}(e)$ is 'tensorial' in $\mathcal{H}$ and so holds for arbitrary elements in $\mathcal{H}$.

To obtain (6.7) we observe that the principal part of $\tilde{\nabla}_{h} Z_{t}(-)$ and $d Z_{t}^{P}(h)$ only differ by $\tilde{\Gamma}(\sigma(t))\left(h_{t}\right)\left(Z_{t}^{p}\right)$ where $\tilde{\Gamma}$ is the Christoffel symbol $\tilde{\Gamma}$. Finally the required estimates come from Proposition 2.2 and the analogous observation that $\left|\sup _{t} Z_{t}\right|$ and $\left|\sup _{t} Z_{t}^{-1}\right|$ are in $L^{p}$ for all $p$.

Lemma 6.12 Let $Z$ be the ' $J$-damped' parallel translation from Lemma 6.11. Then for $p>1$, and $\epsilon \in(0, p-1)$, the map $f \longmapsto Z(f)$ gives a continuous linear map

$$
\mathbb{D}^{p, 1}\left(\mathcal{C}_{x_{0}} M ; L^{2}\left([0, T] ; \mathbf{R}^{k}\right)\right) \rightarrow \mathbb{D}^{p-\varepsilon, 1}\left(\mathcal{C}_{x_{0}} M ; L^{2}\left([0, T] ; \mathbf{R}^{k}\right)\right) .
$$

Proof Take $f$ in $\mathbb{D}^{p, 1}\left(\mathcal{C}_{x_{0}} M ; L^{2}\left([0, T] ; \mathbf{R}^{k}\right)\right)$. It is standard and easy to see using Lemma 6.11 and weak differentiability, e.g. Sugita [45] Corollary 2.1, that $\mathcal{I}^{*}(Z(f)) \in$ $\mathbb{D}^{p-\varepsilon, 1}\left(\mathcal{C}_{0} \mathbf{R}^{m} ; L^{2}\left([0, T] ; \mathbf{R}^{k}\right)\right)$. Let $E_{n}$ be the subspace of $L^{2}\left([0, T] ; \mathbf{R}^{k}\right)$ spanned by the polynomials of degree less than or equal to $n, n=1,2, \ldots$, with $\pi_{n}(Z(f))$ the 
orthogonal projection of $Z(f)$ into $E_{n}$. Since the evaluations $\left\{\operatorname{ev}_{t}, 0 \leq t \leq T\right\}$ span a dense linear subspace of linear functionals on each $E_{n}$, we can apply the previous lemmas to see that $\pi_{n}(Z(f)) \in \mathbb{D}^{p-\varepsilon, 1}\left(\mathcal{C}_{x_{0}} M ; L^{2}\left([0, T] ; \mathbf{R}^{k}\right)\right)$ for each $n=1,2, \ldots$ However as in the proof of Lemma 6.10 we see that $\mathcal{I}^{*}\left(\pi_{n}(Z(f))\right) \rightarrow \mathcal{I}^{*}(Z(f))$ in the $L^{p-\varepsilon}$ graph norm. Therefore $Z(f) \in \mathbb{D}^{p-\varepsilon, 1}\left(\mathcal{C}_{x_{0}} M ; L^{2}\left([0, T] ; \mathbf{R}^{k}\right)\right)$. Continuity follows as usual from continuity into $L^{p-\varepsilon}$ and the closed graph theorem.

For cases including non-elliptic diffusion measures take a Riemannian metric $\langle,\rangle^{\prime}$ on $T M$ extending that of $E$ with a metric connection $\nabla^{1}$ and adjoint $\nabla^{1^{\prime}}$ extending $\nabla$ and $\nabla^{\prime}$ as in $\S 2.2$. Take a surjective vector bundle map $\tilde{X}: \underline{\mathbf{R}}^{\tilde{m}} \rightarrow T M$ for some $\tilde{m}$, inducing the metric $\langle,\rangle^{\prime}$, which extends $X$ if $\mathbf{R}^{m}$ is considered as a subspace of $\mathbf{R}^{\tilde{m}}$. For $x \in M$ let $\tilde{Y}_{x}: T_{x} M \rightarrow \mathbf{R}^{\tilde{m}}$ be the usual right inverse of $\tilde{X}(x)$. Take a connection $\tilde{\nabla}$ on $\mathbf{R}^{\tilde{m}}$ conjugate to $\nabla^{1^{\prime}}$ on $[\operatorname{ker} \tilde{X}]^{\perp}$ and arbitrary on ker $\tilde{X}$. In the elliptic case we could take $\tilde{X}=X, \tilde{Y}=Y$ and if moreover $\nabla$ is the Levi-Civita connection then $\tilde{\nabla}$ could be the standard metric connection on $\mathbf{R}^{m}$ induced by $K$, as in Elworthy-LeJan-Li [15].

In order to prove the density of cylindrical forms in $\mathbb{D}^{q, 1} \mathcal{H}^{*}$ fix $\tau \in[0, T]$ and $e \in \mathbf{R}^{\tilde{m}}$. Let $\varphi^{\tau, e}$ be the cylindrical one-form given by

$$
\varphi^{\tau, e}(V)=\left\langle\tilde{Y}_{\sigma(\tau)}\left(V_{\tau}\right), e\right\rangle, \quad V \in T_{\sigma} \mathcal{C}_{x_{0}} M
$$

In fact we will show that the set $\left\{f \phi^{\tau, e}: f \in \mathrm{Cyl}, e \in \mathbf{R}^{\tilde{m}}, 0 \leq \tau \leq T\right\}$ is total in $\mathbb{D}^{q, 1} \mathcal{H}^{*}$. Set

$$
U^{\tau, e}=\left(\varphi^{\tau, e}\right)^{\#} .
$$

Using the fact that

$$
W_{\tau} \int_{0}^{\tau} W_{s}^{-1} \frac{\mathbb{D}}{d s} V_{s} d s=V \tau
$$

we see that

$$
\frac{\mathbb{D}}{d s} U_{s}^{\tau, e}=\chi_{[0, \tau]}(s) \Pi_{s}\left(W_{s}^{-1}\right)^{*}\left(W_{\tau}\right)^{*} \tilde{X}(\sigma(\tau))(e),
$$

where $\Pi_{s}=\Pi_{s}(\sigma): T_{\sigma(s)} M \rightarrow E_{\sigma(s)}$ is the orthogonal projection and $W_{s}^{*}$ and $W_{s}^{-1^{*}}$ the adjoints using the extended metric $\langle,\rangle^{\prime}$.

Now define $Z_{t} \equiv Z_{t}(\sigma): \mathbf{R}^{\tilde{m}} \rightarrow \mathbf{R}^{\tilde{m}}$ to be the damped parallel translation on the trivial $\mathbf{R}^{\tilde{m}}$-bundle over $M$ along $\sigma \in \mathcal{C}_{x_{0}} M$ given by

$$
Z_{t}(a)= \begin{cases}\tilde{Y}_{\sigma(t)} W_{t} \tilde{X}\left(x_{0}\right) a, & \text { if } a \in\left[\operatorname{ker}\left(\tilde{X}\left(x_{0}\right)\right)\right]^{\perp} \\ \tilde{I}_{t}(a), & \text { if } a \in \operatorname{ker}\left(\tilde{X}\left(x_{0}\right)\right) .\end{cases}
$$

By (2.10), $Z_{t}$ solves (6.6) for

$$
J(x)(a)=\tilde{Y}(x)\left(-\frac{1}{2} R i c_{x}^{\#}+\nabla_{-}^{1} A\right) \tilde{X}(x) a .
$$

Let $Z_{t}^{*}: \mathbf{R}^{\tilde{m}} \rightarrow \mathbf{R}^{\tilde{m}}$ be the usual adjoint of $Z_{t}$ and similarly for $\left(Z_{t}^{-1}\right)^{*}$. Set

$$
\Lambda_{s}^{\tau, e}=\chi_{[0, \tau]}(s)\left(Z_{s}^{-1}\right)^{*} Z_{\tau}^{*} e
$$

then

$$
\frac{\mathbb{D}}{d s} U_{s}^{\tau, e}=\Pi_{s} \tilde{X}(\sigma(s)) \Lambda_{s}^{\tau, e}
$$


Lemma 6.13 Set

$$
\Xi=\left\{f \Lambda^{\tau, e}: f \in \mathrm{Cyl}, e \in \mathbf{R}^{\tilde{m}}, 0 \leq \tau \leq T\right\} .
$$

The sets $Z^{*}[\Xi]$ and $\Xi$ are both total in $\mathbb{D}^{q, 1}\left(\mathcal{C}_{x_{0}} M ; L^{2}\left([0, T] ; \mathbf{R}^{\tilde{m}}\right)\right)$.

Proof Since every element of the Haar basis, $\left\{E^{j}\right\}_{j}$ say, of $L^{2}\left([0, T] ; \mathbf{R}^{\tilde{m}}\right)$ has the form $\chi_{\left[0, \tau_{2}\right]}(\cdot) e-\chi_{\left[0, \tau_{1}\right]}(\cdot) e$ some $0 \leq \tau_{1} \leq \tau_{2} \leq T, e \in \mathbf{R}^{\tilde{m}}$, using the definition of $\mathbb{D}^{q, 1}$ we have

$$
\mathcal{T}:=\left\{f \chi_{[0, \tau]}(\cdot) e: 0 \leq \tau \leq T, e \in \mathbf{R}^{\tilde{m}}, f \in \mathrm{Cyl}\right\}
$$

is total in $\mathbb{D}^{q, 1}\left(\mathcal{C}_{x_{0}} M ; L^{2}\left([0, T] ; \mathbf{R}^{\tilde{m}}\right)\right)$ for $1 \leq q<\infty$. On the other hand, by definition,

$$
Z^{*}[\Xi]=\left\{f \chi_{[0, \tau]}(\cdot) Z_{\tau}^{*} e .: f \in \mathrm{Cyl}, e \in \mathbf{R}^{\tilde{m}}, 0 \leq \tau \leq T\right\},
$$

a subset of $\mathbb{D}^{q, 1}\left(\mathcal{C}_{x_{0}} M ; L^{2}\left([0, T] ; \mathbf{R}^{\tilde{m}}\right)\right.$ from Lemma 6.10 .

For each $e \in \mathbf{R}^{\tilde{m}}$ and $\tau \in[0, T]$ define $e^{\tau}: \mathcal{C}_{x_{0}} M \rightarrow \mathbf{R}^{\tilde{m}}$ by

$$
e^{\tau}(\sigma)=\left(Z_{\tau}^{*}(\sigma)\right)^{-1} e .
$$

Note that $e^{\tau} \in \mathbb{D}^{p, 1}, 1 \leq p<\infty$, by Lemma 6.10 (applied to $\left.\left(Z^{-1}\right)^{*}\right)$. Consequently given $\varepsilon>0$ and $q_{0}>1$, there exist $g^{\tau} \in \operatorname{Cyl}\left(C_{x_{0}} M ; \mathbf{R}^{\tilde{m}}\right)$ with

$$
\left\|g^{\tau}-e^{\tau}\right\|_{\mathbb{D}^{q, 1}}<\varepsilon, \quad 1 \leq q \leq q_{0} .
$$

Then, for $1 \leq q<q_{0}$,

$$
\begin{aligned}
& \left\|\chi_{[0, \tau]}(\cdot) e-\chi_{[0, \tau]}(\cdot) Z_{\tau}^{*} g^{\tau}\right\|_{\mathbf{D}^{q, 1}\left(\mathcal{C}_{x_{0}} M ; L^{2}\left([0, T] ; \mathbf{R}^{\tilde{m}}\right)\right)} \\
= & \left\|\chi_{[0, \tau]}(\cdot)\left(Z_{\tau}^{*} e^{\tau}-Z_{\tau}^{*} g^{\tau}\right)\right\|_{\mathbb{D}^{q, 1}\left(\mathcal{C}_{x_{0}} M ; L^{2}\left([0, T] ; \mathbf{R}^{\tilde{m}}\right)\right)} \leq C \varepsilon\left\|Z_{\tau}\right\|_{\mathbb{D}^{r, 1}}
\end{aligned}
$$

for sufficiently large $r$ and some constant $C$.

Thus each $\chi_{[0, \tau]}(\cdot) e \in \overline{\operatorname{Span}}^{q, 1} Z^{*}[\Xi], 1 \leq q<\infty$ and $\mathcal{T} \subset \overline{\operatorname{Span}}^{q, 1} Z^{*}[\Xi]$. Consequently for each $1 \leq q<\infty$,

$$
\overline{\operatorname{Span}}^{q, 1} Z^{*}[\Xi]=\mathbb{D}^{q, 1}\left(\mathcal{C}_{x_{0}} M ; L^{2}\left([0, T] ; \mathbf{R}^{\tilde{m}}\right)\right),
$$

as required for the first assertion. For the second, take $f E^{j} \in \operatorname{Span} \mathcal{T}$ and $q<p<\infty$. By Lemma $6.12 Z^{*}\left(f E^{j}\right) \in \mathbb{D}^{p, 1}$. Since by the first assertion $\mathbb{D}^{p, 1}=\operatorname{Span} Z^{*}[\Xi]$, there is a sequence $\left\{S^{n}\right\}_{n}$ in $\operatorname{Span}[\Xi]$ with $Z_{*}^{*}\left(S_{*}^{n}\right) \rightarrow Z^{*}\left(f E^{j}\right)$ in $\mathbb{D}^{p, 1}$. Using Lemma 6.12, applied to $\left(Z^{*}\right)^{-1}$, we see $S^{n} \rightarrow f E^{j}$ in $\mathbb{D}^{q, 1}$, which implies the result by the totality of $\mathcal{T}$.

Proposition 6.14 Smooth cylindrical 1-forms form a dense subspace of $\mathbb{D}^{q, 1} \mathcal{H}^{*}$.

Proof By construction of $\tilde{X}$, if $\pi: \mathbf{R}^{\tilde{m}} \rightarrow \mathbf{R}^{\tilde{m}}$ is the projection map then $\Pi \tilde{X}=X \circ \pi$ and so

$$
\mathbb{D}^{q, 1} \mathcal{H}=\left\{\mathbf{W}(\tilde{X}(h)) \mid h \in \mathbb{D}^{q, 1}\left(\mathcal{C}_{x_{0}} M ; L^{2}\left([0, T] ; \mathbf{R}^{\tilde{m}}\right)\right\} .\right.
$$

By Lemma 6.13, $\left\{\mathbf{W} . \Pi \tilde{X}\left(f \Lambda^{\tau, e}\right): f \in \mathrm{Cyl}, e \in \mathbf{R}^{\tilde{m}}, 0 \leq \tau \leq T\right\}$ is total in $\mathbb{D}^{q, 1} \mathcal{H}$. Finally note that by $(6.11), \mathbf{W} . \Pi \tilde{X}\left(f \Lambda^{\tau, e}\right)=U_{s}^{\tau, e}$ and so the set

$$
\left\{f \phi^{\tau, e}: f \in \mathrm{Cyl}, e \in \mathbf{R}^{\tilde{m}}, 0 \leq \tau \leq T\right\}
$$


is total in $\mathbb{D}^{q, 1} \mathcal{H}^{*}$.

To see that every cylindrical one-form gives an element of $\cap_{q<\infty} \mathbb{D}^{q, 1} \mathcal{H}$ suppose $\phi$ is one, given by

$$
\phi_{\sigma}(v)=\Phi_{\left(\sigma\left(t_{1}\right), \ldots, \sigma\left(t_{k}\right)\right)}\left(v_{t_{1}}, \ldots, v_{t_{k}}\right), \quad v \in \mathcal{H}_{\sigma}
$$

where $\Phi$ is a smooth 1-form on $\overbrace{M \times \ldots \times M}^{k}$. We must show

$$
\phi \circ \overline{T \mathcal{I}} \in \mathbb{D}^{q, 1}\left(\mathcal{C}_{x_{0}} M ; H^{*}\right), \quad 1<q<\infty .
$$

For this write

$$
\begin{aligned}
\phi \circ T \mathcal{I}_{\sigma}(h)= & \Phi\left(\tilde{X}\left(\sigma\left(t_{1}\right)\right)-, \ldots, \tilde{X}\left(\sigma\left(t_{k}\right)\right)-\right) \\
& \left(\int_{0}^{t_{1}} \tilde{Y}_{\sigma\left(t_{1}\right)} W_{t_{1}}^{s} \tilde{X}(\sigma(s)) \dot{h}_{s} d s, \ldots, \int_{0}^{t_{k}} \tilde{Y}_{\sigma\left(t_{k}\right)} W_{t_{k}}^{s} \tilde{X}(\sigma(s)) \dot{h}_{s} d s\right)
\end{aligned}
$$

for $h \in H \subset L_{0}^{2,1}\left([0, T] ; \mathbf{R}^{\tilde{m}}\right)$. The first part of the right hand side is in

$$
\cap_{q<\infty} \mathbb{D}^{q, 1}\left(\mathcal{C}_{x_{0}} M,\left(\mathbf{R}^{\tilde{m}} \times \ldots \times \mathbf{R}^{\tilde{m}}\right)^{*}\right),
$$

being cylindrical. The second can be written as

$$
h \mapsto\left(\int_{0}^{t_{1}} Z_{t_{1}} Z_{s}^{-1} K^{\perp}(\sigma(s)) \dot{h}_{s} d s, \ldots, \int_{0}^{t_{k}} Z_{t_{k}} Z_{s}^{-1} K^{\perp}(\sigma(s)) \dot{h}_{s} d s\right)
$$

which by Lemma 6.12 and 5.6 gives a continuous linear map

$$
H \rightarrow \mathbb{D}^{q, 1}\left(\mathcal{C}_{x_{0}} M,\left(\mathbf{R}^{\tilde{m}} \times \ldots \times \mathbf{R}^{\tilde{m}}\right) .\right.
$$

Thus $\phi \in \mathbb{D}^{q, 1}$.

\section{On uniqueness of $d$}

\subsection{A weak uniqueness result on the Gross derivative operator $d$}

If Condition $\left(M_{0}\right)$ holds the map $\mathcal{I}^{*}$ sends $\mathbb{D}^{2,1}\left(\mathcal{C}_{x_{0}} M ; \mathbf{R}\right)$ to $\mathbb{D}_{\mathcal{F} x_{0}}^{2,1}(\Omega ; \mathbf{R})$ with closed range, by Corollary 4.3. We investigate the question whether $\mathcal{I}^{*}\left[\mathbb{D}^{2,1}\left(\mathcal{C}_{x_{0}} M ; \mathbf{R}\right)\right]=$ $\mathbb{D}_{\mathcal{F}_{0}}^{2,1}(\Omega ; \mathbf{R})$. We proceed using chaos expansions. An $L^{2}$ real valued function $f$ on the Wiener space has a chaos expansion

$$
f=\sum_{k=0}^{\infty} I_{k}\left(\alpha_{k}\right)
$$

for $\mathcal{I}_{0}=$ Id on constants, $\alpha_{0}=\mathbf{E}(f), I_{1}\left(\alpha_{1}\right)=\int_{0}^{T}\left\langle\alpha_{1}\left(t_{1}\right), d B_{t_{1}}\right\rangle$, and for $k>1$,

$$
I_{k}\left(\alpha_{k}\right)=k ! \int_{0}^{T} \int_{0}^{t_{k}} \ldots \int_{0}^{t_{2}}\left\langle\alpha_{k}\left(t_{1}, \ldots, t_{k}\right), d B_{t_{1}} \otimes \ldots \otimes d B_{t_{k}}\right\rangle_{\otimes \mathbf{R}^{m}},
$$

an iterated Itô integral. Here $\alpha_{k}$ is considered to be an element of $L^{2}\left(\Delta^{k} ; \mathbf{R}^{m} \otimes \ldots \otimes \mathbf{R}^{m}\right)$ for $\Delta^{k}=\left\{\left(t_{1}, \ldots, t_{k}\right), 0 \leq t_{1} \leq t_{2} \leq \ldots t_{k} \leq T\right\}$. 
Let $R_{n}$ be the remainder term such that

$$
f=\sum_{k=0}^{n} I_{k}\left(\alpha_{k}\right)+R_{n}
$$

Then $f \in \mathbb{D}^{2,1}(\Omega ; \mathbf{R})$ if and only if $R_{n} \rightarrow 0$ in $L^{2}$ and $\left\|d R_{n}\right\| \rightarrow 0$ in $L^{2}$ (e.g. see Nualart [38], Proposition 1.2.2).

Let $L_{\mathcal{F}^{x} 0}^{2} \equiv L_{\mathcal{F}^{x_{0}}}^{2}(\Omega, \mathbf{R})$ be the closed subspace of $L^{2}(\Omega ; \mathbf{R})$ whose elements are $\mathcal{F}^{x_{0}}$ measurable.

Lemma 7.1 If $f \in \mathbb{D}_{\mathcal{F}^{x_{0}}}^{2,1}(\Omega, \mathbf{R})$ then the remainder term of its $L^{2}$ chaos expansion has the following form

$$
R_{k}=\int_{0}^{T} \int_{0}^{s_{k+1}} \ldots \int_{0}^{s_{2}}\left\langle a_{k+1}\left(s_{1}, \ldots, s_{k+1}\right), d B_{s_{1}} \otimes \ldots \otimes d B_{s_{k+1}}\right\rangle
$$

for $a_{k+1} \in L^{2}\left(\mathcal{C}_{0} \mathbf{R}^{m} \times \Delta^{k+1} ; \mathbf{R}^{m} \otimes \ldots \otimes \mathbf{R}^{m}\right)$ such that

(i) Each $a_{k+1}\left(s_{1}, \ldots, s_{k+1}\right)$ is $\mathcal{F}_{s_{1}}^{x_{0}}$ measurable;

(ii) $a_{k+1}\left(s_{1}, \ldots, s_{k+1}\right)=\left(K^{\perp}\left(x_{s_{1}}\right) \otimes \mathbf{1} \ldots \otimes \mathbf{1}\right) \alpha^{k+1}\left(s_{1}, \ldots s_{k+1}\right)$, where $K^{\perp}(x)$ : $\mathbf{R}^{m} \rightarrow[\operatorname{ker} X(x)]^{\perp}$ is the orthogonal projection.

(iii) Furthermore $a_{k+1} \in \mathbb{D}^{2,1}\left(\mathcal{C}_{0} \mathbf{R}^{m} ; L^{2}\left(\Delta^{k+1} ; \mathbf{R}^{m} \otimes \ldots \otimes \mathbf{R}^{m}\right)\right)$ and

$$
\left\|d R_{k}\right\|_{L^{2}}^{2}=(k+1)\left\|a_{k+1}\right\|_{L^{2}}^{2}+\left\|d a_{k+1}\right\|_{L^{2}}^{2} .
$$

Proof By the integral representation theorem there exists an $L^{2}, \mathcal{F}_{*}$-adapted process $a_{1}:[0, T] \times \mathcal{C}_{0} \mathbf{R}^{m} \rightarrow \mathbf{R}^{m}$ with

$$
f=\mathbf{E} f+\int_{0}^{T}\left\langle a_{1}(s), d B_{s}\right\rangle
$$

Since $f=\mathbf{E}\left\{f \mid \mathcal{F}^{x_{0}}\right\}$ we see, using (3.2),

$$
f=\mathbf{E} f+\int_{0}^{T}\left\langle K^{\perp}\left(x_{s}\right) \mathbf{E}\left\{a_{1}(s) \mid \mathcal{F}^{x_{0}}\right\}, d B_{s}\right\rangle .
$$

By the uniqueness of the integral representation (7.4)

$$
K^{\perp}\left(x_{s}\right) \mathbf{E}\left\{a_{1}(s) \mid \mathcal{F}^{x_{0}}\right\}=a_{1}(s) .
$$

Thus $a_{1}(s)$ is $\mathcal{F}_{s}^{x_{0}}$ measurable and (i) and (ii) hold for $k=0$. Suppose for induction (i) and (ii) hold for $k-1$ some $k \geq 1$. Apply the integral representation theorem to the $\mathcal{F}_{s_{2}}^{x_{0}}$ measurable function $a_{k}\left(s_{2}, \ldots, s_{k+1}\right)$ to see

$$
a_{k}\left(s_{2}, \ldots, s_{k}\right)=\mathbf{E}\left(a_{k}\left(s_{2}, \ldots, s_{k}\right)\right)+\int_{0}^{s_{2}}\left\langle a_{k+1}\left(s_{1}, \ldots, s_{k+1}\right), d B_{s_{1}}\right\rangle,
$$

where $a_{k+1}\left(s_{1}, \ldots, s_{k+1}\right)$ is $\mathcal{F}_{s_{1}}^{x_{0}}$ measurable with

$$
\left(K^{\perp}\left(x_{s_{1}}\right) \otimes \mathbf{1} \ldots \otimes \mathbf{1}\right) a_{k+1}\left(s_{1}, \ldots, s_{k+1}\right)=a_{k+1}\left(s_{1}, \ldots, s_{k+1}\right) .
$$


By the uniqueness of the chaos expansion with remainder terms, we see that $R_{k}$ together with $a_{k+1}$ satisfies (i) and (ii).

To prove part (iii), we apply a result of Pardoux-Peng [39], see Lemma 1.3.4 in Nualart [38]: if

$$
X=\int_{0}^{T}\left\langle u_{s}, d B_{s}\right\rangle
$$

where $u$ is adapted and square integrable then $X \in \mathbb{D}^{2,1}$ implies $u . \in \mathbb{D}^{2,1}$. Moreover

$$
\|d X\|_{L^{2}}^{2}=\int_{0}^{T} \mathbf{E}\left|u_{s}\right|^{2} d s+\int_{0}^{T}\left\|d u_{s}\right\|_{L^{2}}^{2} d s .
$$

Apply this to $R_{k}$ iteratively, to deduce that $a_{k+1}\left(s_{1}, \ldots, s_{k+1}\right)$ belongs to $\mathbb{D}^{2,1}$ and

$$
\begin{aligned}
& \left\|d R_{k}\right\|_{L^{2}}^{2} \\
& =\int_{0}^{T} \mathbf{E}\left|\int_{0}^{s_{k+1}} \ldots \int_{0}^{s_{2}}\left\langle a_{k+1}\left(s_{1}, \ldots, s_{k+1}\right), d B_{s_{1}} \otimes \ldots \otimes d B_{s_{k}}\right\rangle\right|^{2} d s_{k+1} \\
& +\int_{0}^{T}\left\|d \int_{0}^{s_{k+1}} \int_{0}^{s_{k}} \ldots \int_{0}^{s_{2}}\left\langle a_{k+1}\left(s_{1}, \ldots, s_{k+1}\right), d B_{s_{1}} \otimes \ldots \otimes d B_{s_{k}}\right\rangle\right\|_{L^{2}}^{2} d s_{k+1} \\
& =\left\|a_{k+1}\right\|_{L^{2}}^{2}+\int_{0}^{T} \int_{0}^{s_{k+1}} \mathbf{E}\left|\int_{0}^{s_{k}} \ldots \int_{0}^{s_{2}}\left\langle a_{k+1}\left(s_{1}, \ldots, s_{k+1}\right), d B_{s_{1}} \otimes \ldots \otimes d B_{s_{k-1}}\right\rangle\right|^{2} d s_{k} d s_{k+1} \\
& +\int_{0}^{T} \int_{0}^{s_{k+1}}\left\|d \int_{0}^{s_{k}} \int_{0}^{s_{k-1}} \ldots \int_{0}^{s_{2}}\left\langle a_{k+1}\left(s_{1}, \ldots, s_{k+1}\right), d B_{s_{1}} \otimes \ldots \otimes d B_{s_{k-1}}\right\rangle\right\|_{L^{2}}^{2} d s_{k} d s_{k+1} \\
& =\ldots
\end{aligned}
$$

giving (7.3).

Note that Lemma 7.1 (iii) holds if $\mathcal{F}^{x_{0}}$ is replace by $\mathcal{F}_{T}$ everywhere in the statement. This shows that a function $f \in \mathbb{D}^{2,1}$ if and only if

$$
k\left\|a_{k}\right\|_{L^{2}}^{2} \rightarrow 0 \text { and }\left\|d a_{k}\right\|_{L^{2}}^{2} \rightarrow 0 .
$$

Lemma 7.2 Let $f \in L^{2}(\Omega ; \mathbf{R})$ with chaos expansion $f=\sum_{k=0}^{\infty} I_{k}\left(\alpha_{k}\right)$. Set $\bar{J}_{k}\left(\alpha_{k}\right)(\sigma)=$ $\mathbf{E}\left\{\mathcal{I}_{k}\left(\alpha_{k}\right) \mid x .=\sigma\right\}$. Then $\bar{J}_{k}\left(\alpha_{k}\right)$ is in $\mathbb{D}^{2,1}\left(\mathcal{C}_{x_{0}} M\right)$. Consequently if $f \in \mathbb{D}_{\mathcal{F}^{x_{0}}}^{2,1}$ then each $f-\mathbf{E}\left\{R_{k} \mid \mathcal{F}^{x_{0}}\right\}$ is in $\mathcal{I}^{*}\left[\mathbb{D}^{2,1}\left(\mathcal{C}_{x_{0}} M ; \mathbf{R}\right)\right]$.

Proof For $0 \leq t \leq T$ define

$$
I_{k, t}\left(\alpha_{k}\right):=k ! \int_{0}^{t} \int_{0}^{t_{k}} \ldots \int_{0}^{t_{2}}\left\langle\alpha_{k}\left(t_{1}, \ldots, t_{k}\right), d B_{t_{1}} \otimes \ldots \otimes d B_{t_{k}}\right\rangle_{\otimes \mathbf{R}^{m}} .
$$

Thus

$$
I_{k, t}\left(\alpha_{k}\right)=k \int_{0}^{t}\left\langle I_{k-1, t_{k}}\left(\alpha_{k}\left(\ldots, t_{k}\right)\right), d B_{t_{k}}\right\rangle_{\mathbf{R}^{m}}
$$

considering $s \mapsto I_{k-1, t_{k}}\left(\alpha_{k}(\ldots, s)\right)$ as a random element in $L^{2}\left([0, T] ; \mathbf{R}^{m}\right)$, or more precisely

$$
I_{k, t}\left(\alpha_{k}\right)=k \int_{0}^{t}\left\langle\left(I_{k-1, t_{k}} \otimes i d\right)\left(\alpha_{k}\left(\ldots, t_{k}\right)\right), d B_{t_{k}}\right\rangle_{\mathbf{R}^{m}}
$$


if we identified $L^{2}(\Omega ; \mathbf{R}) \otimes \mathbf{R}^{m}$ with $L^{2}\left(\Omega ; \mathbf{R}^{m}\right)$. Set

$$
\begin{aligned}
J_{k, t}\left(\alpha_{k}\right) & =\mathbf{E}\left\{I_{k, t}\left(\alpha_{k}\right) \mid \mathcal{F}^{x_{0}}\right\}, k=0,1,2, \ldots \\
J_{k}\left(\alpha_{k}\right) & =J_{k, T}\left(\alpha_{k}\right) .
\end{aligned}
$$

Then inductively

$$
J_{k, t}\left(\alpha_{k}\right)=k \int_{0}^{t}\left\langle J_{k-1, t_{k}}\left(\alpha_{k}\left(\ldots, t_{k}\right)\right), K^{\perp}\left(x_{t_{k}}\right) d B_{t_{k}}\right\rangle_{\mathbf{R}^{m}}
$$

Set $\breve{B}_{t}=\int_{0}^{t} / / s^{-1} X\left(x_{s}\right) d B_{s}$, the martingale part of the anti-development of $x$. to see

$$
\begin{aligned}
K^{\perp}\left(x_{t}\right) d B_{t} & =Y\left(x_{t}\right) X\left(x_{t}\right) d B_{t}=Y\left(x_{t}\right) / / t(x .) d \breve{B}_{t} \\
\bar{J}_{k, t}\left(\alpha_{k}\right)(\sigma) & =k \int_{0}^{t}\left\langle\bar{J}_{k-1, t_{k}}\left(\alpha_{k}\left(\ldots, t_{k}\right)\right)(\sigma), Y\left(x_{t_{k}}\right) / / t_{k}(\sigma .) d \breve{B}_{t_{k}}\right\rangle_{\mathbf{R}^{m}}
\end{aligned}
$$

The fact that $\bar{J}_{k, t}\left(\alpha_{k}\right)$ is in $\operatorname{Dom}(d)$ is essentially standard $e . g$. see Cruzeiro-Malliavin [6] or the Appendix in Aida [1]. For a gradient stochastic differential equation (2.5) determined by an isometric $j: M \rightarrow \mathbf{R}^{m}$ it is especially clear since then $K^{\perp}\left(x_{t}\right) d B_{t}$ can be replaced by $d \tilde{x}_{t}-\frac{1}{2} \Delta j\left(x_{t}\right) d t$ for $\tilde{x}_{t}=j\left(x_{t}\right) \in \mathbf{R}^{m}$.

Finally just observe that for $f \in \mathbb{D}_{\mathcal{F}^{x_{0}}}^{2,1}$,

$$
f-\mathbf{E}\left\{R_{k} \mid \mathcal{F}^{x_{0}}\right\}=\sum_{j=1}^{m} \mathcal{I}^{*}\left(\bar{J}_{k}\left(\alpha_{k}\right)\right)
$$

which is in $\mathcal{I}^{*}\left[\mathbb{D}^{2,1}\left(\mathcal{C}_{x_{0}} M\right)\right]$.

Proposition 7.3 Suppose Condition $\left(M_{0}\right)$ holds. Then $f \in \operatorname{Dom}(\Delta) \cap L_{\mathcal{F}^{x_{0}}}^{2}(\Omega, \mathbf{R})$ implies that $f \in \mathcal{I}^{*}\left[\mathbb{D}^{2,1}\left(\mathcal{C}_{x_{0}} M ; \mathbf{R}\right)\right]$.

Proof Take $f \in \mathbb{D}^{2,1}(\Omega, \mathbf{R})$. By Corollary $4.3, \mathcal{I}^{*}\left[\mathbb{D}^{2,1}\left(\mathcal{C}_{x_{0}} M\right)\right]$ is closed in $\mathbb{D}^{2,1}\left(\mathcal{C}_{0} \mathbf{R}^{m} ; \mathbf{R}\right)$ and so by Lemma 7.2 to show $f \in \mathcal{I}^{*}\left[\mathbb{D}^{2,1}\left(\mathcal{C}_{x_{0}} M ; \mathbf{R}\right)\right.$ we only need to demonstrate that $\left\|d\left(\mathbf{E}\left\{R_{k} \mid \mathcal{F}^{x_{0}}\right\}\right)\right\|_{L^{2}} \rightarrow 0$. Observe that, as iterated integrals,

$$
\begin{aligned}
\mathbf{E}\left\{R_{k} \mid \mathcal{F}^{x_{0}}\right\}= & \int_{0}^{T} \int_{0}^{s_{k+1}} \ldots \int_{0}^{s_{2}} \\
& \left\langle a_{k+1}\left(s_{1}, \ldots, s_{k+1}\right), K^{\perp}\left(x_{s_{1}}\right) d B_{s_{1}} \otimes \ldots \otimes K^{\perp}\left(x_{s_{k+1}}\right) d B_{s_{k+1}}\right\rangle
\end{aligned}
$$

and for $h \in H$,

$$
\begin{aligned}
& d\left(\mathbf{E}\left\{R_{k} \mid \mathcal{F}^{x_{0}}\right\}\right)(h) \\
& =\sum_{l=1}^{k+1} \int_{0}^{T} \int_{0}^{s_{k+1}} \ldots \int_{0}^{s_{2}}\left\langle a_{k+1}\left(s_{1}, \ldots, s_{k+1}\right),\right. \\
& \left.\quad K^{\perp}\left(x_{s_{1}}\right) d B_{s_{1}} \otimes \ldots \otimes d\left(K^{\perp}\left(\mathcal{I}_{s_{l}}\right)\right)(h) d B_{s_{l}} \otimes \ldots K^{\perp}\left(x_{s_{k+1}}\right) d B_{s_{k+1}}\right\rangle \\
& +\sum_{l=1}^{k+1} \int_{0}^{T} \int_{0}^{s_{k+1}} \ldots \int_{0}^{s_{2}}\left\langle a_{k+1}\left(s_{1}, \ldots, s_{k+1}\right),\right.
\end{aligned}
$$




$$
\begin{gathered}
\left.K^{\perp}\left(x_{s_{1}}\right) d B_{s_{1}} \otimes \ldots K^{\perp}\left(x_{s_{l}}\right) \dot{h}_{s_{l}} \otimes \ldots K^{\perp}\left(x_{s_{k+1}}\right) d B_{s_{k+1}}\right\rangle \\
+\int_{0}^{T} \int_{0}^{s_{k+1}} \ldots \int_{0}^{s_{2}}\left\langle d\left(a_{k+1}\left(s_{1}, \ldots, s_{k+1}\right)\right)(h), K^{\perp}\left(x_{s_{1}}\right) d B_{s_{1}} \otimes \ldots K^{\perp}\left(x_{s_{k+1}}\right) d B_{s_{k+1}}\right\rangle \\
=A_{1}(h)+A_{2}(h) .
\end{gathered}
$$

where

$$
\begin{aligned}
A_{1}(h)= & \sum_{l=1}^{k+1} \int_{0}^{T} \int_{0}^{s_{k+1}} \ldots \int_{0}^{s_{2}}\left\langle a_{k+1}\left(s_{1}, \ldots, s_{k+1}\right),\right. \\
& \left.K^{\perp}\left(x_{s_{1}}\right) d B_{s_{1}} \otimes \ldots \otimes d\left(K^{\perp} \circ \mathcal{I}_{s_{l}}\right)(h) d B_{s_{l}} \otimes \ldots K^{\perp}\left(x_{s_{k+1}}\right) d B_{s_{k+1}}\right\rangle
\end{aligned}
$$

On the other hand

$$
\begin{gathered}
\mathbf{E}\left\{d R_{k}(h) \mid \mathcal{F}^{x_{0}}\right\}=\sum_{l=1}^{k+1} \int_{0}^{T} \int_{0}^{s_{k+1}} \ldots \int_{0}^{s_{2}}\left\langle a_{k+1}\left(s_{1}, \ldots, s_{k+1}\right),\right. \\
\left.K^{\perp}\left(x_{s_{1}}\right) d B_{s_{1}} \otimes \ldots \dot{h}_{s_{l}} \otimes \ldots K^{\perp}\left(x_{s_{k+1}}\right) d B_{s_{k+1}}\right\rangle \\
+\int_{0}^{T} \int_{0}^{s_{k+1}} \ldots \int_{0}^{s_{2}}\left\langle\mathbf{E}\left\{d\left(a_{k+1}\left(s_{1}, \ldots, s_{k+1}\right)\right)(h) \mid \mathcal{F}^{x_{0}}\right\},\right. \\
\left.K^{\perp}\left(x_{s_{1}}\right) d B_{s_{1}} \otimes \ldots K^{\perp}\left(x_{s_{k+1}}\right) d B_{s_{k+1}}\right\rangle .
\end{gathered}
$$

Thus

$$
d\left(\mathbf{E}\left\{R_{k} \mid \mathcal{F}^{x_{0}}\right\}\right)(h)=A_{1}(h)+C_{2}(h)+\mathbf{E}\left\{d R_{k}\left(\mathcal{K}^{\perp} h\right) \mid \mathcal{F}^{x_{0}}\right\},
$$

where $\mathcal{K}$ is as defined by (5.1) and

$$
\begin{aligned}
C_{2}(h)= & \int_{0}^{T} \int_{0}^{s_{k+1}} \ldots \int_{0}^{s_{2}}\left\langle d\left(a_{k+1}\left(s_{1}, \ldots, s_{k+1}\right)\right)(h)\right. \\
& \left.-\mathbf{E}\left\{d\left(a_{k+1}\left(s_{1}, \ldots, s_{k+1}\right)\right)\left(\mathcal{K}^{\perp} h\right) \mid \mathcal{F}^{x_{0}}\right\}, K^{\perp}\left(x_{s_{1}}\right) d B_{s_{1}} \otimes \ldots K^{\perp}\left(x_{s_{k+1}}\right) d B_{s_{k+1}}\right\rangle .
\end{aligned}
$$

However

$\left\|C_{2}\right\|_{L^{2}}^{2} \leq 4 \int_{0}^{T} \int_{0}^{s_{k+1}} \ldots \int_{0}^{s_{2}}\left\|d a_{k+1}\left(s_{1}, \ldots, s_{k+1}\right)\right\|_{L^{2}}^{2} d s_{1} \ldots d s_{k+1}=4\left\|d a_{k+1}\right\|_{L^{2}}^{2}$

and $\left\|\mathbf{E}\left\{d R_{k}(-) \mid \mathcal{F}^{x_{0}}\right\}\right\|_{L^{2}} \leq\left\|d R_{k}(-)\right\|_{L^{2}}$ giving

$$
\left\|d\left(\mathbf{E}\left\{R_{k} \mid \mathcal{F}^{x_{0}}\right\}\right)\right\|_{L^{2}}^{2} \leq 6\left\|A_{1}\right\|_{L^{2}}^{2}+24\left\|d a_{k+1}\right\|_{L^{2}}^{2}+6 \mid d R_{k}(-) \|_{L^{2}}^{2} .
$$

For $f \in \mathbb{D}_{\mathcal{F}^{x_{0}}}^{2,1},\left\|d R_{k}(-)\right\|_{L^{2}}^{2} \rightarrow 0$ and $\left\|d a_{k+1}\right\|_{L^{2}}^{2} \rightarrow 0$ by (7.3). Thus we only need to show that $\left\|A_{1}\right\|_{L^{2}} \rightarrow 0$. Now

$$
\left\|A_{1}\right\|_{L^{2}}^{2} \leq C k^{2} \mathbf{E} \int_{0}^{T} \int_{0}^{s_{k+1}} \ldots \int_{0}^{s_{2}}\left\|a^{k+1}\left(s_{1}, \ldots, s_{k+1}\right)\right\|^{2} d s_{1} \ldots d s_{k+1} \leq C k^{2}\left\|R_{k}\right\|_{L^{2}}^{2},
$$

by Proposition 3.3, which converges to zero if $f$ belongs to $\operatorname{Dom}(\Delta)$. 
Remark 7.4 If we can show that $\left\|A_{1}\right\|_{L^{2}}^{2} \rightarrow 0$ without the condition $f \in \operatorname{Dom}(\Delta)$ we would have shown that $\mathcal{I}^{*}\left[\mathbb{D}^{2,1}\left(\mathcal{C}_{x_{0}} M ; \mathbf{R}\right)\right]=\mathbb{D}_{\mathcal{F}^{x_{0}}}^{2,1}(\Omega ; \mathbf{R})$. This convergence should hold, though we do not have a proof, as the similar term

$$
\begin{aligned}
\left\|A_{2}(h)\right\|_{L^{2}} \equiv & \| \sum_{l=1}^{k+1} \int_{0}^{T} \int_{0}^{s_{k+1}} \ldots \int_{0}^{s_{2}}\left\langle a_{k+1}\left(s_{1}, \ldots, s_{k+1}\right),\right. \\
& \left.K^{\perp}\left(x_{s_{1}}\right) d B_{s_{1}} \otimes \ldots K^{\perp}\left(x_{s_{l}}\right) \dot{h}_{s_{l}} \otimes \ldots K^{\perp}\left(x_{s_{k+1}}\right) d B_{s_{k+1}}\right\rangle \|_{L^{2}} \rightarrow 0
\end{aligned}
$$

following from $\mathbf{E}\left\{d R_{k}(h) \mid \mathcal{F}^{x_{0}}\right\} \rightarrow 0$.

Observe that the pull back by $\mathcal{I}$ of $B C^{2}$ functions belong to $\mathbb{D}_{\mathcal{F}^{x_{0}}}^{2,2}(\Omega, \mathbf{R})$ and $\mathbb{D}_{\mathcal{F}^{x_{0}}}^{2,2}(\Omega, \mathbf{R}) \subset \mathcal{I}^{*}\left[\mathbb{D}^{2,1}\left(\mathcal{C}_{x_{0}} M ; \mathbf{R}\right)\right]$ by Proposition 7.3 . We are lead to the following :

Corollary 7.5 If Condition $\left(M_{0}\right)$ holds then $B C^{2}$ functions on $\mathcal{C}_{x_{0}} M$ are in $\mathbb{D}^{2,1}\left(\mathcal{C}_{x_{0}} M ; \mathbf{R}\right)$.

Corollary 7.5 corrects Theorem 2.1 of [25] which stated that $B C^{1}$ functions are in $\mathbb{D}^{2,1}\left(\mathcal{C}_{x_{0}} M ; \mathbf{R}\right)$.

\subsection{On the uniqueness of $d$}

Definition 7.6 We say uniqueness holds for $d^{p}$ if the closure of $d^{p}$ is independent of the choice of its initial domain $d_{\mathcal{H}}$ satisfying $\operatorname{Cyl} \subset \operatorname{Dom}\left(d_{\mathcal{H}}\right) \subset B C^{1}$.

Remark 7.7 Since $B C^{1} \subset W^{p, 1}$ uniqueness for $d^{p}$ would be implied by Markov uniqueness. It would also follow more generally if the image $\mathcal{I}^{*}\left[\mathbb{D}^{p, 1}\right]$ is independent of the choice of $\operatorname{Dom}\left(d_{\mathcal{H}}\right)$.

Theorem 7.8 Assume Condition $\left(M_{0}\right)$. Suppose $T$ is a closed operator,

$$
T: \operatorname{Dom}(T) \subset L^{2}\left(\mathcal{C}_{x_{0}} M ; \mathbf{R}\right) \subset L^{2} \Gamma \mathcal{H}^{*}
$$

with the properties that

(i) T agrees with $d_{\mathcal{H}}$ on smooth cylindrical functions.

(ii) Dom $\left(T^{*}\right)$ contains all smooth cylindrical one forms.

Then $d \subset T \subset \tilde{d}$, where $\tilde{d}$ is the weak derivative.

Proof It is enough to show that $(\tilde{d})^{*} \subset T^{*}$, which follows from

$$
\tilde{d}^{*}=\overline{\left.d^{*}\right|_{\mathbb{D}^{2,1}}}=\overline{\left.d^{*}\right|_{\text {Cyl }}} \subset T^{*} .
$$

Remark 7.9 If $\mathbf{E}\left\{f \mid \mathcal{F}^{x_{0}}\right\}$ is in $\mathbb{D}^{2,1}(\Omega, \mathbf{R})$ whenever $f$ is in $\mathbb{D}^{2,1}(\Omega, \mathbf{R})$ then $\mathcal{I}^{*}\left[\mathbb{D}^{2,1}\right]=$ $\mathbb{D}_{\mathcal{F}^{x_{0}}}^{2,1}$ and so Markov uniqueness, and hence uniqueness of $d$, holds. To see this take $f$ in $\mathbb{D}_{\mathcal{F} x_{0}}^{2,1}$ so $f=\lim _{n} f^{n}$ in $\mathbb{D}^{2,1}$ when $f^{n}$ is the sum of the finite terms in the chaos expansion of $f$. We saw above that each $\mathbf{E}\left\{f^{n} \mid \mathcal{F}^{x_{0}}\right\}$ lies in $\mathcal{I}^{*}\left[\mathbb{D}^{2,1}\right]$. Since the latter is closed $f$ itself must lie in it. See the Appendix, section 10. 


\section{Covariant Differentiation}

Our main aim in this section is to define higher order Sobolev and weak Sobolev spaces and to prove the pull back theorem, Theorem 8.14.

For $G$ a separable Hilbert space we can define $W^{p, 1} G \equiv W^{p, 1}\left(\mathcal{C}_{x_{0}} M ; G\right)$ by Proposition 5.5 to be the domain, by graph norm, of the closure of $\tilde{d}^{G}$ whose domain $\operatorname{Dom}\left(\tilde{d}^{G}\right)$ consists of finite sums $\sum_{j} f_{j} g_{j}$ for $f_{j} \in W^{p, 1}\left(\mathcal{C}_{x_{0}} M ; \mathbf{R}\right), g_{j} \in G$. Let

$$
\tilde{d} \equiv \tilde{d}^{G}: W^{p, 1} G \subset L^{p}\left(\mathcal{C}_{x_{0}} M ; G\right) \rightarrow L^{p} \Gamma\left(\mathbb{L}_{2}(\mathcal{H} ; G)\right)
$$

also denote this closure.

Proposition 8.1 Assume Condition $\left(M_{0}\right)$. For $f: \mathcal{C}_{x_{0}} M \rightarrow \mathbf{R}$ the following are equivalent:

(i) $f \in W^{p, 1}\left(\mathcal{C}_{x_{0}} M ; G\right)$

(ii) $\mathcal{I}^{*} f \in \mathbb{D}^{p, 1}\left(\mathcal{C}_{0} \mathbf{R}^{m} ; G\right)$;

(iii) There is a constant $c_{f}$ such that if $\frac{1}{p}+\frac{1}{q}=1$ then for all G-valued 1-forms $\phi \in \mathbb{D}^{q, 1}\left(\mathcal{C}_{x_{0}} M ; \mathbb{L}_{2}(\mathcal{H} ; G)\right)$,

$$
\left|\int_{\mathcal{C}_{x_{0}} M}\left\langle f,\left(d^{G}\right)^{*} \phi\right\rangle_{G} d \mu_{x_{0}}\right| \leq c_{f}\|\phi\|_{L^{q} \Gamma \mathbb{L}_{2}(\mathcal{H} ; G)} .
$$

If $f \in W^{p, 1}\left(\mathcal{C}_{x_{0}} M ; G\right)$ the intertwining formula of Theorem 6.1 and (6.5) extend to $G$-valued functions.

Proof Suppose $f \in W^{p, 1} G$. Then $f=\lim _{l \rightarrow \infty} f_{l}$ in graph norm for some sequence $\left\{f_{l}\right\}_{l=1}^{\infty}$ in $G \otimes_{0} \operatorname{Dom}(\tilde{d})$. By Theorem 6.1, $\mathcal{I}^{*}\left(f_{l}\right) \in \mathbb{D}^{p, 1}$ for each $l$ and

$$
d \mathcal{I}^{*}\left(f_{l}\right)=\mathcal{I}^{*}\left(\tilde{d}^{G} f_{l}\right)
$$

By assumption $\tilde{d}^{G} f_{l} \rightarrow \tilde{d}^{G} f$ in $L^{p}$ and so by continuity of $\mathcal{I}^{*}$, Theorem $5.7, d \mathcal{I}^{*}\left(f_{l}\right)$ converges in $L^{p}$, showing that $\mathcal{I}^{*}(f) \in \mathbb{D}^{p, 1}$ and $d\left(\mathcal{I}^{*} f\right)=\mathcal{I}^{*}\left(\tilde{d}^{G} f\right)$.

Conversely, if $\mathcal{I}^{*}(f) \in \mathbb{D}^{p, 1}\left(\mathcal{C}_{0} \mathbf{R}^{m} ; G\right)$, taking an orthogonal base $\left\{g_{i}\right\}_{i=1}^{\infty}$ for $G$

$$
\mathcal{I}^{*}(f)=\lim _{k \rightarrow \infty} \sum_{l=1}^{k} \alpha_{l} g_{l}
$$

in $\mathbb{D}^{p, 1}\left(\mathcal{C}_{0} \mathbf{R}^{m} ; G\right)$ for $\alpha_{l}=\left\langle\mathcal{I}^{*}(f), g_{l}\right\rangle_{G} \in \mathbb{D}_{\mathcal{F}^{x_{0}}}^{p, 1}\left(\mathcal{C}_{0} \mathbf{R}^{m} ; \mathbf{R}\right)$. By Theorem $6.1 \alpha_{l}=$ $\mathcal{I}^{*}\left(f_{l}\right)$ some $f_{l} \in W^{p, 1}\left(\mathcal{C}_{x_{0}} M ; \mathbf{R}\right)$. Then $f=\lim _{k \rightarrow \infty} \sum_{l=1}^{k} f_{l} g_{l}$ in $L^{p}\left(\mathcal{C}_{x_{0}} M ; \mathbf{R}\right)$. Since

$$
\tilde{d}^{G}\left(\sum_{l=1}^{k} f_{l} g_{l}\right)(-)=\sum_{l=1}^{k} \tilde{d} f_{l}(-) g_{l}
$$

and $\tilde{d} f_{l}$ is given in terms of $d \alpha_{l}$ by equation (6.5), we see the convergence is in $W^{p, 1}\left(\mathcal{C}_{x_{0}} M ; G\right)$ and so $f \in W^{p, 1}\left(\mathcal{C}_{x_{0}} M ; G\right)$. Thus (i) is equivalent to (ii). That (ii) is equivalent to (iii) can be seen as for the scalar case in Theorem 6.1. The only additional point is to observe that if $U$ is defined by (5.10) then $G \otimes_{0} U$ is total in $\mathbb{D}^{p, 1}(\Omega, G \otimes H)$ and $\overline{(\mathbf{1} \otimes T \mathcal{I})(h)} \in \mathbb{D}^{p, 1}(G \otimes \mathcal{H})$ for each $h \in G \otimes_{0} U$. Thus $G \otimes_{0} U$ can take over the role $U$ played in the proof of Theorem 6.1. This proof also shows that the analogue of (6.5) holds for $G$-valued functions. 


\subsection{The pointwise and the damped Markovan connections}

To define higher order Sobolev spaces we need to introduce covariant derivatives.

Using the notation of $\S 2.3$ consider the principal $\mathcal{C}_{\text {Id }} O(p)$-bundle $C_{x_{0}} O E \rightarrow \mathcal{C}_{x_{0}} M$ and its associated Hilbert bundle $L^{2} \mathcal{E} \rightarrow \mathcal{C}_{x_{0}} M$. As described in Eliasson [14] our metric connection $\nabla$ on $E$ induces a so called pointwise connection $\bar{\nabla}$ on $L^{2} \mathcal{E}$.

$$
\bar{\nabla}_{v} U=\left.\frac{D}{\partial s} U\left(\exp _{\sigma .} s v .\right)\right|_{s=0}
$$

for $U$ a smooth section of $L^{2} \mathcal{E}$ and $v \in T_{\sigma} C_{x_{0}} M$ where $\frac{D}{\partial s}$ and exp come from $\nabla$.

The almost surely defined map $\frac{\mathbb{D}}{d}: \mathcal{H} \rightarrow L^{2} \mathcal{E}$, is an isometric isomorphism which we used to give $\mathcal{H}$ a vector bundle structure (at least over the subset on which $\frac{D}{\partial}$ is defined). We also use it to pull back $\bar{\nabla}$ to obtain a connection on $\mathcal{H}$, which we shall denote by $\mathbb{\nabla}$. By definition

$$
\mathbb{W}=\mathbf{W} \bar{\nabla} \frac{\mathbb{D}}{d t},
$$

see (2.15). As usual these connections induce connections on the relevant tensor bundles and in particular on the dual bundles $\left(L^{2} \mathcal{E}\right)^{*}$ and $\mathcal{H}^{*}$ respectively. Since the connections are metric the latter are compatible with the natural isometries

$$
\left(L^{2} \mathcal{E}\right)^{*} \rightarrow L^{2} \mathcal{E}, \quad(\mathcal{H})^{*} \rightarrow \mathcal{H}
$$

For $X: M \times \mathbf{R}^{m} \rightarrow T M$ as in (2.5) define:

$$
\bar{X}: C_{x_{0}} M \times L^{2}\left([0, T] ; \mathbf{R}^{m}\right) \rightarrow L^{2} \mathcal{E}
$$

by

$$
(\bar{X}(\sigma) h)_{t}=X(\sigma(t))(h(t)), \quad 0 \leq t \leq T
$$

and its right inverse:

$$
\begin{gathered}
\bar{Y}: L^{2} \mathcal{E} \rightarrow L^{2}\left([0, T] ; \mathbf{R}^{m}\right) \\
\bar{Y}(v)_{t}=Y_{\sigma(t)}(v(t)), \quad 0 \leq t \leq T .
\end{gathered}
$$

Also define

$$
\boldsymbol{X}: C_{x_{0}} M \times H \rightarrow \mathcal{H}
$$

by

$$
\boldsymbol{X}(\sigma)(h)=\overline{T \mathcal{I}}_{\sigma}(h) \equiv \mathbf{W} \bar{X}(\sigma)(\dot{h} .), \quad 0 \leq t \leq T
$$

with right inverse $\mathbb{Y}: \mathcal{H} \rightarrow H$, as defined by (5.1).

Proposition 8.2 The connections $\bar{\nabla}, \mathbb{\nabla}$ on $L^{2} \mathcal{E}$ and $\mathcal{H}$ are the connections corresponding to $\bar{X}$ and $\boldsymbol{X}$ respectively in the sense that $\bar{\nabla}_{v} \bar{X}(\dot{h})=0$ and $\mathbb{\nabla}_{v} \boldsymbol{X}(h)=0$ if $\dot{h} \in[\operatorname{ker} \bar{X}(\sigma)]^{\perp}$ or equivalently $h \in[\operatorname{ker} \boldsymbol{X}(\sigma)]^{\perp}$ and $v \in T_{\sigma} \mathcal{C}_{x_{0}} M$.

If $U, \frac{\mathbb{D}}{\partial t} V$ are $C^{1}$ sections of $L^{2} \mathcal{E}$ and $v \in T_{\sigma} \mathcal{C}_{x_{0}} M$ then

$$
\bar{\nabla}_{v} U=\bar{X}(\sigma) d\left(\alpha \mapsto \bar{Y}_{\alpha} U(\alpha)\right)(v)
$$

and

$$
\mathbb{\nabla}_{v} V=\boldsymbol{X}(\sigma) d\left(\alpha \mapsto \mathbb{Y}_{\alpha} V(\alpha)\right)(v)
$$


Proof By definition

$$
\begin{aligned}
\bar{\nabla}_{v} \bar{X}(\dot{h})_{t} & =\left.\frac{D}{d s} X\left(\exp _{\sigma(t)} s v_{t}\right)(\dot{h}(t))\right|_{s=0} \\
& =\nabla_{v_{t}} X(\dot{h}(t)) \\
& =0, \text { if } \dot{h}(t) \perp \operatorname{ker}[X(\sigma(t))] .
\end{aligned}
$$

However $\dot{h} \perp \operatorname{ker}[\bar{X}(\sigma)]$ in $L^{2}\left([0, T] ; \mathbf{R}^{m}\right)$ holds if and only if $\dot{h}(t)$ is orthogonal to $\operatorname{ker}[X(\sigma(t))]$ for almost all $t$, and we see the pointwise connection is the connection corresponding to $\bar{X}$. From this (8.3) holds as in Elworthy-LeJan-Li [15]. The results for $\mathcal{H}$ follow by conjugation with $\frac{\mathbb{D}}{\partial t}$.

In the case $E=T M$ the connection $\mathbb{\nabla}$ is the damped Markovian connection defined in a different way by Cruzeiro-Fang [5] and we refer to it as that in our more general situation. For the case of $M$ a Lie group with left invariant metric and connection, as Example 2 of section 2.1.1, see section 9.

\subsection{Covariant Gross-Sobolev derivatives}

Let $G$ be a separable Hilbert space. First consider a smooth separable Hilbert bundle $\mathcal{G}$ over $\mathcal{C}_{x_{0}} M$ with a metric connection $\tilde{\nabla}$ determined by a smooth surjective vector bundle map $\tilde{X}: \mathcal{C}_{x_{0}} M \times G \rightarrow \mathcal{G}$, with isometric right inverse

$$
\tilde{Y}_{\sigma}=\tilde{X}(\sigma)^{*}: \mathcal{G}_{\sigma} \rightarrow G, \quad \sigma \in \mathcal{C}_{x_{0}} M
$$

Define $\operatorname{Dom}\left(\tilde{\nabla}^{p}\right)$ to be $\mathbb{D}^{p, 1} \mathcal{G}$ for

$$
\mathbb{D}^{p, 1} \mathcal{G}=\left\{V \in L^{p} \Gamma \mathcal{G} \mid \tilde{Y} V \in \mathbb{D}^{p, 1}\left(\mathcal{C}_{x_{0}} M ; G\right)\right\}
$$

For $V \in \mathbb{D}^{p, 1} \mathcal{G}, \phi \in \mathbb{D}^{p, 1} \mathbb{L}_{2}(\mathcal{G} ; K), K$ a separable Hilbert space set

$$
\begin{aligned}
\tilde{\nabla}_{v}^{p} V & =\tilde{X}(\sigma) d^{p}(\tilde{Y} V)_{\sigma}(v), \quad v \in \mathcal{H}_{\sigma} . \\
\tilde{\nabla}_{v}^{p} \phi & =d^{p}(\phi(\tilde{X}-))_{\sigma}(v)\left(\tilde{Y}_{\sigma}-\right) .
\end{aligned}
$$

Let $\tilde{K}^{\perp}(\sigma)=\tilde{Y}_{\sigma} \tilde{X}(\sigma)$ be the orthogonal projection of $G$ onto $\operatorname{ker}[\tilde{X}(\sigma)]^{\perp}, \sigma \in$ $\mathcal{C}_{x_{0}} M$. Note that, c.f. [15]:

$$
\tilde{X} \tilde{\nabla} \tilde{Y}=0, \quad \text { and } \quad \tilde{X} d_{\mathcal{H}} \tilde{K}^{\perp}=0 .
$$

To obtain a closed covariant differentiation operator we impose:

Condition $K^{p}$. If $f \in \mathbb{D}^{p, 1}\left(\mathcal{C}_{x_{0}} M ; G\right)$ then $\tilde{K}^{\perp}(\cdot) f(\cdot) \in \mathbb{D}^{p, 1}\left(\mathcal{C}_{x_{0}} M ; G\right)$.

Note that this implies, by the closed graph theorem, that $f \mapsto \tilde{K}^{\perp} f$ is continuous from $\mathbb{D}^{p, 1}\left(\mathcal{C}_{x_{0}} M ; G\right)$ to $\mathbb{D}^{p, 1}\left(\mathcal{C}_{x_{0}} M ; G\right)$. Note that if $\tilde{X}=\bar{X}$ or $\boldsymbol{X}$, then Condition $\left(M_{0}\right)$ implies Condition $K^{p}$ holds for all $p$ by Lemma 5.6.

Proposition 8.3 Assume Condition $K^{p}$. Then $C^{\infty} \Gamma \mathcal{G} \cap \mathbb{D}^{p, 1} \mathcal{G}$ is dense in $\mathbb{D}^{p, 1} \mathcal{G}$.

Proof For $V \in \mathbb{D}^{p, 1} \mathcal{G}$ set $f=\tilde{Y} V$ and take smooth functions $f_{k} \in \mathbb{D}^{p, 1}\left(\mathcal{C}_{x_{0}} M ; G\right)$, $k=1,2, \ldots$, converging to $f$ in $\mathbb{D}^{p, 1}\left(\mathcal{C}_{x_{0}} M ; G\right)$. Then $\tilde{X} f_{k} \rightarrow V$ in $L^{p}$. Observe that

$$
\tilde{\nabla}\left(\tilde{X} f_{k}\right)=\tilde{X} d_{\mathcal{H}}\left(\tilde{Y} \tilde{X} f_{k}\right) \rightarrow \tilde{X} d(\tilde{Y} \tilde{X} f)=\tilde{\nabla} V
$$


in $L^{p}$ by Condition $K^{p}$.

The proposition is essentially due to the fact that Condition $K^{p}$ implies the smoothness of $\tilde{X}$ and in particular

$$
U \in \mathbb{D}^{p, 1}\left(\mathcal{C}_{x_{0}} M ; G\right) \Longleftrightarrow \tilde{X}(U) \in \mathbb{D}^{p, 1} \mathcal{G} .
$$

Corollary 8.4 Assume Condition $K^{p}$. There is the Leibniz formula

$$
d^{p}(\tilde{Y} V)=\left(\tilde{\nabla}_{-} \tilde{Y}\right) V+\tilde{Y} \tilde{\nabla}_{-}^{p} V, \quad V \in \mathbb{D}^{p, 1} \mathcal{G} .
$$

Proof The formula holds for smooth $V$ and so in general by Proposition 8.3 since $d^{p}$ is closed.

Lemma 8.5 Assume Condition $K^{p}$. Then for $1<p<\infty$, and for $1 \leq p<\infty$ if condition $\left(M_{0}\right)$ holds,

$$
\tilde{\nabla}^{p}: \operatorname{Dom}\left(\tilde{\nabla}^{p}\right) \subset L^{p} \Gamma \mathcal{G} \rightarrow L^{p} \Gamma\left(\mathbb{L}_{2}(\mathcal{H} ; \mathcal{G})\right)
$$

is a closed operator.

Proof Let $\left\{U^{k}\right\}_{k=0}^{\infty}$ be a sequence in $\mathbb{D}^{p, 1} \mathcal{G}$ such that $\left\{U^{k}, \tilde{\nabla}^{p} U^{k}\right\}_{k=0}^{\infty}$ converges to some $(U, Z)$. We must show that $U \in \mathbb{D}^{p, 1} \mathcal{G}$ and $Z=\tilde{\nabla}^{p} U$. By definition $\tilde{Y}\left(U^{k}\right) \in$ $\mathbb{D}^{p, 1}\left(\mathcal{C}_{x_{0}} M ; G\right)$ and $\tilde{Y}\left(U^{k}\right) \rightarrow \tilde{Y}(U)$. Apply the Leibniz formula to see that

$$
d\left(\tilde{Y} U^{k}\right)=\left(d_{\mathcal{H}} \tilde{K}^{\perp}\right)\left(\tilde{Y} U^{k}\right)+\tilde{Y} \tilde{\nabla}^{p} U^{k} \rightarrow\left(d_{\mathcal{H}} \tilde{K}^{\perp}\right)(\tilde{Y} U)+\tilde{Y} Z
$$

The convergence is in $L^{p} \Gamma \mathbb{L}_{2}(\mathcal{H} ; G)$ by Condition $K^{p}$. Since $d$ is closed this shows that $\tilde{Y} U \in \mathbb{D}^{p, 1}\left(\mathcal{C}_{x_{0}} M ; G\right)$ and $\lim _{k \rightarrow \infty} d\left(\tilde{Y} U^{k}\right)=d(\tilde{Y} U)$. Consequently $U \in \mathbb{D}^{p, 1} \mathcal{G}$ and, using (8.5), $\tilde{\nabla}^{p} U^{k}=\tilde{X} d\left(\tilde{Y} U^{k}\right) \rightarrow \tilde{X} d(\tilde{Y} U)=\tilde{\nabla}^{p} U$, giving $Z=\tilde{\nabla}^{p} U$.

Note that if $\mathcal{G}^{1}$ and $\mathcal{G}^{2}$ are smooth Hilbert bundles over $\mathcal{C}_{x_{0}} M$ with metric connections given respectively by

$$
\tilde{X}^{j}: \mathcal{C}_{x_{0}} M \times G^{j} \rightarrow \mathcal{G}^{j}, j=1,2
$$

then the natural induced metric connections on the Hilbert bundles $\left(\mathcal{G}^{1}\right)^{*}, \mathcal{G}^{1} \otimes \mathcal{G}^{2}$, $\mathbb{L}_{2}\left(\mathcal{G}^{1} ; \mathcal{G}^{2}\right)$ are determined by

$$
\begin{aligned}
& \mathcal{C}_{x_{0}} M \times\left(G^{1}\right)^{*} \rightarrow\left(\mathcal{G}^{1}\right)^{*} \\
&(\sigma, l) \mapsto l \circ Y_{\sigma}, \\
& \tilde{X}^{1} \otimes \tilde{X}^{2}: \mathcal{C}_{x_{0}} M \times\left(G^{1} \otimes G^{2}\right) \rightarrow \mathcal{G}^{1} \otimes \mathcal{G}^{2}
\end{aligned}
$$

and

$$
\begin{array}{rlr}
\mathcal{C}_{x_{0}} M \times \mathbb{L}_{2}\left(G^{1} ; G^{2}\right) & \rightarrow & \mathbb{L}_{2}\left(\mathcal{G}^{1} ; \mathcal{G}^{2}\right) \\
(\sigma, T) & \mapsto & \tilde{X}^{2}(\sigma) T \tilde{Y}_{\sigma}^{1}
\end{array}
$$

respectively. Using these, iteratively, we can obtain closed operators acting on sections of the tensor bundles constructed from an initial Hilbert bundle $\mathcal{G}$, given the relevant 
Condition $K^{p}$. For 'bundles' of the form $\mathbb{L}_{2}\left(\mathcal{H} ; \mathbb{L}_{2}(\mathcal{H} ; \mathcal{G})\right)$ which we can more compactly write as $\mathcal{G} \otimes\left(\mathcal{H}^{\otimes^{2}}\right)^{*}$ we will use the isometry $\frac{\mathbf{D}}{\partial t}: \mathcal{H} \rightarrow L^{2} \mathcal{E}$ to pull back the covariant derivative operator from the one obtained as above on $\mathcal{G} \otimes\left(\left(L^{2} \mathcal{E}\right)^{\otimes^{2}}\right)^{*}$, (in this case) using the pointwise connection on $L^{2} \mathcal{E}$. For example $\mathbb{D}^{p, 1}\left(\mathcal{G} \otimes\left(\mathcal{H}^{\otimes^{2}}\right)\right)$ is

$$
\left\{V \in L^{p}\left(\mathcal{G} \otimes\left(\mathcal{H}^{\otimes^{2}}\right)\right) \mid\left(\mathbf{1} \otimes \frac{\mathbb{D}}{\partial t} \otimes \frac{\mathbb{D}}{\partial t}\right) V \in \mathbb{D}^{p, 1}\left(\mathcal{G} \otimes\left(L^{2} \mathcal{E}\right)^{\otimes 2}\right)\right\} .
$$

Theorem 8.6 Assume Condition $K^{p}$ for $\bar{X}$ for all $1<p<\infty$. Then for $1<p<$ $\infty$ the above construction yields closed covariant derivative operators with domain $\operatorname{Dom}\left(\mathbb{\nabla}^{p}\right)$ :

$$
\begin{aligned}
\mathbb{\nabla}^{p}: \quad & L^{p} \Gamma\left(\left(L^{2} \mathcal{E}\right)^{\otimes r} \otimes\left(L^{2} \mathcal{E}\right)^{\otimes s} \otimes \mathcal{H}^{\otimes a} \otimes\left(\mathcal{H}^{*}\right)^{\otimes b}\right) \\
& \longrightarrow L^{p} \Gamma\left(\left(L^{2} \mathcal{E}\right)^{\otimes r} \otimes\left(L^{2} \mathcal{E}\right)^{\otimes s} \otimes \mathcal{H}^{\otimes a} \otimes\left(\mathcal{H}^{*}\right)^{\otimes b+1}\right)
\end{aligned}
$$

for any $r, s, a, b, \in\{0,1,2, \ldots$,$\} . Moreover V \in \operatorname{Dom}\left(\mathbb{\nabla}^{p}\right)$ if and only if

$$
\begin{aligned}
X^{r, s, a, b} V & \equiv\left(\left(\otimes^{r} \bar{X}\right) \otimes\left(\circ\left(\otimes^{s} \bar{Y}\right)\right) \otimes\left(\otimes^{a} \boldsymbol{X}\right) \otimes\left(\circ\left(\otimes^{b} \mathbb{Y}\right)\right)\right) V: \\
\mathcal{C}_{x_{0}} M & \longrightarrow\left(L ^ { 2 } ( [ 0 , T ] ; \mathbf { R } ^ { m } ) ^ { \otimes ^ { r } } \otimes \left(L^{2}\left([0, T] ; \mathbf{R}^{m}\right)^{\otimes^{s}} \otimes H^{\otimes^{a}} \otimes\left(H^{*}\right)^{\otimes^{b}}\right.\right.
\end{aligned}
$$

is in $\mathbb{D}^{p, 1}$ and then $\mathbb{\nabla}^{p}=\left(X^{r, s, a, b}\right)^{*} d^{p}\left(X^{r, s, a, b} V\right)$. If Condition $\left(M_{0}\right)$ holds we may take $p=1$.

Proof It is only necessary to observe that Condition $K^{p}$ for $X^{r, s, a, b}$ is implied by Condition $K^{q}$ for all $1<q<\infty$ for $\bar{X}$, and if Condition $\left(M_{0}\right)$ holds then $X^{r, s, a, b}$ satisfies $K^{1}$ by Lemma 5.6.

\subsection{The higher order Sobolev spaces $\mathbb{D}^{p, k}$}

Suppose that $\mathcal{G}$ is a smooth Hilbert bundle over $\mathcal{C}_{x_{0}} M$ with connection $\tilde{\nabla}$ as given in $\S 8.2$ by some $\tilde{X}$ which together with all tensor products

$$
\tilde{X} \otimes\left(\otimes^{a} \bar{X}\right) \otimes\left(\circ \otimes^{b} \bar{Y}\right)
$$

satisfies Condition $K^{p}$. For $a, b \in\{0,1,2, \ldots\}$ we can inductively define $\mathbb{\nabla}^{p,(k)}$ and $\mathbb{D}^{p, k}\left(\mathcal{G} \otimes \mathcal{H}^{\otimes a} \otimes\left(\mathcal{H}^{*}\right)^{\otimes b}\right), k=1,2, \ldots$ as follows:

Set $\mathbb{\nabla}^{p,(1)}=\mathbb{\nabla}^{p}$, defined as in Theorem 8.6, with

$$
\mathbb{D}^{p, 1}\left(\mathcal{G} \otimes \mathcal{H}^{\otimes a} \otimes\left(\mathcal{H}^{*}\right)^{\otimes b}\right)=\operatorname{Dom}\left(\mathbb{W}^{p}\right) .
$$

For $k=\{2,3, \ldots\}$ set

$\mathbb{D}^{p, k}\left(\mathcal{G} \otimes \mathcal{H}^{\otimes a} \otimes\left(\mathcal{H}^{*}\right)^{\otimes b}\right)=\left\{V \in \mathbb{D}^{p, 1} \mid \mathbb{\nabla}^{p} V \in \mathbb{D}^{p, k-1}\left(\mathcal{G} \otimes \mathcal{H}^{\otimes a} \otimes\left(\mathcal{H}^{*}\right)^{\otimes(b+1)}\right)\right\}$

and $\mathbb{\nabla}^{p,(k)}=\mathbb{\nabla}^{p,(k-1)} \circ \mathbb{\nabla}^{p}$. Here we have used our usual identification of $\mathbb{L}_{2}\left(H_{1} ; H_{2}\right)$ with $H_{2} \otimes H_{1}^{*}$ for Hilbert spaces $H_{1}, H_{2}$. As usual we give $\mathbb{D}^{p, k}$ the graph norm

$$
|V|_{p, k}=\left(\left.\left\|\left.V\right|_{L^{p}} ^{p}+\right\| \mathbb{W}^{p} V\right|_{L^{p}} ^{p}+\ldots+\|\left.\mathbb{W}^{p,(k)} V\right|_{L^{p}} ^{p}\right)^{\frac{1}{p}}
$$

i.e. the graph of the closed operator $\operatorname{Id} \otimes \mathbb{W}^{p} \oplus \ldots \oplus \mathbb{\nabla}^{p,(k)}$. 


\subsubsection{The higher order weak Sobolev spaces $W^{p, k}$}

Continuing with the previous notation let

$$
\begin{aligned}
\left(\mathbb{\mathbb { W }}^{p}\right)^{*}: \operatorname{Dom}\left(\mathbb{\nabla}^{p}\right)^{*} & \subset \quad L^{q} \Gamma\left(\mathcal{G} \otimes \mathcal{H}^{\otimes r} \otimes \mathcal{H}^{* \otimes s+1}\right) \\
& \longrightarrow \quad L^{q} \Gamma\left(\mathcal{G} \otimes \mathcal{H}^{\otimes r} \otimes \mathcal{H}^{* \otimes s}\right)
\end{aligned}
$$

be the adjoint of $\mathbb{\nabla}^{p}, \frac{1}{p}+\frac{1}{q}=1$, with $q=\infty$ if $p=1$.

Lemma 8.7 Assume Condition $K^{p}$ holds for $\tilde{X}^{r, s}$ and $\tilde{\boldsymbol{X}}^{r, s}$ where

$$
\begin{aligned}
\tilde{X}^{r, s} & =\tilde{X} \otimes\left(\otimes^{r} \bar{X}\right) \otimes\left(\circ\left(\otimes^{s} \bar{Y}\right)\right), \\
\tilde{\boldsymbol{X}}^{r, s} & =\tilde{X} \otimes\left(\otimes^{r} \boldsymbol{X}\right) \otimes\left(\circ\left(\otimes^{s} \mathbb{Y}\right) .\right.
\end{aligned}
$$

Then

(i) $\left(\mathbb{W}^{p}\right)^{*}=\tilde{\boldsymbol{X}}^{r, s}\left(d^{p}\right)^{*}\left(\tilde{\boldsymbol{X}}^{r, s+1}\right)^{*}$.

(ii) $\mathbb{D}^{q, 1}\left(\mathcal{G} \otimes \mathcal{H}^{\otimes r} \otimes \mathcal{H}^{* \otimes s+1}\right) \subset \operatorname{Dom}\left(\mathbb{\nabla}^{p}\right)^{*}$

Proof After conjugation with $\frac{\mathbb{D}}{d t}$ if necessary we can assume that $r=s=0$. Then by (8.5), $\tilde{\nabla}^{p}(-)=\tilde{X} d^{p} \tilde{Y}(-)$ and it is easy to see that

$$
\left(\tilde{\nabla}^{p}\right)^{*} \supset \tilde{X}\left(d^{p}\right)^{*}(\tilde{Y} \otimes 1)=\tilde{X}\left(d^{p}\right)^{*}(\tilde{Y} \circ-) .
$$

Suppose that $\phi \in \operatorname{Dom}\left(\tilde{\nabla}^{p}\right)^{*} \subset L^{q} \Gamma\left(\mathcal{G} \otimes \mathcal{H}^{*}\right) \sim L^{q} \Gamma \mathbb{L}_{2}(\mathcal{H} ; \mathcal{G})$. For (i) it suffices to show that $\tilde{Y} \circ \phi \in \operatorname{Dom}\left(\left(d^{p}\right)^{*}\right)$. For this take $g \in \mathbb{D}^{p, 1}\left(\mathcal{C}_{x_{0}} M ; G\right)$ then

$$
\begin{aligned}
& \left|\int_{\mathcal{C}_{x_{0}} M}\left\langle\tilde{Y} \circ \phi, d^{p} g\right\rangle d \mu_{x_{0}}\right| \\
= & \left|\int_{\mathcal{C}_{x_{0}} M}\left\langle\phi, \tilde{X} d^{p}\left(\tilde{K}^{\perp} g\right)\right\rangle d \mu_{x_{0}}\right|+\left|\int_{\mathcal{C}_{x_{0}} M}\left\langle\phi, \tilde{X} d^{p}(\tilde{K} g)\right\rangle d \mu_{x_{0}}\right| .
\end{aligned}
$$

Now $\tilde{K}^{\perp} g=\tilde{Y}(\tilde{X} g)$ and $\tilde{X} g \in \mathbb{D}^{p, 1} \mathcal{G}$. So the first of these two terms is

$$
\left|\int_{\mathcal{C}_{x_{0}} M}\left\langle\phi, \tilde{\nabla}^{p}(\tilde{X} g)\right\rangle d \mu_{x_{0}}\right| \leq c_{\phi}|\tilde{X} g|_{L^{p}} \leq \text { const. } \cdot \mathrm{c}_{\phi}|\mathrm{g}|_{\mathrm{L}^{\mathrm{p}}} .
$$

The second term is

$$
\begin{aligned}
\left|\int_{\mathcal{C}_{x_{0}} M}\left\langle\tilde{Y} \phi, d^{p}(\tilde{K} g)\right\rangle d \mu_{x_{0}}\right| & =\left|\int_{\mathcal{C}_{x_{0}} M}\left\langle\tilde{Y} \phi, d^{p}(\tilde{K})(-) g+\tilde{K} d^{p} g\right\rangle d \mu_{x_{0}}\right| \\
& \leq \mid \text { const. }\left.\left.\phi\right|_{\mathbf{L}^{\mathrm{q}} \mid \mathrm{g}}\right|_{\mathrm{L}^{\mathrm{p}}} .
\end{aligned}
$$

For (ii) suppose $\phi \in \mathbb{D}^{q, 1} \mathbb{L}_{2}(\mathcal{H} ; \mathcal{G})$. By definition,

$$
\tilde{Y} \circ \phi \in \mathbb{D}^{q, 1}\left(\mathbb{L}_{2}(\mathcal{H} ; G)\right) \subset \operatorname{Dom}\left(d^{p}\right)^{*}
$$

just as in the scalar case, Theorem 5.8. However (the easy part of ) (i) then shows $\phi \in \operatorname{Dom}\left(\tilde{\nabla}^{p}\right)^{*}$ as required. 
Define $W^{p, 1}\left(\mathcal{G} \otimes \mathcal{H}^{\otimes r} \otimes \mathcal{H}^{* \otimes s}\right)$ to be domain of the adjoint of the restriction of $\left(\mathbb{W}^{p}\right)^{*}$ to $\mathbb{D}^{q, 1}\left(\mathcal{G} \otimes \mathcal{H}^{\otimes r} \otimes \mathcal{H}^{* \otimes s+1}\right)$. Let

$$
\begin{aligned}
\tilde{\mathbb{V}}^{p}: W^{p, 1}\left(\mathcal{G} \otimes \mathcal{H}^{\otimes r} \otimes \mathcal{H}^{* \otimes s}\right) & \subset \quad L^{p} \Gamma\left(\mathcal{G} \otimes \mathcal{H}^{\otimes r} \otimes \mathcal{H}^{* \otimes s}\right) \\
& \longrightarrow \quad L^{p} \Gamma\left(\mathcal{G} \otimes \mathcal{H}^{\otimes r} \otimes \mathcal{H}^{* \otimes s+1}\right)
\end{aligned}
$$

be the adjoint, considered as a closed operator.

As for $\mathbb{D}^{p, k}$ we can define $W^{p, k}$ and $\tilde{\mathbb{W}}^{p,(k)}$ iteratively, giving $W^{p, k}$ the analogous graph norm. Since $\mathbb{\nabla}^{p,(k)} \subset \tilde{\mathbb{V}}^{p,(k)}$ we see $\mathbb{D}^{p, k}$ is always a closed subspace of $W^{p, k}$. When $\mathcal{G}$ is a trivial vector bundle $C_{x_{0}} M \times G$ we write them as $W^{p, k}\left(\mathcal{C}_{x_{0}} M ; G\right)$ and $\mathbb{D}^{p, k}\left(\mathcal{C}_{x_{0}} M ; G\right)$ respectively. By Proposition 8.1 this agrees with the previous definition when $k=1$.

We will consider the following possible conditions on $\tilde{X}$ :

Condition $\mathbf{K}(\mathbf{N})$. For $1 \leq k \leq N$, for $g \in G$, the $k^{t h} \mathcal{H}$-derivative of $\tilde{K}^{\perp}(-)(g)$ : $\mathcal{C}_{x_{0}} M \rightarrow G$ has a bound

$$
\left|\left(\mathbb{\nabla}^{\otimes(k-1)} d_{\mathcal{H}} \tilde{K}^{\perp}(-)(g)\right)\right|_{G \otimes\left(\mathcal{H}^{*}\right)^{\otimes(k-1)}}<c_{N}(\sigma)|g|_{G},
$$

where $\bar{c}_{N}=\operatorname{ess} \sup _{\sigma \in \mathcal{C}_{x_{0}} M} c_{N}(\sigma)$ is finite.

Condition K(N)D. For each $g \in G$ the map $\tilde{K}^{\perp}(-)(g)$ is in $\cap_{p>1} \cap_{k=1}^{N} \mathbb{D}^{p, k}$ and Condition $K(N)$ holds.

Remark 8.8 If Condition $M$ holds then Condition $K(N) \mathbb{D}$ holds for $\bar{X}$ and $\boldsymbol{X}$. To see this observe that Condition $K(N)$ holds because Condition $(M)$ implies that the norm $\left\|i_{\sigma}\right\|_{\sigma}$ of the inclusion $i_{\sigma}: \mathcal{H}_{\sigma} \rightarrow T_{\sigma} \mathcal{C}_{x_{0}} M$ is in $L^{\infty}\left(\mathcal{C}_{x_{0}} M ; \mathbf{R}\right)$ while all the derivatives of $K^{\perp}: M \rightarrow \mathbb{L}\left(\mathbf{R}^{m} ; \mathbf{R}^{m}\right)$ are bounded. Then Condition $K(N) D$ follows as in the proof of Lemma 5.6. Condition $\left(M_{0}\right)$ suffices for Condition $K(1) D$.

Remark 8.9 The necessity of imposing Condition $K(N) D$ in order to discuss $\mathbb{D}^{p, k}$ sections of $\mathcal{G}$ reflects the fact that we have not shown that Fréchet $B C^{\infty}$ functions are in $\cap_{p>1} \cap_{k=1}^{\infty} \mathbb{D}^{p, k}$, and $\mathcal{G}$ should be "of class $\mathbb{D}^{p, k}$ " in some sense.

Lemma 8.10 Assume Condition $\left(M_{0}\right)$. Let $1 \leq p<\infty$.

(i) Under Condition $K(N) \mathbb{D}$ the map

$$
f \mapsto \tilde{K}^{\perp} f
$$

gives a continuous linear map

$$
\mathbb{D}^{p, k}\left(\mathcal{C}_{x_{0}} M ; G\right) \rightarrow \mathbb{D}^{p, k}\left(\mathcal{C}_{x_{0}} M ; G\right)
$$

for $k \in\{1, \ldots, N\}$ and $f \mapsto d_{\mathcal{H}} \tilde{K}^{\perp}(-) f$ a continuous linear map

$$
\mathbb{D}^{p, k-1}\left(\mathcal{C}_{x_{0}} M ; G\right) \rightarrow \mathbb{D}^{p, k-1}\left(\mathcal{C}_{x_{0}} M ; \mathbb{L}_{2}(\mathcal{H} ; G)\right)
$$

for $k \in\{1,2, \ldots, N\}$. 
(ii) Under Condition $K(N)$ the corresponding results hold for weak derivatives.

Proof Assume Condition $K(N)$. Consider $\mathcal{I}^{*}\left(\tilde{K}^{\perp}(\cdot) f(\cdot)\right)$ to see

$$
\tilde{K}^{\perp}(\cdot) f(\cdot) \in W^{p, 1}\left(\mathcal{C}_{x_{0}} M ; G\right)
$$

and

$$
\tilde{d}^{p}\left(\tilde{K}^{\perp} f\right)=d_{\mathcal{H}}\left(\tilde{K}^{\perp}\right)(-) f+\tilde{K}^{\perp}(\cdot) \tilde{d}^{p} f
$$

by Proposition 8.1. Repeat this for $d_{\mathcal{H}}\left(\tilde{K}^{\perp}\right)(\tilde{X}-) f$ and higher derivatives to prove (ii) (The continuity comes from the closed graph theorem). For (i) assume $\mathrm{K}(\mathrm{N}) \mathbb{D}$. We already have the result for the weak derivatives. If $f \in \operatorname{Dom}\left(d_{\mathcal{H}}\right)^{G}$, i.e. $f \in \operatorname{Cyl} \otimes G$ we see $\tilde{K}^{\perp} f \in \mathbb{D}^{p, 1}$, as do successive derivatives of $\tilde{K}^{\perp}(-) f$. Since $\mathbb{D}^{p, 1}$ is closed in $W^{p, 1}$ we obtain the result for $k=1$, and by iterating this the result for $1 \leq k \leq N$ as required.

Proposition 8.11 Suppose $1<p<\infty$ and $k \in\{1,2, \ldots\}$. Assume Condition $\left(M_{0}\right)$ and that $\tilde{\boldsymbol{X}}^{r, s}$ defined in Lemma 8.7 satisfies Condition $K(k)$. Then

$$
V \in W^{p, k}\left(\mathcal{G} \otimes \mathcal{H}^{\otimes r} \otimes \mathcal{H}^{* \otimes s}\right)
$$

if and only if $\left(\tilde{\boldsymbol{X}}^{r, s}\right)^{*} V \in \mathbf{W}^{p, k}\left(\mathcal{C}_{x_{0}} M ; G \otimes H^{\otimes r} \otimes H^{* \otimes s}\right)$. Furthermore $\tilde{\mathbb{V}}^{p,(k)}=$ $\tilde{\boldsymbol{X}}^{r, s} \tilde{d}^{(k)}\left(\tilde{\boldsymbol{X}}^{r, s}\right)^{*} V$. The corresponding result holds for $\mathbb{D}^{p, k}$ assuming Condition $K(k) \mathbb{D}$.

Proof After conjugation by $\frac{\mathbb{D}}{d t}$ we can assume that $r=s=0$. Suppose $V \in W^{p, 1} \mathcal{G}$. Then there exists $c_{V}$ such that

$$
\left|\int_{\mathcal{C}_{x_{0}} M}\left\langle V,\left(\tilde{\nabla}^{q}\right)^{*} Z\right\rangle_{\sigma} d \mu_{x_{0}}\right| \leq c_{V}|Z|_{L^{q}}, \quad \forall Z \in \mathbb{D}^{q, 1}\left(\mathcal{G} \otimes \mathcal{H}^{*}\right) .
$$

On the other hand suppose $\phi \in \mathbb{D}^{q, 1}\left(G \otimes \mathcal{H}^{*}\right) \sim \mathbb{D}^{q, 1} \mathbb{L}_{2}(\mathcal{H} ; G)$. Then, by definition, $\tilde{X} \circ \phi \in \mathbb{D}^{q, 1} \mathbb{L}(\mathcal{H} ; \mathcal{G}) \sim \mathbb{D}^{q, 1}\left(\mathcal{G} \otimes \mathcal{H}^{*}\right)$ and so

$$
\begin{aligned}
& \left|\int_{\mathcal{C}_{x_{0}} M}\left\langle\tilde{Y} V,\left(d^{q}\right)^{*} \phi\right\rangle_{\sigma} d \mu_{x_{0}}(\sigma)\right|=\left|\int_{\mathcal{C}_{x_{0}} M}\left\langle V, \tilde{X}\left(d^{q}\right)^{*} \phi\right\rangle_{\sigma} d \mu_{x_{0}}(\sigma)\right| \\
\leq & \left|\int_{\mathcal{C}_{x_{0}} M}\left\langle V, \tilde{X}\left(d^{q}\right)^{*} \tilde{K}{ }^{\perp} \phi\right\rangle_{\sigma} d \mu_{x_{0}}(\sigma)\right|+\left|\int_{\mathcal{C}_{x_{0}} M}\left\langle V, \tilde{X}\left(d^{q}\right)^{*} \tilde{K} \phi\right\rangle_{\sigma} d \mu_{x_{0}}(\sigma)\right| .
\end{aligned}
$$

Take $Z=\tilde{X} \circ \phi$ and use Lemma 8.10 with equation (8.10) to bound the first of these two terms by a constant times $|\phi|_{L^{q}}$. To obtain a similar bound for the second term observe that there is a constant such that

$$
\left|\int_{\mathcal{C}_{x_{0}} M}\left\langle U, \tilde{X}\left(d^{G}\right)^{*} \tilde{K} \phi\right\rangle d \mu_{x_{0}}\right| \leq c|\phi|_{L^{q}}, \quad \forall U \in L^{p} \Gamma(\mathcal{G}),
$$

because if $U \in \mathbb{D}^{p, 1}$, a dense subset of $L^{p}$, we have $\tilde{Y} U \in \mathbb{D}^{p, 1}$ and

$$
\begin{aligned}
\left|\int_{\mathcal{C}_{x_{0}} M}\left\langle d^{q}(\tilde{Y} U), \tilde{K} \phi\right\rangle d \mu_{x_{0}}\right| & =\left|\int_{\mathcal{C}_{x_{0}} M}\left\langle\left(\tilde{\nabla}_{-} \tilde{Y}\right) U+\tilde{Y} \tilde{\nabla}_{-} U, \tilde{K} \phi\right\rangle d \mu_{x_{0}}\right| \\
& \leq \bar{c}_{1}|U|_{L^{p}}|\phi|_{L^{q}}
\end{aligned}
$$


by Condition $K(k)$, and since $\tilde{K} \tilde{Y}=0$. This proves that $V \in W^{p, 1} \mathcal{G}$ implies $\tilde{Y} V \in$ $W^{p, 1}\left(\mathcal{C}_{x_{0}} M ; G\right)$.

Conversely suppose $\tilde{Y} V \in W^{p, 1}\left(\mathcal{C}_{x_{0}} M ; G\right)$ and $Z \in \mathbb{D}^{q, 1}\left(\mathbb{L}_{2}(\mathcal{H} ; \mathcal{G})\right)$. We shall show (8.10) holds. Observe $\tilde{Y} \circ Z \in \mathbb{D}^{q, 1}\left(\mathbb{L}_{2}(\mathcal{H} ; G)\right)$. By Lemma 8.7,

$$
\begin{aligned}
\left|\int_{\mathcal{C}_{x_{0}} M}\left\langle V,\left(\tilde{\nabla}^{p}\right)^{*} Z\right\rangle_{\sigma} d \mu_{x_{0}}(\sigma)\right| & =\left|\int_{\mathcal{C}_{x_{0}} M}\left\langle V, \tilde{X}\left(d^{p}\right)^{*}(\tilde{Y} \circ Z)\right\rangle_{\sigma} d \mu_{x_{0}}(\sigma)\right| \\
& =\left|\int_{\mathcal{C}_{x_{0}} M}\left\langle\tilde{Y} V,\left(d^{p}\right)^{*}(\tilde{Y} \circ Z)\right\rangle_{\sigma} d \mu_{x_{0}}(\sigma)\right| \\
& \leq C_{\tilde{Y} V}|\tilde{Y} \circ Z|_{L^{q}} \leq C_{\tilde{Y} V}|\tilde{Z}|_{L^{q}} .
\end{aligned}
$$

So $V \in W^{p, 1} \mathcal{G}$ and the result holds for $k=1$.

Suppose now that the proposition holds for $W^{p, k-1}$ some $k \in\{2,3, \ldots\}$. Take $V \in W^{p, k} \mathcal{G}$. Then $V \in W^{p, k-1} \mathcal{G}$ and $\tilde{\mathbb{W}}^{p} V \in W^{p, k-1} \mathcal{G}$. Equivalently $\tilde{Y} V \in$ $W^{p, 1}\left(\mathcal{C}_{x_{0}} M ; G\right)$ and $\tilde{Y} \tilde{\mathbb{W}}^{p} V \in W^{p, k-1} \mathbb{L}_{2}(\mathcal{H} ; G)$. Now

$\tilde{d}(\tilde{Y} V)=\tilde{d}\left(\tilde{K}^{\perp} \tilde{Y} V\right)=\left(d_{\mathcal{H}} \tilde{K}^{\perp}\right)(-) \tilde{Y} V+\tilde{K}^{\perp} \tilde{d}(\tilde{Y} V)=\left(d_{\mathcal{H}} \tilde{K}^{\perp}\right)(-) \tilde{Y} V+\tilde{Y} \tilde{\nabla}^{p} V$,

which belongs to $W^{p, k-1}\left(\mathcal{C}_{x_{0}} M ; G\right)$ by the previous lemma. Thus $V \in W^{p, k} \mathcal{G}$ implies $\tilde{Y} V \in W^{p, k}\left(\mathcal{C}_{x_{0}} M ; G\right)$.

Conversely if $\tilde{Y} V \in W^{p, k}$ then

$$
\tilde{Y} \tilde{\mathbb{W}}^{p} V=\tilde{d}(\tilde{Y} V)-\tilde{\mathbb{W}}_{-}^{p} \tilde{Y}(V)=\tilde{d}(\tilde{Y} V)-\left(d_{\mathcal{H}} \tilde{K}^{\perp}\right)(-) \tilde{Y} V
$$

is in $W^{p, k-1}$ by the previous lemma, and so by the induction hypothesis $V \in W^{p, k} \mathcal{G}$.

Note that we have not discussed the independence of $\mathbb{D}^{p, k} \mathcal{G}$ from the choice of $\tilde{X}$. However our main interest is in sections of $L^{2} \mathcal{E}$ or $\mathcal{H}$ and related tensor bundles. For these we used $\tilde{X}$ derived from a stochastic differential equation and for such $\tilde{X}$ this is clear, as in Lemma 5.6. An extension of Proposition 8.3 to $\mathbb{D}^{p, k}$ and of the discussion in section 7 to prove that $B C^{\infty}$ functions are in $\cap_{p>1} \cap_{k=1}^{\infty} \mathbb{D}^{p, k}$, c.f. Remark 8.9, would give the general case.

As for the proof of Theorem 5.8, we have, using Remark 8.8

Theorem 8.12 Assume Condition $\left(M_{0}\right)$. For $1<p<\infty$, the set $W^{p, 1} \mathcal{H}$ is contained in $\operatorname{Dom}\left(\operatorname{div}^{p}\right)$ and $\operatorname{div}^{p}: W^{p, 1} \mathcal{H} \rightarrow L^{p}\left(\mathcal{C}_{x_{0}} M ; \mathbf{R}\right)$ is continuous.

\subsection{Intertwining of higher order derivatives}

First we define the Sobolev spaces over the Wiener space relative to the Itô map.

Definition 8.13 For any sub $\sigma$-algebra $\mathfrak{a}$ of the Borel $\sigma$-algebra of $\mathbf{R}^{m}$ and separable Hilbert space $G$, the space $\mathbb{D}_{\mathfrak{a}, G}^{p, 1}$ consists of those $F \in L^{p}\left(C_{0} \mathbf{R}^{m} ; G\right)$ s.t.

1. $F$ is a-measurable,

2. $F \in \operatorname{Dom}_{G}\left(d^{p}\right)$.

Inductively $\mathbb{D}_{\mathfrak{a}, G}^{p, k}$ consists of those $F \in L^{p}\left(C_{0} \mathbf{R}^{m} ; G\right)$ such that

1. $F \in \mathbb{D}_{\mathfrak{a}, G}^{p, 1}$ 
2. $\mathbf{E}\left\{d^{p} F \mid \mathfrak{a}\right\}: C_{0} R^{m} \rightarrow L_{2}(H ; G)$ is in $\mathbb{D}_{\mathfrak{a}, \mathbb{L}_{2}(H ; G)}^{p, k-1}$.

For $F \in \mathbb{D}_{\mathfrak{a}, G}^{p, k}$ define

$$
\left(\|F\|_{\mathbb{D}_{\mathfrak{a}, G}^{p, k}}\right)=\left(\sum_{r=0}^{k}\left\|\left(d^{p} \mathbf{E}\{-\mid \mathfrak{a}\}\right)^{r} F\right\|_{L^{p}}^{p}\right)^{\frac{1}{p}}
$$

These spaces are Banach spaces since $d^{p} \mathbf{E}\{-\mid \mathfrak{a}\} F$ is the composition of a closed operator following a bounded operator and thus a closed operator.

The following theorem corrects the version in [25].

Theorem 8.14 Assume Condition $(M)$. Let $G$ be a separable Hilbert space. Then $f: \mathcal{C}_{x_{0}} M \rightarrow G$ is in $W^{p, k}\left(\mathcal{C}_{x_{0}} M ; G\right)$ some $1 \leq p<\infty, k \in\{1,2, \ldots\}$ if and only if $\mathcal{I}^{*}(f) \in \mathbb{D}_{\mathcal{F}, G}^{p, k}$. Moreover

$$
\mathcal{I}^{*}: W^{p, k}\left(\mathcal{C}_{x_{0}} M ; G\right) \rightarrow \mathbb{D}_{\mathcal{F}, G}^{p, k}
$$

is a continuous linear isomorphism.

Proof For $k=1$ this is Proposition 8.1. Suppose it holds for some $k \in\{1,2, \ldots\}$. Then if $f \in W^{p, 1}\left(\mathcal{C}_{x_{0}} M ; G\right)$

$$
\mathbf{E}\left\{d \mathcal{I}^{*}(f) \mid \mathcal{F}^{x_{0}}\right\}=\mathbf{E}\left\{\mathcal{I}^{*}(\tilde{d} f) \mid \mathcal{F}^{x_{0}}\right\}=\mathcal{I}^{*}(\tilde{d} f \circ \overline{T \mathcal{I}})=\mathcal{I}^{*}(\tilde{d} f \circ \boldsymbol{X}),
$$

which belongs to $\mathbb{D}^{p, k}$ if and only if $\tilde{d} f \circ \boldsymbol{X} \in W^{p, k}\left(\mathcal{C}_{x_{0}} M ; H^{*}\right)$. The last holds if and only if $f \in W^{p, k+1}\left(\mathcal{C}_{x_{0}} M ; G\right)$ by Proposition 8.11 and so the result holds for $k+1$.

Corollary 8.15 Under the conditions of Theorem $8.14, \mathcal{I}^{*}$ maps $\mathbb{D}^{p, k}\left(\mathcal{C}_{x_{0}} M ; G\right)$ onto a closed subspace of $\mathbb{D}_{\mathcal{F}, G}^{p, k}$.

Note that many authors, e.g. Léandre [33], Li [34], have defined Sobolev spaces for $\mathcal{C}_{x_{0}} M$ using the flat connection on $\mathcal{H}$ defined by the trivialisation of $\mathcal{H}$ given by $V . \mapsto$ $/ /{ }^{-1} V$. (in the Levi-Civita case). This cannot be expected to agree with our definition because of the curvature term occurring in the covariant derivative of $/ /-1$, c.f. the proof of Lemma 6.11. In particular Corollary 8.15 should not in general hold with those definitions. For a covariant calculus using Markovian connections see CruzeiroMalliavin [7].

\section{Special case: no redundant noise}

Suppose $X(x): \mathbf{R}^{m} \rightarrow T_{x} M$ is injective for each $x$, so $p=m$ and $X$ trivialises $E$. Or equivalently $\mathcal{F}=\mathcal{F}^{x_{0}}$. Examples include left and right invariant stochastic differential equations on Lie groups, Example 2 of section 2.1.1 and the canonical stochastic differential equation on the orthonormal frame bundle, Example 4 of the same section. Condition $(M)$ may not hold for the injective case, see for example $2 \mathrm{C}$ on page 24 of Elworthy-LeJan-Li [15]. However we still get complete intertwining and all the results in this article. In fact condition $\left(M_{0}\right)$ can be removed in the proof of the key Theorems, Th 3.4, Th 4.2, Co 4.3 and their corresponding Hilbert space 
valued versions. Furthermore the Conditions $K$ needed to define $\mathbb{D}^{p, k}$ holds trivially. The following theorem extends some of the results of Fang-Franchi [27] who were concerned with Brownian motion measure on Lie groups.

Theorem 9.1 Suppose $X$ is injective. Then for $1<p<\infty$

1. $\mathcal{I}^{*}$ maps $\mathbb{D}^{p, k}\left(\mathcal{C}_{x_{0}} M ; \mathbf{R}\right)$ isometrically onto $\mathbb{D}^{p, k}(\Omega ; \mathbf{R}), k=1,2, \ldots$, and

$$
\begin{aligned}
\mathcal{I}^{*} d^{p} & =d^{p} \mathcal{I}^{*} \\
\mathcal{I}^{*} \mathbb{W}^{p,(k)} d^{p} & =\left(d^{p}\right)^{(k+1)} \mathcal{I}^{*}
\end{aligned}
$$

where, for $\psi \in L^{p} \Gamma \mathbb{L}\left(\otimes^{k+1} \mathcal{H} ; \mathbf{R}\right)$,

$$
\mathcal{I}^{*}(\psi): \Omega \rightarrow \mathbb{L}\left(\otimes^{k+1} \mathcal{H} ; \mathbf{R}\right)
$$

is given by $\mathcal{I}^{*}(\psi)=\left(\psi \circ \otimes^{(k+1)} \overline{T \mathcal{I}}\right) \circ \mathcal{I}$.

2. On $\mathcal{H}$-1-forms $\left(d^{p}\right)^{*} \mathcal{I}^{*}=\mathcal{I}^{*}\left(d^{p}\right)^{*}$. An $\mathcal{H}$-vector field $V$ lies in $\operatorname{Dom}\left(\operatorname{div}_{\mathcal{C}_{x_{0}} M}^{p}\right)$ if and only if $(\mathbb{Y} V) \circ \mathcal{I}$ is in $\operatorname{Dom}\left(\operatorname{div}_{\Omega}^{p}\right)$; if so

$$
(\operatorname{div} V) \circ \mathcal{I}=-\int_{0}^{T}\left\langle\frac{\mathbb{D}}{d s} V\left(x_{s}\right), X\left(x_{s}\right) d B_{s}\right\rangle_{x_{s}}
$$

where the integral is a Skorohod integral.

3. The Laplacians, or 'Ornstein-Uhlenbeck operators', are conjugated by $\mathcal{I}^{*}$, as operators on $L^{p}$ :

$$
\mathcal{I}^{*}\left(\left(d^{p}\right)^{*} d^{p}=\left(d^{p}\right)^{*} d^{p} \mathcal{I}^{*}\right.
$$

Proof Since $T \mathcal{I}=\overline{T \mathcal{I}}$, it acts isometrically on $H$ with inverse $\mathbb{Y}$. The proof of Proposition 5.1 gives that $h \in \mathcal{D}$ om $\left(\operatorname{div}_{\Omega}^{p}\right)$ if and only if $T \mathcal{I}(h) \in \operatorname{Dom}\left(\operatorname{div}^{p}\right)$. Part 2 follows by (5.4). For $k=1$, part 1 follows from part 2 since, by (3.8), $\mathcal{I}^{*}$ acts isometrically on $\mathcal{H}$-1-forms as well as on functions. For $k>1$ and higher order Sobolev spaces, observe that for $G$ a separable Hilbert space, $\mathbb{D}_{\mathcal{F}^{x_{0} ; G}}^{p, k}=\mathbb{D}^{p, k}(\Omega, G)$. Finally part 3 follows from part 1 and part 2 .

Remark 9.2 From Theorem 9.1 we see Markov uniqueness holds if $X$ is injective, without assuming condition $\left(M_{0}\right)$. In fact the proof in section 6.3 shows that $\mathrm{Cyl}^{0} \mathcal{H}^{*}$ is total in $\operatorname{Dom}\left(d^{*}\right)$ on $\mathcal{C}_{x_{0}} M$ and so $\mathbb{D}^{2,1} \mathcal{H}^{*}$ is dense in $\operatorname{Dom}\left(d^{*}\right)$ and $\overline{\left.\left(d^{p}\right)^{*}\right|_{\mathrm{Cyl}^{0} \mathcal{H}^{*}}}=$

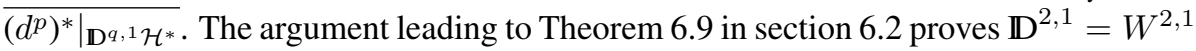
and Markov uniqueness. For the stronger result of the essential self-adjointness of $\left.d^{*} d\right|_{\text {Cyl }}$ on $L^{2}\left(\mathcal{C}_{x_{0}} M ; \mathbf{R}\right)$ see the method of Aida [3].

For completeness and as an example we show that for Lie groups $G$ with left invariant connection our connection $\mathbb{\nabla}$ agrees with the 'Levi-Civita' connection used previously, c.f. Freed [28], Fang-Franchi [27], Aida [4], Driver-Lohrenz [10]. See also Shigekawa [43], Shigekawa-Taniguchi [44]. For this let $M=G$ with left invariant stochastic differential equation as in Example 2 of section 2.1.1. Since the adjoint connection $\hat{\nabla}$ of $\nabla$ is the flat right invariant connection we have $\mathcal{H}$-vector fields $V^{y}(\sigma)$ given by $V(\sigma)_{t}:=T R_{\sigma(t)}\left(y_{t}\right), y \in L_{0}^{2,1}([0, T] ; \mathfrak{g})$, for $T R_{g}: \mathfrak{g}=T_{e} G \rightarrow T_{g} M$ the derivative of right translation $R_{g}$ by $g \in G$. 
Proposition 9.3 For $y, z$ in $L_{0}^{2,1}([0, T] ; \mathfrak{g})$,

$$
\left(\mathbb{\nabla}_{V^{z}(\sigma)} V^{y}\right)_{t}=T R_{\sigma(t)}[\dot{y}(t), z(t)] .
$$

Proof By definition of $X$ and $\mathbb{V}$ and since the Ricci curvature is zero and there is no 'drift',

$$
\begin{aligned}
\left(\mathbb{\nabla}_{V^{z}(\sigma)} V^{y}\right)_{t} & =T L_{\sigma(t)}\left[d\left(\rho \mapsto T L_{\rho}^{-1} \frac{\mathbb{D}}{d \cdot} V^{y}\right)_{\sigma}\left(V^{z}(\sigma)\right)\right]_{t} \\
& =T L_{\sigma(t)}\left[d\left(g \mapsto T L_{g}^{-1} T R_{g} \dot{y}_{t}\right)_{\sigma(t)}\left(T R_{\sigma(t)}\left(z_{t}\right)\right)\right] \\
& =T R_{\sigma(t)}[\dot{y}(t), z(t)]
\end{aligned}
$$

as expected.

\section{Appendix: The conditional expectations of exponential martin- gales}

Let $\varepsilon(a)$ be the exponential martingale

$$
\varepsilon(a):=\exp \left(\int_{0}^{T}\left\langle\dot{a}_{s}, d B_{s}\right\rangle-\frac{1}{2} \int_{0}^{T}\left|\dot{a}_{s}\right|^{2} d s\right)
$$

for $a \in H$ and set $\tilde{\varepsilon}(a)=\mathbf{E}\left\{\varepsilon(a) \mid \mathcal{F}^{x_{0}}\right\}$. By Proposition 7.3 we know $\tilde{\varepsilon}(a)$ is in $\mathbb{D}_{\mathcal{F}^{x_{0}}}^{2,1}$. As evidence that $\mathbf{E}\left\{-\mid \mathcal{F}^{x_{0}}\right\}$ maps $\mathbb{D}^{2,1}$ into itself we show, c.f. Remark 7.9:

Proposition 10.1 There is a constant $C$ such that for all $a \in H$

$$
\|\tilde{\varepsilon}(a)\|_{\mathbb{D}^{2,1}} \leq C\|\varepsilon(a)\|_{\mathbb{D}^{2,1}} .
$$

Proof Set

$$
\varepsilon_{t}=\varepsilon_{t}(a)=\exp \left(\int_{0}^{t}\left\langle\dot{a}_{s}, d B_{s}\right\rangle-\frac{1}{2} \int_{0}^{t}\left|\dot{a}_{s}\right|^{2} d s\right) .
$$

Denote by $d^{H}$ the $H$-derivative to distinguish it from the stochastic differential. Then $\mathbf{E}\left|\varepsilon_{t}\right|^{2}=\exp \left(\int_{0}^{t}\left|\dot{a}_{s}\right|^{2} d s\right)$ and $\|\varepsilon(a)\|_{\mathbb{D}^{2,1}}^{2}=\left(1+\|a\|_{H}^{2}\right) \exp \left(\|a\|_{H}^{2}\right)$. Conditioning the stochastic differential equation

$$
d \varepsilon_{t}=\left\langle\dot{a}_{t}, d B_{t}\right\rangle \varepsilon_{t}
$$

on $\mathcal{F}^{x_{0}}$ shows that $\tilde{\varepsilon}_{t} \equiv \tilde{\varepsilon}_{t}(a)=\mathbf{E}\left\{\varepsilon_{t} \mid \mathcal{F}^{x_{0}}\right\}$ satisfies:

$$
d \tilde{\varepsilon}_{t}=\tilde{\varepsilon}_{t}\left\langle X\left(x_{t}\right) \dot{a}_{t}, X\left(x_{t}\right) d B_{t}\right\rangle .
$$

So

$$
\tilde{\varepsilon}_{t}=\exp \left(\int_{0}^{t}\left\langle X\left(x_{s}\right) \dot{a}_{s}, X\left(x_{s}\right) d B_{s}\right\rangle-\frac{1}{2} \int_{0}^{t}\left|X\left(x_{s}\right) \dot{a}_{s}\right|^{2} d s\right)
$$

and

$$
\mathbf{E}\left|\bar{\varepsilon}_{t}\right|^{2}=\mathbf{E} \exp \left(\int_{0}^{t}\left|K^{\perp}\left(x_{s}\right) \dot{a}_{s}\right|^{2} d s\right)
$$


where $K^{\perp}: M \times \mathbf{R}^{m} \rightarrow[\operatorname{ker}(X)]^{\perp}$ is the projection map $Y(x) X(x)$. From this, for $h \in H$,

$$
\begin{aligned}
d^{H}(\tilde{\varepsilon})(h)=\tilde{\varepsilon} & \left\{\int_{0}^{T}\left\langle X\left(x_{s}\right) \dot{a}_{s}, X\left(x_{s}\right)\left(\dot{h}_{s}\right) d s+\nabla_{T \mathcal{I}_{s}(h)} X d B_{s}\right\rangle\right. \\
& \left.+\int_{0}^{T}\left\langle\nabla_{T \mathcal{I}_{s}(h)} X\left(\dot{a}_{s}\right), X\left(x_{s}\right) d B_{s}\right\rangle-\int_{0}^{T}\left\langle\nabla_{T \mathcal{I}_{s}(h)} X\left(x_{s}\right)\left(\dot{a}_{s}\right), X\left(x_{s}\right) \dot{a}_{s}\right\rangle d s\right\} .
\end{aligned}
$$

By Theorem 3.4, setting $\phi^{\#}=\tilde{\varepsilon} \mathbf{W}_{s}(X(x.) \dot{a}$.$) , then$

$$
\tilde{\varepsilon} \int_{0}^{T}\left\langle X\left(x_{s}\right) \dot{a}_{s}, X\left(x_{s}\right)\left(\dot{h}_{s} d s\right)+\nabla X\left(T \mathcal{I}_{s}(h)\right) d B_{s}\right\rangle=\mathcal{I}^{*}(\phi)(h) .
$$

Note that $\|\phi\|^{2}=\mathbf{E} \tilde{\varepsilon}^{2} \int_{0}^{T}\left|X\left(x_{s}\right) \dot{a}_{s}\right|^{2} d s \leq|a|_{H}^{2} \mathbf{E} \tilde{\varepsilon}^{2}$.

Take any Riemannian metric on $T M$ extending that of $E$ and for $e \in \mathbf{R}^{m}$ define:

$$
Z_{x}(e): E_{x} \rightarrow T_{x} M
$$

to be the adjoint of $\nabla_{-} X(e): T_{x} M \rightarrow E_{x}$ for each $x$ in $M$. Then

$$
\begin{aligned}
& \tilde{\varepsilon}\left\{\int_{0}^{T}\left\langle\nabla_{T \mathcal{I}_{s}(h)} X\left(\dot{a}_{s}\right), X\left(x_{s}\right) d B_{s}\right\rangle-\int_{0}^{T}\left\langle\nabla_{T \mathcal{I}_{s}(h)} X\left(x_{s}\right)\left(\dot{a}_{s}\right), X\left(x_{s}\right) \dot{a}_{s}\right\rangle d s\right\} \\
= & \left.\tilde{\varepsilon}\left\{\int_{0}^{T}\left\langle T \mathcal{I}_{s}(h), Z_{x_{s}}\left(\dot{a}_{s}\right)\left(X\left(x_{s}\right) d B_{s}\right)\right\rangle-\int_{0}^{T}\left\langle T \mathcal{I}_{s}(h), Z_{x_{s}}\left(\dot{a}_{s}\right)\left(X\left(x_{s}\right) \dot{a}_{s}\right)\right)\right\rangle d s\right\},
\end{aligned}
$$

which can be verified to equal to $\mathcal{I}^{*}(\psi)(h)$, defined by Theorem 3.4, where

$$
\psi^{\#}=\tilde{\varepsilon} \mathbf{W}_{t}\left(\Pi\left(W_{.}^{-1}\right)^{*} \int_{.}^{T} W_{r}^{*} Z_{\sigma_{r}}\left(\dot{a}_{r}\right) X\left(\sigma_{r}\right)\left(d B_{r}-\dot{a}_{r} d r\right)\right) .
$$

We have used the expression (3.12) of $T \mathcal{I}$ for the verification. It remains to estimate

$$
\begin{aligned}
\mathbf{E}\left|\mathcal{I}^{*}(\psi)\right|^{2} & =\int_{C_{x_{0}} M} \int_{0}^{T}\left|\frac{\mathbb{D}}{d s} \psi_{s}^{\#}\right|^{2} d s \mu_{x_{0}}(d \sigma) \\
& =\mathbf{E} \tilde{\varepsilon}^{2} \int_{0}^{T}\left(\Pi\left(W_{t}^{-1}\right)^{*} \int_{t}^{T} W_{r}^{*} Z_{x_{r}}\left(\dot{a}_{r}\right) X\left(x_{r}\right)\left(d B_{r}-\dot{a}_{r} d r\right)\right)^{2} d t .
\end{aligned}
$$

For this let $\left\{y_{t}: 0 \leq t \leq T\right\}$ be the solution to the stochastic differential equation

$$
\left\{\begin{array}{l}
d y_{t}=X\left(y_{t}\right) \circ d B_{t}+A\left(y_{t}\right) d t+2 X\left(y_{t}\right)\left(\dot{a}_{t}\right) d t \\
y_{0}=x_{0} .
\end{array}\right.
$$

Then by the Girsanov-Maruyama theorem,

$$
\begin{aligned}
& \mathbf{E}\left|\mathcal{I}^{*}(\psi)\right|^{2} \\
= & \mathbf{E} \exp \left(\int_{0}^{T}\left|K^{\perp}\left(y_{s}\right) \dot{a}_{s}\right|^{2} d s\right) \int_{0}^{T}\left(\Pi\left(W_{t}^{-1}\right)^{*} \int_{t}^{T} W_{r}^{*} Z_{y_{r}}\left(\dot{a}_{r}\right) X\left(y_{r}\right)\left(d B_{r}+\dot{a}_{r} d r\right)\right)^{2} d t
\end{aligned}
$$




$$
\begin{gathered}
\leq \quad 2 \mathbf{E} \exp \left(\int_{0}^{T}\left|K^{\perp}\left(y_{s}\right) \dot{a}_{s}\right|^{2} d s\right) \int_{0}^{T}\left(\Pi\left(W_{t}^{-1}\right)^{*} \int_{t}^{T} W_{r}^{*} Z_{y_{r}}\left(\dot{a}_{r}\right)\left(X\left(y_{r}\right) d B_{r}\right)\right)^{2} d t \\
+2 \mathbf{E} \exp \left(\int_{0}^{T}\left|K^{\perp}\left(y_{s}\right) \dot{a}_{s}\right|^{2} d s\right) \int_{0}^{T}\left(\Pi\left(W_{t}^{-1}\right)^{*} \int_{t}^{T} W_{r}^{*} Z_{y_{r}}\left(\dot{a}_{r}\right)\left(X\left(y_{r}\right) \dot{a}_{r}\right) d r\right)^{2} d t \\
\leq \quad \text { const. }\|a\|_{H}^{2} \exp \left(\|a\|_{H}^{2}\right) \\
+\exp \left(\|a\|_{H}^{2}\right) \mathbf{E} \exp \left(-\int_{0}^{T}\left|K\left(y_{s}\right) \dot{a}_{s}\right|^{2} d s\right) . \\
\int_{0}^{T}\left(\Pi\left(W_{t}^{-1}\right)^{*} \int_{t}^{T} W_{r}^{*} Z_{y_{r}}\left(K\left(y_{r}\right) \dot{a}_{r}\right) X\left(y_{r}\right)\left(\dot{a}_{r}\right) d r\right)^{2} d t
\end{gathered}
$$

where $K(x)=1-K^{\perp}(x)$, using the fact that $\nabla X=\nabla X \circ K$. Now

$$
\begin{aligned}
& \mathbf{E} \exp \left(-\int_{0}^{T}\left|K\left(y_{s}\right) \dot{a}_{s}\right|^{2} d s\right) \cdot \int_{0}^{T}\left(\Pi\left(W_{t}^{-1}\right)^{*} \int_{t}^{T} W_{r}^{*} Z_{y_{r}}\left(K\left(y_{r}\right) \dot{a}_{r}\right) X\left(y_{r}\right)\left(\dot{a}_{r}\right) d r\right)^{2} d t \\
\leq & \text { const.E } \exp \left(-\int_{0}^{\mathrm{T}}\left|\mathrm{K}\left(\mathrm{y}_{\mathrm{s}}\right) \dot{\mathrm{a}}_{\mathrm{s}}\right|^{2} \mathrm{ds}\right) \cdot \int_{0}^{\mathrm{T}}\left\{\int_{\mathrm{t}}^{\mathrm{T}}\left|\mathrm{K}\left(\mathrm{y}_{\mathrm{r}}\right) \dot{\mathrm{a}}_{\mathrm{r}}\right|^{2} \mathrm{dr} \int_{\mathrm{t}}^{\mathrm{T}}\left|\mathrm{K}^{\perp}\left(\mathrm{y}_{\mathrm{r}}\right) \dot{\mathrm{a}}_{\mathrm{r}}\right|^{2} \mathrm{dr}\right\} \mathrm{dt} \\
\leq & \text { const. }|\mathrm{a}|_{\mathrm{H}}^{2} \mathbf{E} \int_{0}^{\mathrm{T}}\left(\int_{\mathrm{t}}^{\mathrm{T}} \exp \left(-\int_{\mathrm{r}}^{\mathrm{T}}\left|\mathrm{K}\left(\mathrm{y}_{\mathrm{s}}\right) \dot{\mathrm{a}}_{\mathrm{s}} \mathrm{d}_{\mathrm{s}}\right|^{2} \mathrm{ds}\right)\left|\mathrm{K}^{\perp}\left(\mathrm{y}_{\mathrm{r}}\right) \dot{\mathrm{a}}_{\mathrm{r}}\right|^{2} \mathrm{dr}\right) \mathrm{dt} \\
\leq & \text { const. }|\mathrm{a}|_{\mathrm{H}}^{2} \mathbf{E} \int_{0}^{\mathrm{T}} \exp \left(-\int_{\mathrm{t}}^{\mathrm{T}}\left|\mathrm{K}\left(\mathrm{y}_{\mathrm{s}}\right) \dot{\mathrm{a}}_{\mathrm{s}} \mathrm{d}_{\mathrm{s}}\right|^{2} \mathrm{ds}\right) \mathrm{dt} \\
\leq & \text { const. }|\mathrm{a}|_{\mathrm{H}}^{2} .
\end{aligned}
$$

From this we see $\mathbf{E}\left|\mathcal{I}^{*}(\psi)\right|^{2} \leq$ const. $|\mathrm{a}|_{\mathrm{H}}^{2} \mathrm{e}^{|\mathrm{a}|_{\mathrm{H}}^{2}}$ as required.

\section{Acknowledgement}

This research was partially supported by EPSRC research grant GR/H67263 and NSF research grant DMS 0072387. Xue-Mei Li would like to acknowledge the support from the Royal Society and the Leverhulme Trust for her Senior Research Fellowship. The research benefited from our contacts with many colleagues, especially S. Aida, S. Albeverio, A. Eberle, S. Fang, Y. LeJan, Z. Ma, P. Malliavin, and M. Röckner.

\section{Addresses:}

K.D. Elworthy, Mathematics Institute, University of Warwick, Coventry CV4 7AL, U.K. Xue-Mei Li (Xue-Mei Hairer), Mathematical Sciences, Loughborough University, Loughborough, LE11 3TU U.K. Email: xue-mei.li@lboro.ac.uk.

\section{References}

[1] S. Aida. On the irreducibility of certain Dirichlet forms on loop spaces over compact homogeneous spaces. In K. D. Elworthy, S. Kusuoka, and I. Shigekawa, editors, New Trends in stochastic Analysis, Proc. Taniguchi Symposium, Sept. 1995, Charingworth, pages 3-42. World Scientific Press, 1997. 
[2] S. Aida and K.D. Elworthy. Differential calculus on path and loop spaces. 1. Logarithmic Sobolev inequalities on path spaces. C. R. Acad. Sci. Paris, t. 321, série I, pages 97-102, 1995.

[3] Shigeki Aida. On the Ornstein-Uhlenbeck operators on Wiener-Riemannian manifolds. $J$. Funct. Anal., 116(1):83-110, 1993.

[4] Shigeki Aida. Sobolev spaces over loop groups. J. Funct. Anal., 127(1):155-172, 1995.

[5] A. B. Cruzeiro and S. Fang. Une inégalité $l^{2}$ pour des intégrales stochastiques anticipatives sur une variété riemannienne. C. R. Acad. Sci. Paris, Série I, 321:1245-1250, 1995.

[6] A. B. Cruzeiro and P. Malliavin. Renormalized differrential geometry on path spaces: Structural equation, curvature. J. Funct. Anal., 139:119-181, 1996.

[7] A. B. Cruzeiro and P. Malliavin. Frame bundle of Riemannian path space and Ricci tensor in adapted differential geometry. J. Funct. Anal., 177:219-253, 2000.

[8] Giuseppe Da Prato and Jerzy Zabczyk. Second order partial differential equations in Hilbert spaces, volume 293 of London Mathematical Society Lecture Note Series. Cambridge University Press, Cambridge, 2002.

[9] B. K. Driver. A Cameron-Martin type quasi-invariance theorem for Brownian motion on a compact Riemannian manifold. J. Functional Analysis, 100:272-377, 1992.

[10] B. K. Driver and T. Lohrenz. Logarithmic Sobolev inequalities for pinned loop groups. $J$. Funct. Anal., 140(2):381-448, 1996.

[11] Bruce K. Driver. The non-equivalence of Dirichlet forms on path spaces. In Stochastic analysis on infinite-dimensional spaces (Baton Rouge, LA, 1994), pages 75-87. Longman Sci. Tech., Harlow, 1994.

[12] Andreas Eberle. Uniqueness and non-uniqueness of semigroups generated by singular diffusion operators. Lecture Notes in Mathematics, 1718. Springer-Verlag, 1999.

[13] J. Eells. On the geometry of function spaces. In Symposium internacional de topología algebraica International symposium on algebraic topology, pages 303-308. Universidad Nacional Autónoma de México and UNESCO, Mexico City, 1958.

[14] H. Eliasson. Geometry of manifolds of maps. J. Diff. Geom., 1:169-194, 1967.

[15] K. D. Elworthy, Y. LeJan, and X.-M. Li. On the geometry of diffusion operators and stochastic flows, Lecture Notes in Mathematics 1720. Springer, 1999.

[16] K. D. Elworthy, Y. LeJan, and Xue-Mei Li. Integration by parts formulae for degenerate diffusion measures on path spaces and diffeomorphism groups. C.R. Acad. Sci. Paris, t. 323 serié 1:921-926, 1996.

[17] K. D. Elworthy, Y. LeJan, and Xue-Mei Li. Concerning the geometry of stochastic differential equations and stochastic flows. In K.D. Elworthy, S. Kusuoka, and I. Shigekawa, editors, New Trends in stochastic Analysis, Proc. Taniguchi Symposium, Sept. 1995, Charingworth. World Scientific Press, 1997.

[18] K. D. Elworthy and Xue-Mei Li. A class of integration by parts formulae in stochastic analysis I. In Itô's Stochastic Calculus and Probability Theory (dedicated to Kiyosi Itô on the occasion of his eightieth birthday). Springer, 1996.

[19] K. D. Elworthy and Xue-Mei Li. Special Itô maps and an $L^{2}$ Hodge theory for one forms on path spaces. In Stochastic processes, physics and geometry: new interplays, I (Leipzig, 1999), pages 145-162. Amer. Math. Soc., 2000.

[20] K. D. Elworthy and Xue-Mei Li. Some families of $q$-vector fields on path spaces. Infin. Dimens. Anal. Quantum Probab. Relat. Top., 6(suppl.):1-27, 2003.

[21] K. D. Elworthy and Xue-Mei Li. Intertwining and the Markov uniqueness problem on path spaces. To appear in: Stochastic Partial Differential Equations and Applications VII, Lecture Notes in Pure and Applied Mathematics, 2004. 
[22] K. D. Elworthy and Zhi-Ming Ma. Admissible vector fields and related diffusions on finitedimensional manifolds. Ukraïn. Mat. Zh., 49(3):410-423, 1997.

[23] K. D. Elworthy and M. Yor. Conditional expectations for derivatives of certain stochastic flows. In J. Azéma, P.A. Meyer, and M. Yor, editors, Sem. de Prob. XXVII. Lecture Notes in Mathematics 1557, pages 159-172. Springer-Verlag, 1993.

[24] K. David Elworthy and Xue-Mei Li. Bismut type formulae for differential forms. C. R. Acad. Sci., Sér/ I, Math. Paris, 327(1):87-92, 1998.

[25] K. David Elworthy and Xue-Mei Li. Gross-Sobolev spaces on path manifolds: uniqueness and intertwining by Itô maps. C. R. Math. Acad. Sci. Paris, 337(11):741-744, 2003.

[26] K. David Elworthy and Zhi-Ming Ma. Vector fields on mapping spaces and related Dirichlet forms and diffusions. Osaka J. Math., 34(3):629-651, 1997.

[27] S. Fang and J. Franchi. A differentiable isomorphism between Wiener space and path group. In Séminaire de Probabilités, XXXI, volume 1655 of Lecture Notes in Math., pages 54-61. Springer, Berlin, 1997.

[28] Daniel S. Freed. The geometry of loop groups. J. Differential Geom., 28(2):223-276, 1988.

[29] Elton P. Hsu. Quasi-invariance of the Wiener measure on the path space over a compact Riemannian manifold. J. Funct. Anal., 134(2):417-450, 1995.

[30] N. Ikeda and S. Watanabe. Stochastic Differential Equations and Diffusion Processes, second edition. North-Holland, 1989.

[31] J. D. S. Jones and R. Léandre. $L^{p}$-Chen forms on loop spaces. In Stochastic analysis (Durham, 1990), volume 167 of London Math. Soc. Lecture Note Ser., pages 103-162. Cambridge Univ. Press, Cambridge, 1991.

[32] M. Krée and P. Krée. Continuité de la divergence dans les espaces de sobolev relatifs l'espace de wiener. C. R. Acad. Sci. Paris Sèr. I Math, 296(20):833-836, (1983).

[33] R. Léandre. Integration by parts formulas and rotationally invariant Sobolev calculus on free loop spaces. J. Geom. Phys., 11(1-4):517-528, 1993. Infinite-dimensional geometry in physics (Karpacz, 1992).

[34] Xiang-Dong Li. Sobolev spaces and capacities theory on path spaces over a compact Riemannian manifold. Probab. Theory Relat. Fields, 125:96-134, 2003.

[35] P. Malliavin. Stochastic analysis, Grundlehren der Mathematischen Wissenschaften, 313. Springer-Verlag, 1997.

[36] Paul Malliavin. Stochastic calculus of variation and hypoelliptic operators. In Proceedings of the International Symposium on Stochastic Differential Equations (Res. Inst. Math. Sci., Kyoto Univ., Kyoto, 1976), pages 195-263, New York, 1978. Wiley.

[37] M. S. Narasimhan and S. Ramanan. Existence of universal connections. Amer. J. Math., 83:563-572, 1961

[38] D. Nualart. The Malliavin Calculus and Related Topics. Springer-Verlag, 1995.

[39] É. Pardoux and S. G. Peng. Adapted solution of a backward stochastic differential equation. Systems Control Lett., 14(1):55-61, 1990.

[40] D. Quillen. Superconnections; character forms and the Cayley transform. Topology, 27(2):211-238, 1988.

[41] I. Shigekawa. A quasihomeomorphism on the Wiener space. Proc. Sympos. Pure math. , American Math. Soc, 57:473-486, 1995.

[42] Ichirō Shigekawa. de Rham-Hodge-Kodaira's decomposition on an abstract Wiener space. J. Math. Kyoto Univ., 26(2):191-202, 1986. 
[43] Ichiro Shigekawa. Differential calculus on a based loop group. In New trends in stochastic analysis (Charingworth, 1994), pages 375-398. World Sci. Publishing, River Edge, NJ, 1997.

[44] Ichiro Shigekawa and Setsuo Taniguchi. A Kähler metric on a based loop group and a covariant differentiation. In Itô's stochastic calculus and probability theory, pages 327 346. Springer, Tokyo, 1996.

[45] H. Sugita. On a characterization of the Sobolev spaces over an abstract wiener space. $J$. Math. Kyoto Univ., 25(4):717-725, 1985.

[46] M. Takeda. On the uniqueness of Markovian self-adjoint extension of diffusion operators on infinite dimensional spaces. Osaka J. Math., 22(4):733-742, 1985.

[47] Kôsaku Yosida. Functional analysis. Die Grundlehren der Mathematischen Wissenschaften, Band 123. Academic Press Inc., New York, 1965. 